\title{
WestVirginiaUniversity
}

THE RESEARCH REPOSITORY @ WVU

Graduate Theses, Dissertations, and Problem Reports

2012

\section{Decentralized Synergetic Control of Power Systems}

\author{
Taoridi Ademoye \\ West Virginia University
}

Follow this and additional works at: https://researchrepository.wvu.edu/etd

\section{Recommended Citation}

Ademoye, Taoridi, "Decentralized Synergetic Control of Power Systems" (2012). Graduate Theses, Dissertations, and Problem Reports. 155.

https://researchrepository.wvu.edu/etd/155

This Dissertation is protected by copyright and/or related rights. It has been brought to you by the The Research Repository @ WVU with permission from the rights-holder(s). You are free to use this Dissertation in any way that is permitted by the copyright and related rights legislation that applies to your use. For other uses you must obtain permission from the rights-holder(s) directly, unless additional rights are indicated by a Creative Commons license in the record and/ or on the work itself. This Dissertation has been accepted for inclusion in WVU Graduate Theses, Dissertations, and Problem Reports collection by an authorized administrator of The Research Repository @ WVU.

For more information, please contact researchrepository@mail.wvu.edu. 


\title{
Decentralized Synergetic Control of Power Systems
}

\author{
by \\ Taoridi Ademoye \\ Dissertation submitted to the \\ College of Engineering and Mineral Resources \\ at West Virginia University \\ in partial fulfillment of the requirements \\ for the degree of
}

Doctor of Philosophy

in

Electrical Engineering

Prof. Hong-Jian Lai, Ph.D.

Professor Asadollah Davari

Prof. Muhammad A. Choudhry, Ph.D.

Prof. Powsiri Klinkhachorn, Ph.D.

Prof. Ali Feliachi, Ph.D., Chair

Lane Department of Computer Science and Electrical Engineering

Morgantown, West Virginia

2012

Keywords: Synergetic Control, Particle Swarm Optimization, Reinforcement Learning, Power Analysis Toolbox 


\author{
Abstract \\ Decentralized Synergetic Control of Power Systems \\ by \\ Taoridi Ademoye \\ Doctor of Philosophy in Electrical Engineering \\ West Virginia University \\ Professor Ali Feliachi, Ph. D., Chair
}

The objective of this dissertation is to design decentralized controllers to enhance the transient stability of power systems. Due to the nonlinearities and complexities of the system, nonlinear control design techniques are required to improve its dynamic performance. In this dissertation a synergetic control technique is being proposed to design supplementary controller that is added to the exciter of the generation unit of the system. Although this method has been previously applied to a Single Infinite Machine Bus (SMIB) system with high degree of success, it has not been employed to systems with multi machine. Also, the method has good robust characteristic like that of the popular Sliding Mode Control (SMC) technique. But the latter technique introduces steady state chattering effect which can cause wear and tear in actuating system. This gives the proposed technique a major advantage over the SMC. In this work, the method is employed for systems with multi machine. Each of the machines is considered to be a subsystem and decentralized controller is designed for each subsystem. The interconnection term of each subsystem with the rest of the system is estimated by a polynomial function of the active power generated by the subsystem. Particle Swarm Optimization (PSO) technique is employed for optimum tuning of the controller's parameters. To further enhance the performance of the system by widening its range of operation, Reinforcement Learning (RL) technique is used to vary the gains of the decentralized synergetic supplementary controller in real time. The approach is illustrated with several case studies including a SMIB system with or without a Static Var Compensator (SVC), a Two Area System (TAS) with or without an SVC, a three -machines-nine-bus system and a fifty machine system. Results show that the proposed control technique provides better damping than the conventional power system stabilizers and synergetic controllers with fixed gains. 


\section{Acknowledgements}

I bless God Almighty for His faithfulness in my life, and for the strength, sound health and sound mind He granted me throughout the period of the program. I also thank Him for blessing me with a lovely wife and a wonderful son during this period.

I am very grateful to my supervisor and mentor Dr Ali Feliachi for his advice, support and guidance over the years. He has impacted into my life an immense deal of knowledge and without him, this work would not have been accomplished.

I also appreciate all the faculty members who have taught, encouraged and guided me in special and different ways. Special thanks go to Professor Muhammad Choudhry (WVU Lane Department of Computer Science and Electrical Engineering), Professor Asadollah Davari (WVU Tech. Department of Electrical and Computer Engineering), Professor Powsiri Klinkhachorn (Lane department of Computer Science and Electrical Engineering), and Professor Hong- Jian Lai (WVU Department of Mathematics).

Lastly, I dedicate this work to my lovely wife Omotade Ademoye, my adorable son, Foluso Ademoye, and my siblings. I appreciate their love, supports and prayers. 


\section{Contents}

Acknowledgements $\quad$ iii

List of Figures $\quad$ vi

List of Tables $\quad$ ix

\section{Contents}

Chapter 1

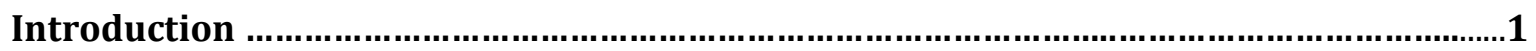

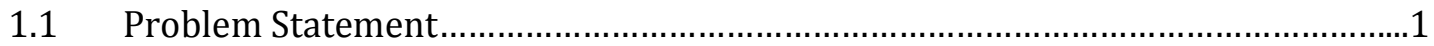

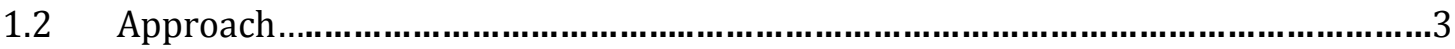

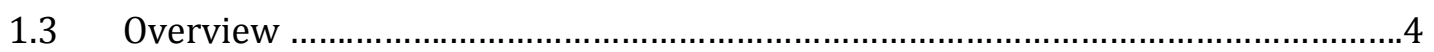

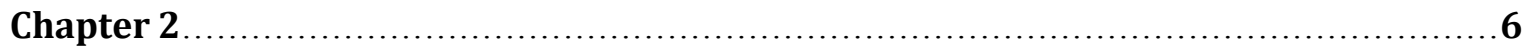

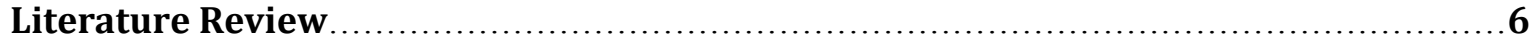

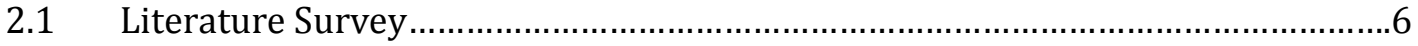

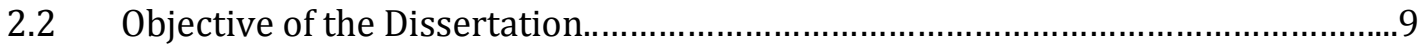

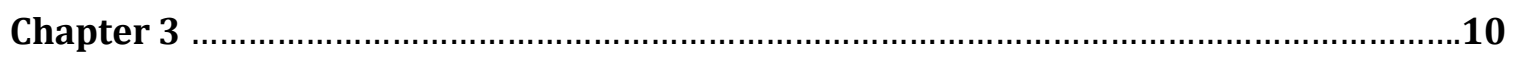

Synergetic Control Design Approach and Application to Power Systems......................10

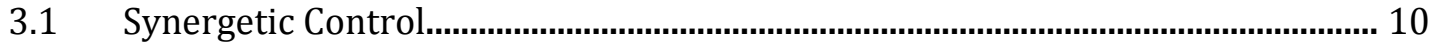

3.2 Procedure of Synergetic Control ................................................................................. 11

3.3 Power System Problem Formulation..............................................................24

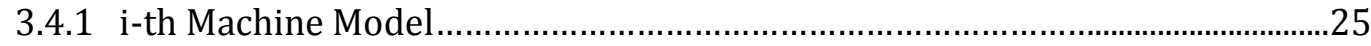

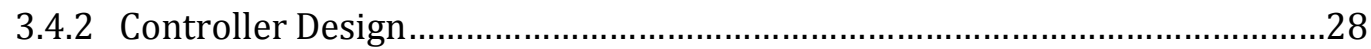

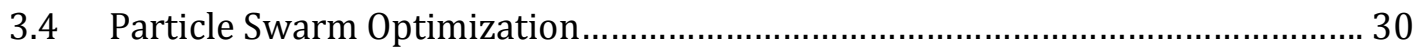

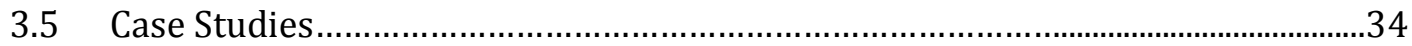

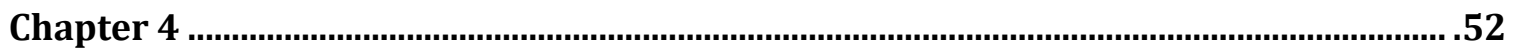

Coordination of Synergetic Excitation Controller SVC Damping Controller ..................52

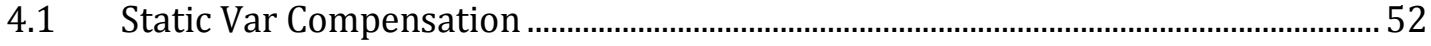

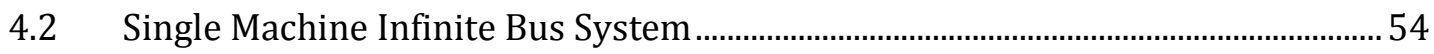

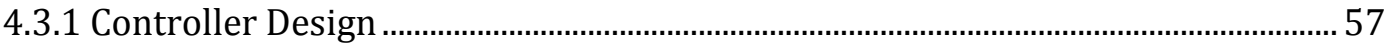

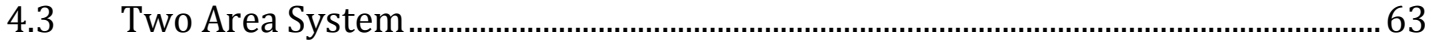

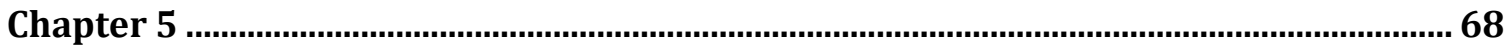


Decentralized Synergetic Control With Variable Parameters .....................................68

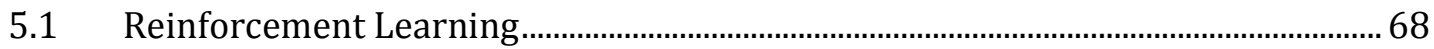

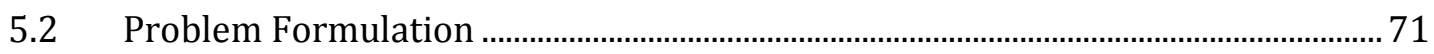

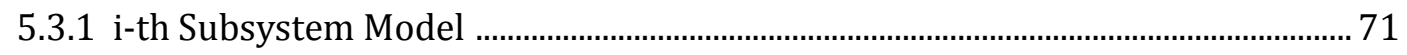

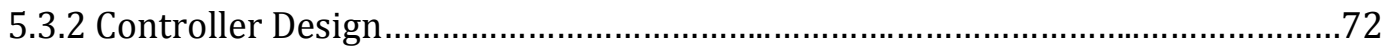

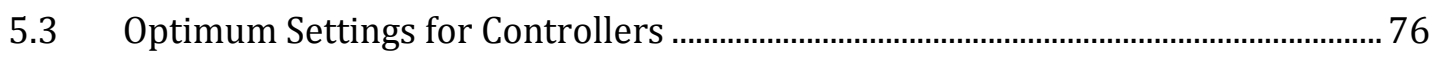

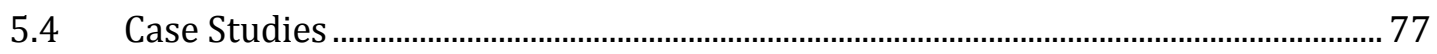

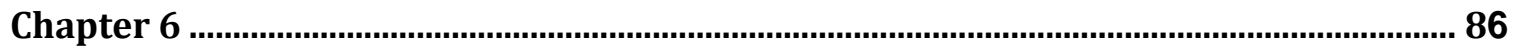

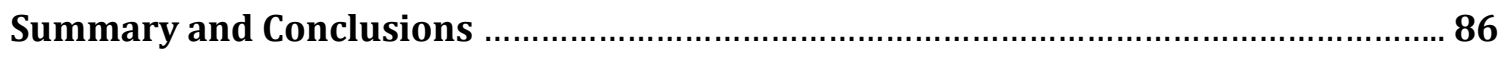

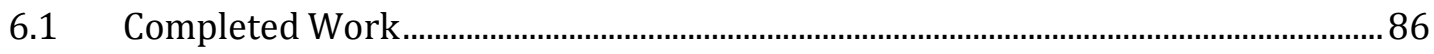

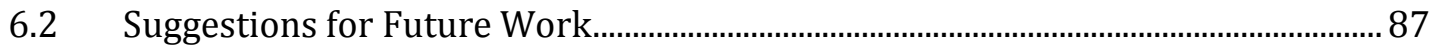

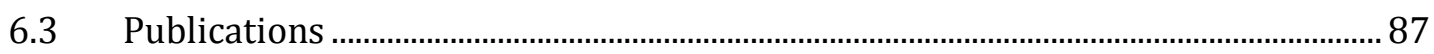

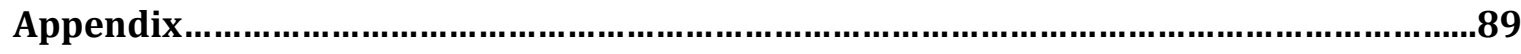

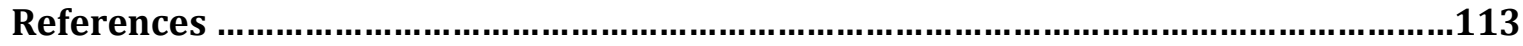




\section{List of Figures}

Figure 3.1: Trajectory of variable $x_{2}$ for different values of $K$

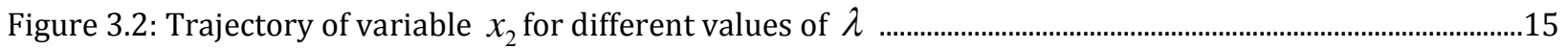

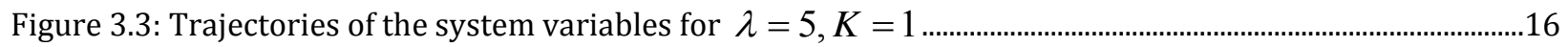

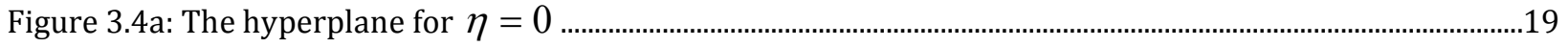

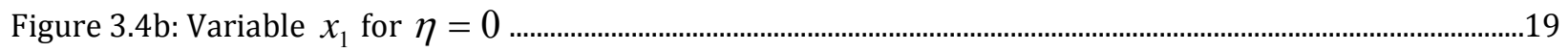

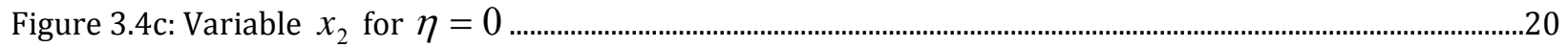

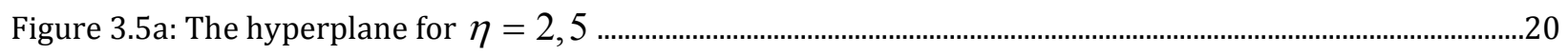

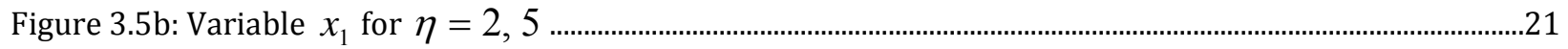

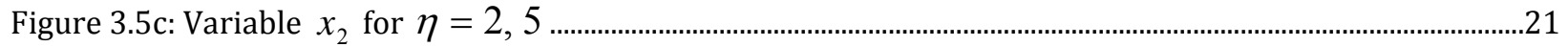

Figure 3.6: System response when the control law synthesized by using synergetic control approach is

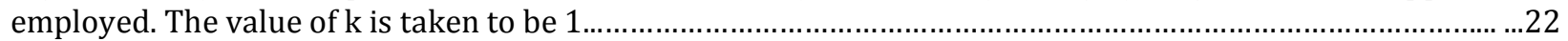

Figure 3.7: System response when the control law synthesized by using synergetic control approach is

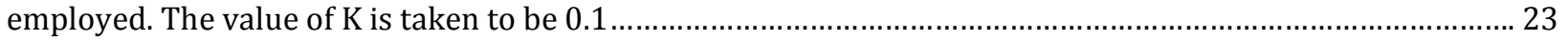

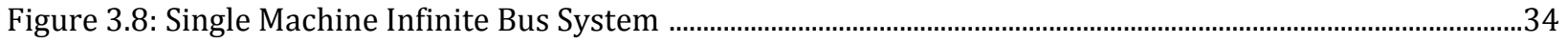

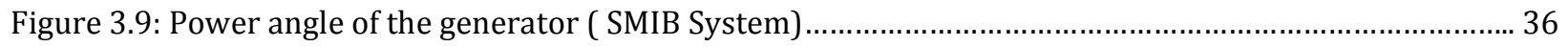

Figure 3.10: Angular speed of the generator ( SMIB System) …...............................................................................36

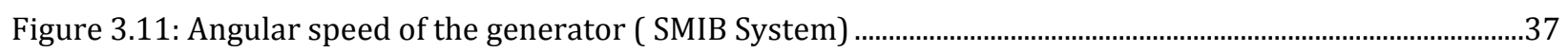

Figure 3.12: Three Machine Nine Bus Power System ................................................................................................

Figure 3.13: Relative rotor angle (degree) 2-1 (3-Machine Nine Bus System) ........................................................39

Figure 3.14: Relative rotor angle (degree) 3-1 (3-Machine Nine Bus System) ........................................................39

Figure 3.15: Speed of generator 1 (3-Machine Nine Bus System) .............................................................................

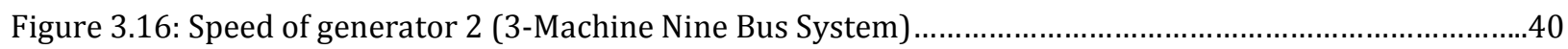

Figure 3.17: Speed of generator 3 (3-Machine Nine Bus System) ..............................................................................41

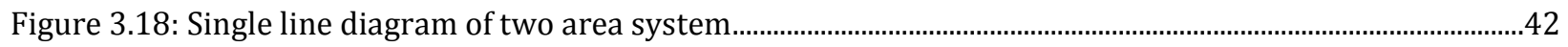

Figure 3.19: Relative rotor angle (degree) 3 - 1for case I (TAS) .................................................................................43

Figure 3.20: Relative rotor angle (degree) 4 - 1for case I(TAS) …................................................................................

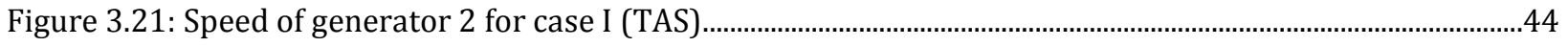

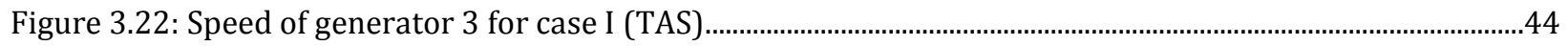

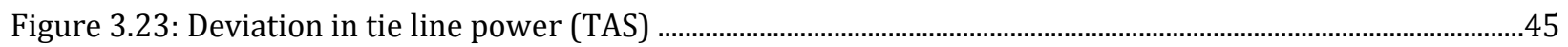

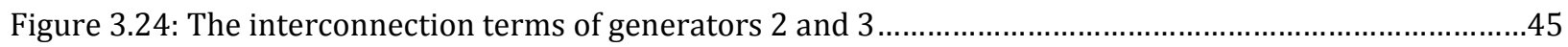

Figure 3.25: Relative rotor angle (degree) 3 - 1 for case II (TAS) ............................................................................46 


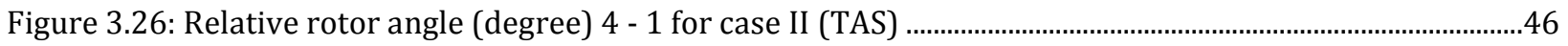

Figure 3.27: Synergetic excitation control signals for Generators 2 and 3 ..................................................................47

Figure 3.28: Fifty Machine System Single Line Diagram..............................................................................................

Figure 3.29: Relative rotor angles (degree) 6 - 1, 2 - 6 (Fifty Machine System)..........................................................49

Figure 3.30: Relative rotor angles (degree) 2 - 1 (Fifty Machine System) ……………………………………..........49

Figure 3.31: Excitation control signals for Generators2 and 6 (Fifty Machine System)..........................................50

Figure 3.32: Terminal voltages of Generators 2 and 6 (Fifty Machine System) .....................................................50

Figure 4.1: SVC Equivalent Circuit Model..............................................................................................................................

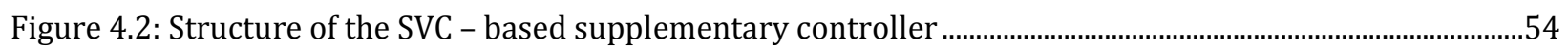

Figure 4.3: Single Machine Infinite Bus System with SVC …………………………....................................................5

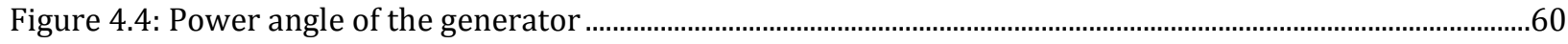

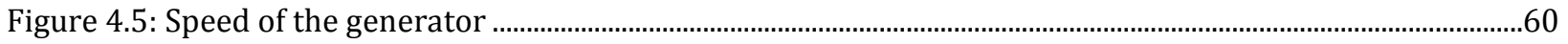

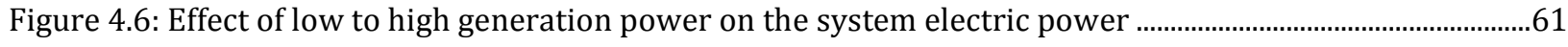

Figure 4.7: Effect of low to high generation power on speed of the generator ...........................................................61

Figure 4.8: Effect of low to high generation power on terminal voltage of the generator ........................................62

Figure 4.9: Two Area System with SVC device ................................................................................................................62

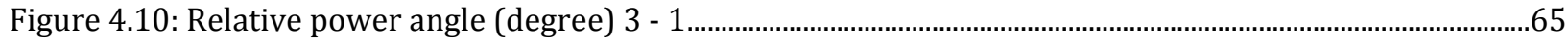

Figure 4.11: Relative power angle (degree) 4 - 1......................................................................................................65

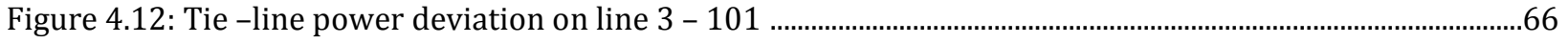

Figure 4.13: Angular speed of generator 3 ………….................................................................................................66

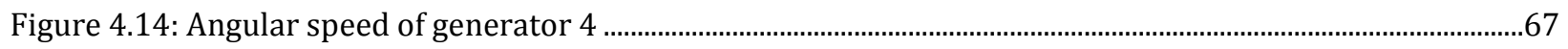

Figure 5.1: Two area system ..........................................................................................................................................

Figure 5.2: Relative angle $\delta_{31}$ under Operating Condition I ……………...................................................................79

Figure 5.3: Relative angle $\delta_{41}$ under Operating Condition I .....................................................................................79

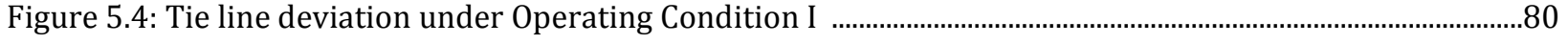

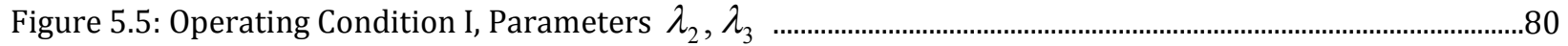

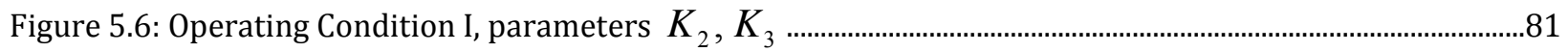

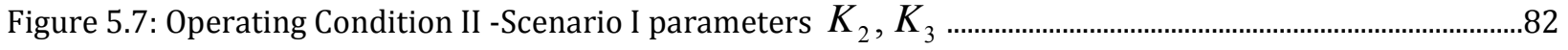

Figure 5.8: Relative angle $\delta_{31}$ under Operating Condition II Scenario I ...................................................................82

Figure 5.9: Relative angle $\delta_{41}$ under Operating Condition II Scenario I.......................................................................83

Figure 5.10: Relative angle $\delta_{31}$ under Operating Condition II Scenario II ....................................................................83

Figure 5.11: Relative angle $\delta_{41}$ under Operating Condition II Scenario I ………………………............................84

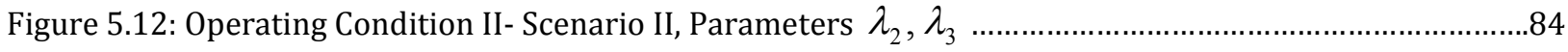




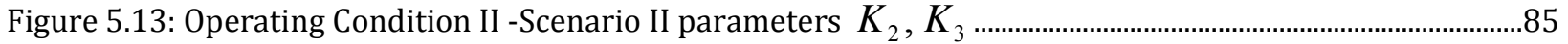




\section{List of tables}

3.1: Synchronous parameter and transmission line variables ...............................................................26

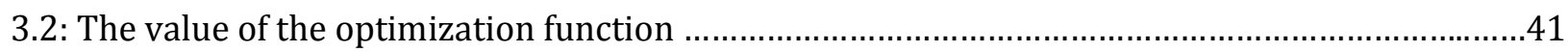

3.3: Parameters for synergetic Controllers ……................................................................................... 48

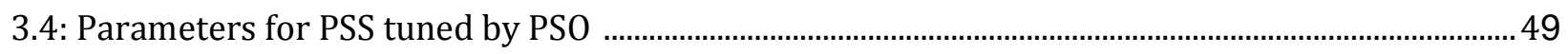

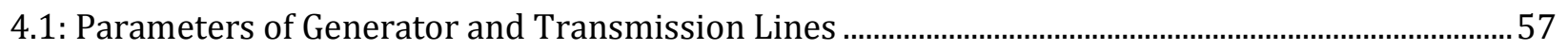

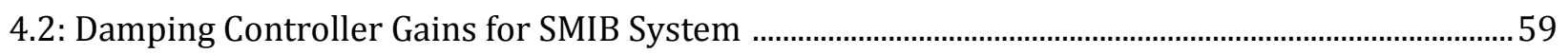

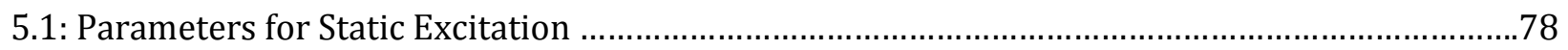

5.2: Optimum Parameters of the Controllers …….................................................................................... 78 


\section{Chapter 1}

\section{Introduction}

\subsection{Problem Statement}

The electric power system is known to be one of the most intrinsic complex interconnected systems that constantly changes in structure as a result of disturbances, load changes, faults and addition of new devices or equipment. The quality of life of the citizens of any nation has often been associated with the amount of electric power consumption of that nation and electricity is known to play a major role in the growth and sustenance of its development and because of this significant importance, it is expedient to guarantee the reliability and stability of the system. Due to more stringent regulation reforms and more demand for electricity, the complexity of electric power system has increased stupendously and many of its components are forced to operate at points close to their stability limits. Also, due to increase in complex nature, a power system is considered to be a large-scale multiple-input multiple output system, which comprises of subsystems that are distributed over a large geographical area. This makes the objective of stabilizing the entire system a difficult task [1]. To maintain a stable and reliable service, the electric grid must remain rigid, unperturbed and capable of withstanding a variety of disturbances. This has been a crucial concern when considering or evaluating the behavior of a power system.

According to IEEE / CIGRE joint task on Stability Terms and Definitions [2], power system stability is defined as the ability of an electric power system, for a given operating condition, to recover or regain a state of operating equilibrium after subjection to a physical disturbance, with most system variables bounded so that the entire system remain intact. These disturbances might be due to load changes, loss of long transmission line or trip of large generator(s) from the electric grid.

The ability of the power system to maintain synchronism when exposed to a severe transitory disturbance is known as transient stability. Stability depends on both the initial operating state of the system and the severity of the disturbance. Transient stability analysis examines the dynamic 
behavior of power system electrical networks, electrical loads, and electro-mechanical equations of motion of the interconnected generators for as much as several seconds following a disturbance. This analysis is done using many levels of modeling details. Mathematical formulation is governed by differential algebraic equations (DAE) that represent the dynamics of the generators in connection with the grid. These generators are electromechanical systems that are required to run in synchronism. Under normal operating conditions, an electrical power system is near equilibrium, with only minor deviations from true-steady state conditions caused by small variations in the loads.

Due to large disturbances on the power system, such as a short circuit, there are significant, almost instantaneous, variations in the rotor speeds of some generators in the system. Consequently, lightly damped electro-mechanical modes of oscillation may be exacerbated. During the oscillations activated by these modes, kinetic energy of rotating masses is exchanged between the generators while electric power flows through the network [3]. The power swings further produce oscillations in voltages and currents which can lead to damage of equipment or disruption in monitoring devices if the oscillation is sufficiently large. Oscillations in voltages can also lead to voltage limit violations causing protective devices to trip and forcing equipment outages. Two types of oscillations are of more concern [3]:

- Local modes or machine-system modes are associated with the swinging of the units at a generating station with respect to the rest of the power system. The term local is used because the oscillations are localized at one station or a small part of the power system, typically with a frequency of $1-2 \mathrm{~Hz}$.

- Interarea modes are associated with the swinging of many machines in one part of the system against machines in the other parts. They are caused by two or more groups of closely coupled machines being interconnected by week ties. Typical frequency range of such oscillations is $0.1-1 \mathrm{~Hz}$.

Control is an important element to maintain the stability of an interconnected power system. Power system controls greatly affect the damping of electric power angle oscillation. Because of the necessity of guaranteeing power system stability and the effort of the control elements in achieving that, numerous form of control structures or/and techniques are being explored and employed. Control elements can be installed on generators, transmission lines, and distribution side. In this dissertation, elements (controllers) are installed on generators and transmission lines to ensure the stability of the entire grid network. 
As the electric power system is growing rapidly, the dynamic performance of the controls, and the method of analyzing the stability of the system is also evolving. The design and implementation of control techniques to improve the stability of large scale power systems is becoming more intriguing and challenging.

One way of improving system stability is via excitation system [4]. The excitation system can regulate the terminal voltage of the generator when it is used with an automatic voltage regulator (AVR). With supplementary control, excitation system can also render oscillation damping to power systems. Another way of enhancing system stability is via the use of Flexible Alternating Current Transmission (FACTS) devices [5]. They are based on high-voltage and high-speed power electronics devices. They increase the controllability of power flows and voltages improving the utilization and stability of existing systems. The range of FACTS devices includes thyristor based applications, e.g. Static Var Compensator (SVC) and Thyristor-Controlled Series Capacitor (TCSC) and the conventional High-Voltage Direct Current (HVDC) transmission systems, and Gate Turn-off (GTO) based applications, e.g., Static Synchronous Compensator (STATCOM). The FACTS device utilized in this work is SVC.

Most control techniques available for designing excitation's supplementary controller of power systems are based on linearization of the system's model around an operating region. This may not provide a pragmatic solution to the stability problem. A significant large disturbance may poise danger to the system stability because the nonlinearities in the system cannot be adequately compensated for. Hence, the need for nonlinear control designs tools. Complete centralized control scheme is also infeasible due to difficulties in transfer of information among various components of the system because of the physical distance, which may lead to unnecessary increase in the cost of transmission of electricity [24]. Hence, the needs for a decentralized control scheme. Design of controller using nonlinear control techniques is becoming popular as a result of its ability to improve control performance beyond what can be accomplished with linear control techniques.

\subsection{Approach}

The control methodology adopted in this dissertation is as follow. On the excitation control, we designed supplementary controller using synergetic control technique to enhance the transient 
response of the power system. The method has been previously applied to a SMIB system [33], and it was shown through simulation that the controller gives a better performance than the conventional power system stabilizer. Also, the method has good robust characteristic like that of the popular Sliding Mode Control (SMC) technique. But the latter technique introduces steady state chattering effect which can cause wear and tear in actuating system. This gives the proposed technique a major advantage over the SMC. In this dissertation, the technique is adopted to develop decentralized controllers for multi machine power systems. Firstly, each generator in the system is first considered as a subsystem within the entire electric power system. Mathematical model of each subsystem is achieved by a set of algebraic differential equations (ADE), which also include coupling among subsystems. The coupling term between each subsystem and the rest of the system is assumed to be a polynomial function of the active power generated by that subsystem. Synergetic control technique is then employed to each subsystem. The parameters of this controller are properly tuned with the aid of the Particle Swarm Optimization (PSO) technique.

To further enhance the performance of the system by widening its range of operation, Reinforcement Learning (RL) technique is used to vary the gains of the decentralized synergetic supplementary controller in real time. Instead of having pre-selected constant parameters for the controller, the optimum values of the parameters are obtained at first using PSO, and then some of these parameters are re-adjusted through RL. The approach is illustrated with several case studies including a SMIB system with or without a Static Var Compensator (SVC), a Two Area System (TAS) with or without an SVC, a three -machines-nine-bus system and a fifty machine system. Results show that the proposed control technique provides better damping than the conventional power system stabilizers

\subsection{Overview}

This dissertation consists of the following chapters:

- Chapter 1: Introduction

- Chapter 2: Literature Review

- Chapter 3: Synergetic Control Approach and Application to Power Systems

The chapter presents an overview of synergetic control technique. Comparison is made between this technique and sliding mode technique. The technique is employed to design decentralized excitation supplementary controller to enhance the transient stability of 
power systems and the designed controller was implemented on a Single Machine Infinite Bus (SMIB), three machine nine bus, two area and fifty machine systems.

- Chapter 4: Coordination of Synergetic Excitation and SVC Damping Controllers This chapter presents a way of improving the performance of the designed synergetic controller by the addition of Static Var Compensator (SVC) device to a node in the system, designing a supplementary controller for this device and simultaneously tuning the gains of the two controllers using PSO technique. The technique is employed successfully on two area, and SMIB systems.

- Chapter 5: Decentralized Synergetic Control with Variable Structure

Reinforcement learning scheme is presented and employed to vary some of the gains of the synergetic controller. The results show improvement in the performance of the controller for generator exciter in a multi machine system.

- Chapter 6: Summary and Conclusion

This chapter summarizes the merit of the developed nonlinear control and the improvements achieved by designing nonlinear decentralized damping controls using the proposed technique. Suggestion for future work is also provided.

- Appendix: A-D include system benchmark data, and E includes MATLAB computer codes.

All case studies presented in the transient stability enhancement part are performed with the help of the Power Analysis Toolbox (PAT), a simulation package developed by the Advanced Power and Electricity Research Center (APERC) at West Virginia University [46]. 


\section{Chapter 2}

\section{Literature Review}

In this chapter, a literature survey of previous studies relating to damping of oscillation in electric power systems is presented. The survey is organized as follow. In section 2.1 work related to transient stability enhancement is presented. In section 2.2 the objective of this dissertation is detailed.

\subsection{Literature Survey}

The prevention of electric power systems from loss of synchronism after the occurrence of a large disturbance is of great interest. Stability study of power system oscillations depicts that power systems comprise of many modes of oscillation as a result of interactions among various components of the system. Many of these oscillations are due to swinging of the rotor of some of the synchronous generators in the system. If these oscillations are not quickly damped, the stability of the system can be loss. One pragmatic way of enhancing the stability of synchronous generator is via excitation system equipped with supplementary controller [3]. Excitation control provides efficient voltage control and ensures system's stability when subjected to large disturbance.

Supplementary control is often added to actuators in power system to improve the dynamic performance of the system. It gives additional input signal to actuator (exciter or FACTS devices) to dampen oscillations in power systems. The initial notions of supplementary control were established in [10]. The most popular or proven one is the Power System Stabilizer (PSS). It consists of wash-out and lead-lag stages. The wash-out stages, comprising of a stabilizer gain and a time constant (also known as wash-out time constant), acts to bring the steady state of the input signal of the PSS to zero. The lead-lag stage (comprising of two first order phase compensation) on the other hand provides component of electric torque in phase with speed deviation. The most 
common input to this controller are shaft speed deviation, integral of electric power and the terminal frequency [3]. These inputs are local measurements of each generator and thus the controller can operate without the need for remote signals from other generators.

The performance of a PSS depends on the values of its parameters. Many researchers have been focusing on ways of tuning these parameters to aid the effectiveness of the controller. Most of the tuning approaches are off-line, many of which are based on eigen value analysis where linearized state space model of the system are first obtained, and then computation is done based on the model [11]. In [12], frequency domain robust control is employed to design new PSS structure. The approach is based on unstructured uncertainty model which make use of optimization over a class of stabilizing controllers to force a closed loop transfer function to be as close as possible to the desired one [13]. Linear adaptive control has been implemented in [14] for retuning the PSS parameters. And in recent years, more sophisticated off-line tuning techniques based on evolutionary algorithms have been developed for tuning controller parameters. The advantages have the merit of simultaneously tuning the parameters through the framework of multi-objective optimization problem. Genetic Algorithms (GA) have been employed in [15, 16], Tabu Search (TS) optimization technique in [17], and Particle Swarm Optimization (PSO) in [18]. Also in recent years, Reinforcement Learning (RL) technique is utilized for on-line tuning of gains of conventional PSS $[19,20]$.

The control schemes discussed above only deal with small disturbances about an operating point. For large disturbances the pre-fault, fault on, and post fault operating points may differ and in spite of the success achieved through the course of the researches listed above, PSS may not perform properly during certain contingencies. Also, because of the system to be controlled (power system) is highly nonlinear in nature; the need for developing nonlinear control algorithms is growing immensely.

Many nonlinear control schemes have been applied previously with various degrees of accomplishment. Some of them are the direct feedback linearization techniques [21,22] in which the nonlinearity terms is canceled using some form of transformation. To perform this, a large control effort is usually required. Application of direct feedback linearization technique is also extended to multi machine power system [23]. To obtain decentralized controller, the interconnection bounds between generator and the rest of the system is considered to be a polynomial function of absolute rotor angle and speed deviation. Simple linear relationship between excitation field voltage and internal voltage of the generator is assumed in order to obtain 
bound on the coupling term. The proposed technique is implemented on two machine infinite bus system with objective of maintaining stability of the system under occurrence of large fault.

Backstepping method has also been employed with great success [24, 25]. In [24], backstepping with Particle Swarm Optimization tuning technique is presented. Backstepping controller with variable gains is developed and tested on a two area system in [25]. Although a decentralized backstepping controller is designed for each generator, the gains were varied using a centralized reinforcement learning algorithm which makes the entire control structure not to be completely decentralized.

A methodology based on the sliding mode technique is proposed in [26]. The basic notion of the technique was established in [27]. The technique involves defining or finding a stable and invariant sliding surface (or hyperplane) and synthesizing a controller to steer the motion of the system onto the surface. The dynamical behavior of the system when confined to the surface is known as ideal sliding surface .The major advantages of this technique are twofold: reduction in order of the system, and the insensitivity of the motion of the system, after being confined to the surface, to parameter variations implicit in the input channel [28]. Despite good robust characteristic, this control usually introduces steady state chattering effect that can cause wear and tear in actuating system.

Another concept similar to the sliding mode technique is the novel synergetic control technique. The techniques are similar in the steps taken to synthesize the control signal: constructing a stable surface, and designing a controller to force system trajectories to this surface. But they differ in the manner the trajectories are being forced to reach the surface. In the case of sliding mode, the trajectories are forced to reach the surface within a finite period of time. This introduces discontinuity into the control function. But in the case of synergetic control, the transition is done exponentially (in a smoothen version). This eliminates the problem of chattering that is a major setback for sliding mode technique. However, it may take a bit longer period of time for a system with synergetic controller to stabilize.

Some major works that have been previously done with this technique are: the analysis of synergetic control approach is detailed in [29] and the advantage of the method over sliding mode approach is given in [30], the synergetic control strategy is developed for nonlinear speed control of a Permanent Magnet (PM) synchronous motor for DC-DC boost converters [31, 32], a nonlinear 
power system stabilizer is developed for a single machine infinite bus [33]. None of these papers have addressed the decentralized synergetic control design problem.

Coordination between controllers also plays a crucial role once the controllers are in action at the same time. Following a contingency in the system, lack of coordination of among various controllers acting together may yield unpleasant performance of the entire system. For instance, a system having exciter and FACTS device like SVC may behave poorly or destabilize if the two supplementary controllers (for the exciter and the SVC) are not properly coordinated. Some works on coordination of SVC and exciters are presented in [34,35]. The approaches employed in this work are based on a linearized model of the system which may not adequately capture the complexity in the dynamics of the system.

\subsection{Objective of the Dissertation}

The main concerns addressed in this work are

- Utilize a nonlinear control technique to design excitation's supplementary controllers. The method employed in this work is synergetic control technique.

- Obtain a procedure for optimally tuning the parameters of the designed controller

- Implement the proposed controller on a Single Machine Infinite Bus system, and multi machine systems

- Coordinate the supplementary controllers to the excitation and SVC systems by simultaneously tuning their parameters.

- Enhance the stability of the system under different operating conditions. This is achieved by varying some of the parameters of the synergetic controller.

- Compare the proposed control performance with the existing power system stabilizer (PSS) 


\section{Chapter 3}

\section{Synergetic Control Design Approach and Application to Power Systems}

In this chapter, the technique of synergetic control design is introduced in 3.1. The procedure of obtaining the control law is given in 3.3. Three examples are given to show the effectiveness of the technique. The application of the technique to power systems is illustrated from 3.2 to 3.5. Problem formulation for generator is given in 3.3. Particle Swarm Optimization, PSO, technique for function optimization is discussed in detail in 3.4. The purpose of this technique is to obtain optimum set of gains for synergetic controller. Single machine infinite bus, three machine nine bus test system, two area system and fifty machine system are presented in section 3.5 for implementation of the proposed techniques.

\subsection{Synergetic Control}

Synergetic control was developed by Kolesnikov and his coworkers [36]. It is a progressive approach in the area of control systems. The approach is often referred to as the stabilization and control via system restriction and manifold invariance [29]. The major idea of the concept is to confine the motion or trajectories of the system to a manifold (or hyperplane). This includes forcing the system onto the manifold in case it is not on the manifold already. The control problem addressed in this work is basically stability (i.e. damping any oscillation that may arise due to disturbance in the system) and since the system is forced by the synergetic controller to take the characteristics of the manifold, the manifold has to be constructed in such a way to ensure that the closed loop system behavior is stable. The design approach can thus be said to comprise of two major steps: the construction of a stable manifold (or hyperplane); and the synthesis or design of a controller so that the trajectories of the system are forced onto, and subsequently remain on, the hyperplane. The objective of the controller can be seen as the one that causes the manifold to be invariant and attractive. 
The technique of synergetic control is similar to that of Sliding Mode Control (SMC) in the way the manifold is being constructed. However, it differs from SMC technique in the manner the system is forced to reach the manifold. In the case of SMC, the system is forced to reach the manifold in a finite period of time which introduces some form of discontinuities in the control action and thus creating chattering on the manifold. This chattering can cause wears and tears in the actuating components of the system. But in the case of synergetic control, the system is caused to reach the manifold in an exponential manner and thus removing chattering effect.

\subsection{Procedure of Synergetic Control}

As mentioned in the previous section, the synergetic control design procedure follows the Analytical Design of Aggregated Regulators (ADAR) method. The steps involved in the control design are summarized as follow [30]

Assume the system to be controlled is delineated by a set of nonlinear equation of the form:

$\dot{x}=f(x, u)$

where $x$ and $u$ are the system state variable vector and the input vector respectively. The procedure is started by defining a macro-variable as a function of the state variables:

$\varphi=\varphi(x)$

The characteristic of the macro-variable can be selected in accordance to the control specifications. For example, to regulate or stabilize the output of the system. The manner the macro-variable is defined or formulated determines how the system behaves once it reaches the manifold. The process can be repeated to formulate as many macro-variables as the number of control channels

(for instance, two macro-variables are required for a system with two input channel). It has been shown in [29], that (3.3) is a solution of the optimum problem whose objective function is given in (3.4)

$K \dot{\varphi}+\varphi=0$ 


$$
J=\int_{t_{0}}^{t_{f}} L(t, \varphi, \dot{\varphi})
$$

where $\left\{t_{0}, t_{f}\right\}$ are the initial and final time, and $L$ is defined as:

$$
L(t, \varphi, \dot{\varphi})=\dot{\varphi} K^{T} K \dot{\varphi}+\varphi^{T} \varphi
$$

and $K$ is a design parameter that specifies the time it takes the macro variable to converge to zero or evolve into the manifold, which can also be viewed as the speed at which the system variable reaches the manifold. By substituting (3.1) and (3.2) into (3.3) we can obtain

$K \frac{\partial \varphi}{\partial x} \dot{x}+\varphi=K \frac{\partial \varphi}{\partial x} f(x, u)+\varphi=0$

By defining an appropriate macro-variable and choosing the parameter $K$, the control output can be derived from (3.6).

There is no unique way of constructing the manifold. The manner the manifold is constructed depends on the type of problem (regulation or tracking) and determines the quality of the performance of the controller. The method adopted in this work, the same as the one adopted in Slotine [37], is illustrated with the help of the examples given below.

Example 1: Consider a linear time invariant system whose dynamics is defined by the state space given below:

$$
\begin{aligned}
& {\left[\begin{array}{l}
\dot{x}_{1} \\
\dot{x}_{2}
\end{array}\right]=\left[\begin{array}{ll}
a_{11} & a_{12} \\
a_{21} & a_{22}
\end{array}\right]\left[\begin{array}{l}
x_{1} \\
x_{2}
\end{array}\right]+\left[\begin{array}{l}
b_{1} \\
b_{2}
\end{array}\right] u} \\
& y=x_{1}
\end{aligned}
$$

Assuming we want to move the state of the system from any initial point $\left(x_{10}, x_{20}\right)$ to a location of particular interest $\left(x_{1 f}, x_{2 f}\right)$. This is known to be a regulatory problem. New variables known as error variables, are defined as: $\bar{x}_{1}=x_{1}-x_{1 f}$, and $\bar{x}_{2}=x_{2}-x_{2 f}$. And (3.7) can be re-written as: 
$\left[\begin{array}{l}\dot{\bar{X}}_{1} \\ \dot{\bar{X}}_{2}\end{array}\right]=\left[\begin{array}{ll}a_{11} & a_{12} \\ a_{21} & a_{22}\end{array}\right]\left[\begin{array}{l}\bar{x}_{1} \\ \bar{x}_{2}\end{array}\right]+\left[\begin{array}{l}b_{1} \\ b_{2}\end{array}\right] u+\left[\begin{array}{l}f_{1} \\ f_{2}\end{array}\right]$

where

$$
\begin{aligned}
& f_{1}=-\left(a_{11} x_{1 f}+a_{12} x_{2 f}\right) \\
& f_{2}=-\left(a_{21} x_{1 f}+a_{22} x_{2 f}\right)
\end{aligned}
$$

The macro variable is usually defined as a polynomial differential function of the error variable. The degree of the polynomial is $n-1$ with $n$ being the order of the system i.e. in general case; the macro variable is defined as:

$$
\varphi=\left(\frac{d}{d t}+\lambda\right)^{n-1} \bar{x}_{1}
$$

In the case of the system defined by the equation in (3.7) or (3.8), the micro variable is defined as:

$$
\varphi=\dot{\bar{x}}_{1}+\lambda \bar{x}_{1}
$$

The solution to (3.6) is given as:

$$
\varphi=\varphi(0) e^{-\frac{1}{K} t}
$$

where $\varphi(0)$ is the initial value of the macro variable. We can also see that as $t \rightarrow \infty, \varphi=0$, which when substituted into (3.12) will produce a manifold given by:

$$
\varphi=\dot{\bar{X}}_{1}+\lambda \bar{x}_{1}=0
$$

For the manifold defined in (3.13) to be stable, $\lambda>0$. The solution of (3.13) will produce, $\bar{x}_{1}=\bar{x}_{2}=0$ and thus $x_{1}=x_{1 f}$ and $x_{2}=x_{2 f}$. Thus, the control input obtained when (3.6) is solved is said to have forced the system to the manifold.

Example 2: Consider a nonlinear system whose dynamics is given by the following differential equation: 
$\dddot{x}+\alpha_{1} \ddot{x}^{2}+\alpha_{2} \dot{x}^{5} \sin 4 x=u$

where $\alpha_{1}$ and $\alpha_{2}$ are known constant parameters. This equation can be written in state space form as:

$\dot{x}_{1}=x_{2}$

$\dot{x}_{2}=x_{3}$

$\dot{x}_{3}=-\alpha_{1} x_{3}^{2}-\alpha_{2} x_{2}^{5} \sin 4 x_{1}+u$

Where $x_{1}=x, x_{2}=\dot{x}$ and $x_{3}=\ddot{x}$ are the state variables. The control design objective for the system (3.15) is to regulate the state to the origin of $\mathbf{R}^{3}$. Using the procedure described in the example above to construct the manifold, we have

$\varphi\left(x_{1}, x_{2}, x_{3}\right)=\left(\frac{d}{d t}+\lambda\right)^{2} x_{1}=\left(\frac{d^{2}}{d t^{2}}+2 \lambda \frac{d}{d t}+\lambda^{2}\right) x_{1}$

The synergetic control law is obtained by solving the first- order differential equation,

$K \dot{\varphi}\left(x_{1}, x_{2}, x_{3}\right)+\varphi\left(x_{1}, x_{2}, x_{3}\right)=0$

Solving this differential equation for $u$, we obtain

$u=\alpha_{1} x_{3}^{2}+\alpha_{2} x_{2}^{5} \sin 4 x_{1}-\left(2 \lambda+\frac{1}{K}\right) x_{3}-\lambda\left(\lambda+\frac{2}{K}\right) x_{2}-\frac{\lambda^{2}}{K} x_{1}$

With $\lambda$ chosen to be 5 , the effect of varying $K$ is showing in Figure 3.1. Figure 3.2 depicts the effect of varying $\lambda$ with $K$ set to 0.1 and Figure 3.3 shows the trajectory of the state variables for $\lambda=5$ and $K=1$. The initial condition of the system is $\{-5,-3,2\}$ and the values of the model parameters $\alpha_{1}$ and $\alpha_{2} x$ are 2.25 and -0.187 respectively. 
We can see from Figure 3.1 that for a constant value of $\lambda$, the settling time increases and the overshoot decreases with increase in $K$, and from Figure 3.2 that for a constant value of $K$, the settling time decreases and the overshoot increases with increase in $\lambda$. Thus, the choice of these two variables has tremendous effect on the performance of the closed loop of the system.

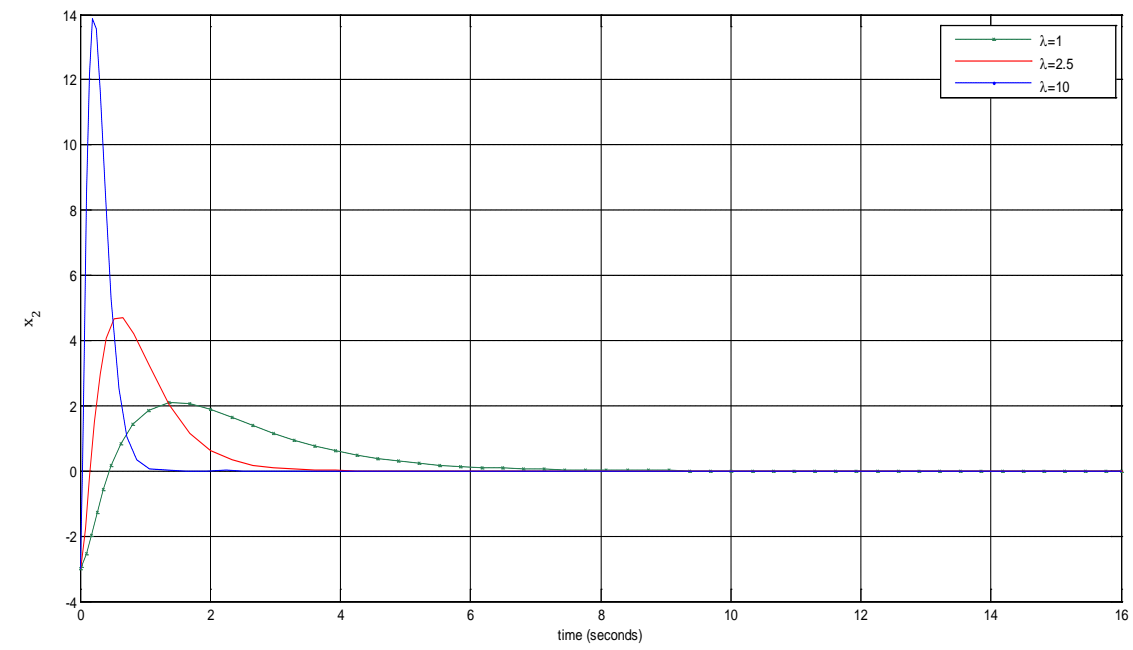

Figure 3.1: Trajectory of variable $x_{2}$ for different values of $\lambda$

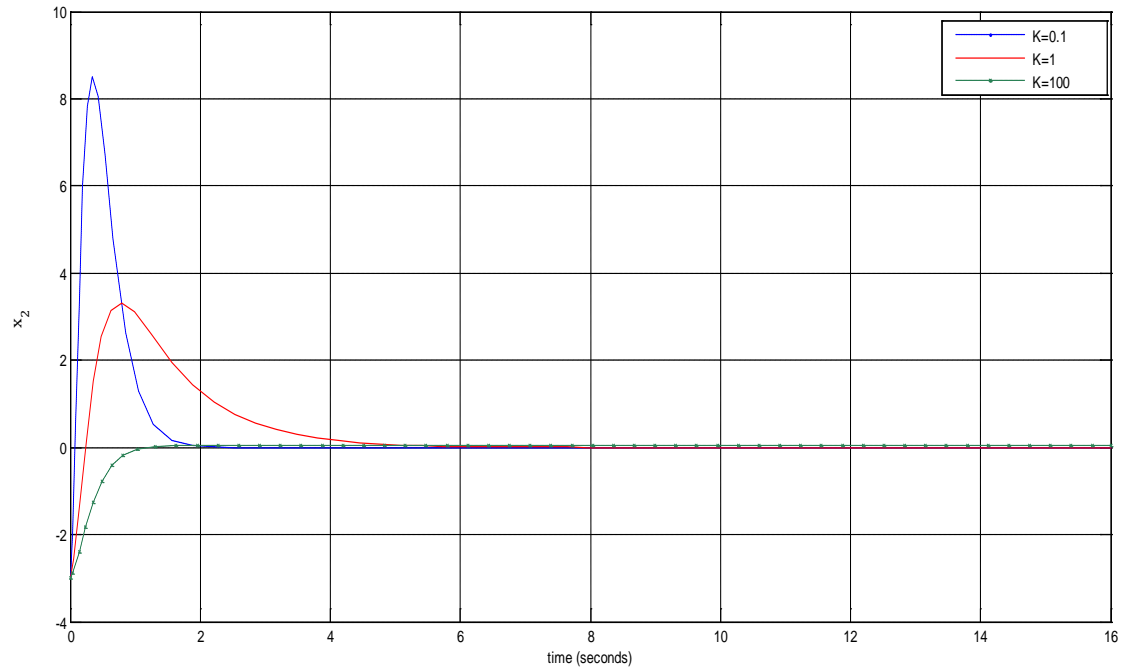

Figure 3.2: Trajectory of variable $x_{2}$ for different values of $K$ 


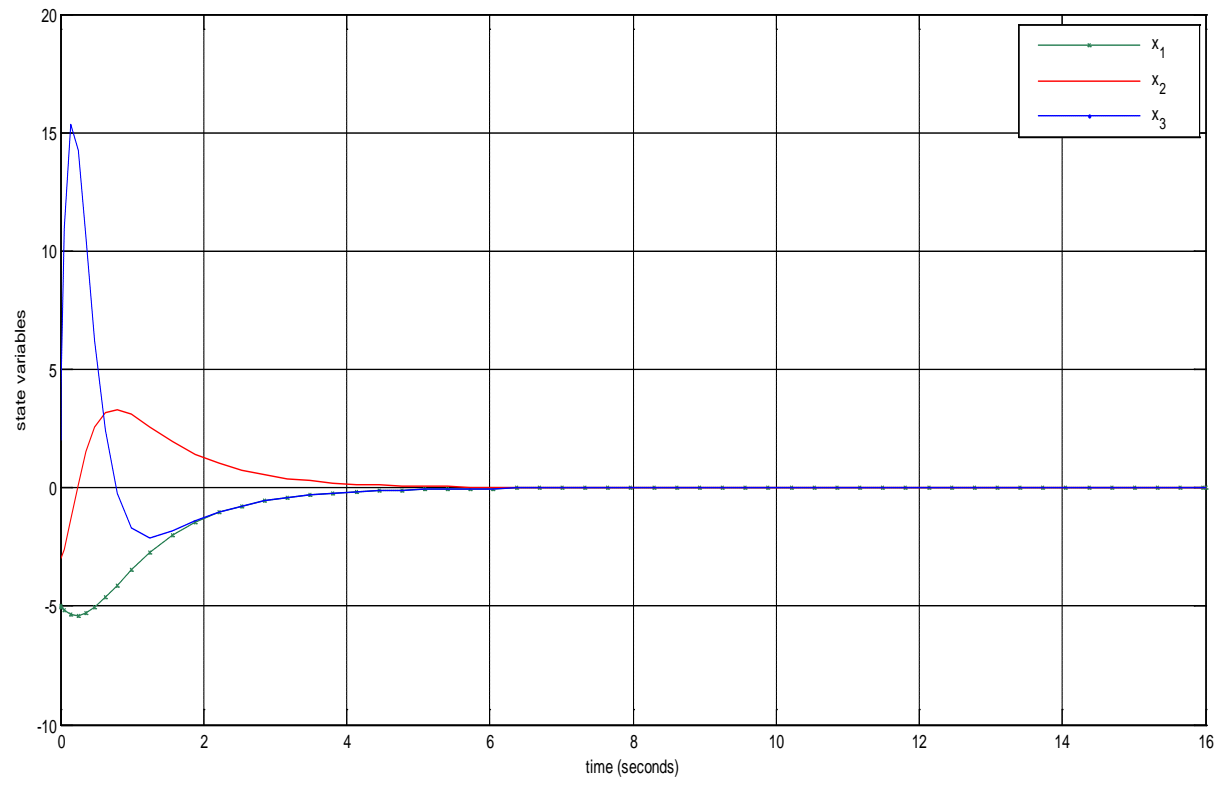

Figure 3.3: Trajectories of the system variables for $\lambda=5, K=1$

As mentioned earlier, the manner in which the macro-variable is formulated depends on the nature of the system we are working with. The next example shows another way of formulating the macro-variable.

Example 3: Consider the nonlinear dynamical system [38]

$\dot{x}_{1}=1+x_{2}+\left(x_{1}-1\right)^{3}, \quad x_{1}(0)=x_{10}$

$\dot{x}_{2}=x_{1}+u, \quad x_{2}(0)=x_{20}$

The objective is to determine a controller that globally stabilizes the system. For this example, both Synergetic Control and Sliding Mode techniques are employed in order to compare the performance of the controllers synthesized by the two approaches.

\section{Sliding Mode Technique}

As it has been stated earlier, this technique involves defining a stable manifold or hyperplane (or a sliding surface), and synthesizing a controller that will force the system onto the manifold. The hyperplane used in this example is defined as: 
$\varphi(x)=\left(\frac{d}{d t}+\lambda\right) x_{1}, \quad \lambda>0$

The controller to be synthesized using this technique consists of two parts: the equivalent and the discontinuous parts i.e. $u(t)=u_{e q}(t)+u_{d}$. Where $u_{e q}$, and $u_{d}$ are the controller's equivalent and discontinuous parts respectively. The equivalent part of the controller is obtained from the solution of (4)

$\dot{\varphi}=0$

Equation (3.23) is known to be the dynamic of the system on the sliding surface. This equation ensures the invariance of the surface i.e. once the trajectories of the system reach the surface; they are bound to remain on it. By solving (3.23) we can obtain $u_{e q}$ to be:

$$
\begin{aligned}
& \dot{\varphi}=\ddot{x}_{1}+\lambda \dot{x}_{1}=\dot{x}_{2}+\left(3\left(x_{1}-1\right)^{2}+\lambda\right) \dot{x}_{1}=0 \\
& \therefore u_{e q}=-x_{1}-\left(3\left(x_{1}-1\right)^{2}+\lambda\right)\left(1+x_{2}+\left(x_{1}-1\right)^{3}\right)
\end{aligned}
$$

The discontinuous or the switching component of the controller is obtained using the $\eta$ reachability condition $[38,2]$. This part of the controller is to guarantee that the trajectories of the system are forced onto the sliding surface. Therefore, the switching part of the controller is given as:

$u_{d}=-\eta \operatorname{sgn}(\varphi)$

$\eta$ is a strictly positive constant. Its value determines the time at which the system's trajectory will reach the surface.

NB: The reachability condition may be written mathematically as:

$\lim _{\varphi \rightarrow 0^{+}} \dot{\varphi}<0 \quad$ and $\quad \lim _{\varphi \rightarrow 0^{-}} \varphi>0$ 
The controller obtained above, i.e. $u=u_{e q}+u_{d}$ will ensure that this condition is satisfied. Define a Lyapunov function for the state $\varphi$ as:

$V(\varphi)=\frac{1}{2} \varphi^{2}$

The derivative of this function will give

$\frac{1}{2} \frac{d}{d t} \varphi^{2} \leq-\eta|\varphi|, \quad$ and integrating from 0 to $t_{f}$, we have

$\left|\varphi\left(t_{f}\right)\right|-|\varphi(0)| \leq-\eta t_{f}$

Therefore, the time it takes the trajectory of the system to reach $\varphi=0$, represented by $t_{f}$, satisfies

$t_{f} \leq \frac{|\varphi(0)|}{\eta}$

Therefore, for this example, the control law that forces the system's trajectories onto the manifold (or sliding surface) and ensures they stay on that surface afterward is given as:

$u=-x_{1}-\left(3\left(x_{1}-1\right)^{2}+\lambda\right)\left(1+x_{2}\left(x_{1}-1\right)^{3}\right)-\eta \operatorname{sgn}(\varphi)$

\section{Synergetic Control Technique}

Using the same macro-variable or hyperplane defined in (3.21), the controller is obtained from the equation of evolution given (3.17). The control law is obtained as:

$u=-\left(1+\frac{\lambda}{K}\right) x_{1}-\left(1+x_{2}+\left(x_{1}-1\right)^{3}\right)\left(3\left(x_{1}-1\right)^{2}+\lambda+\frac{1}{K}\right)$

The simulation of the two control laws, (3.28) and (3.29) are depicted in the Figures 3.4, 3.5, 3.6 and 3.7. The initial states of the system are assumed to be $x_{1}(0)=2, x_{2}(0)=-3$, and the value of $\lambda$ is taken to be equal 5. The control objective is to bring the states of the system to the origin (equilibrium point). To implement the controller expressed by (3.28), two different values of $\eta$ ( $\eta=0,2)$ are used. The first value of $\eta$ is the case when only the equivalent part of the controller is 
used. The simulation result of this case is depicted in Figure 3.4. It can be shown from this Figure that the system's trajectories are parallel to the sliding surface and they will never be able to

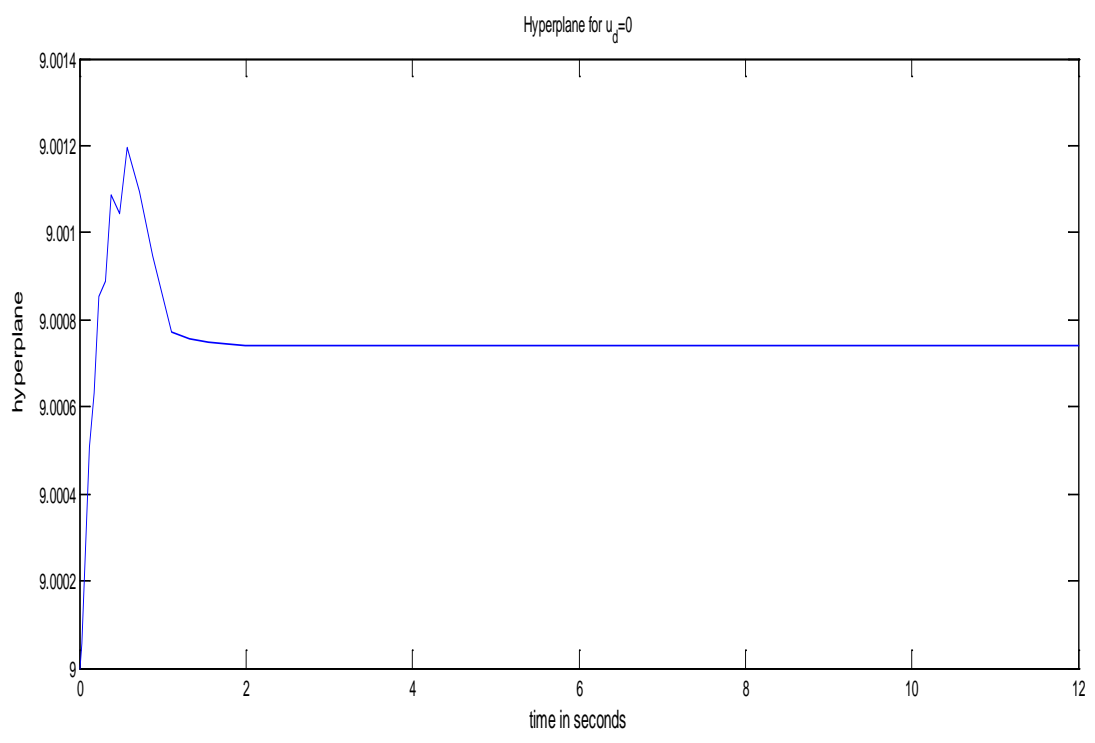

Figure 3.4a: The hyperplane for $u_{d}=0$

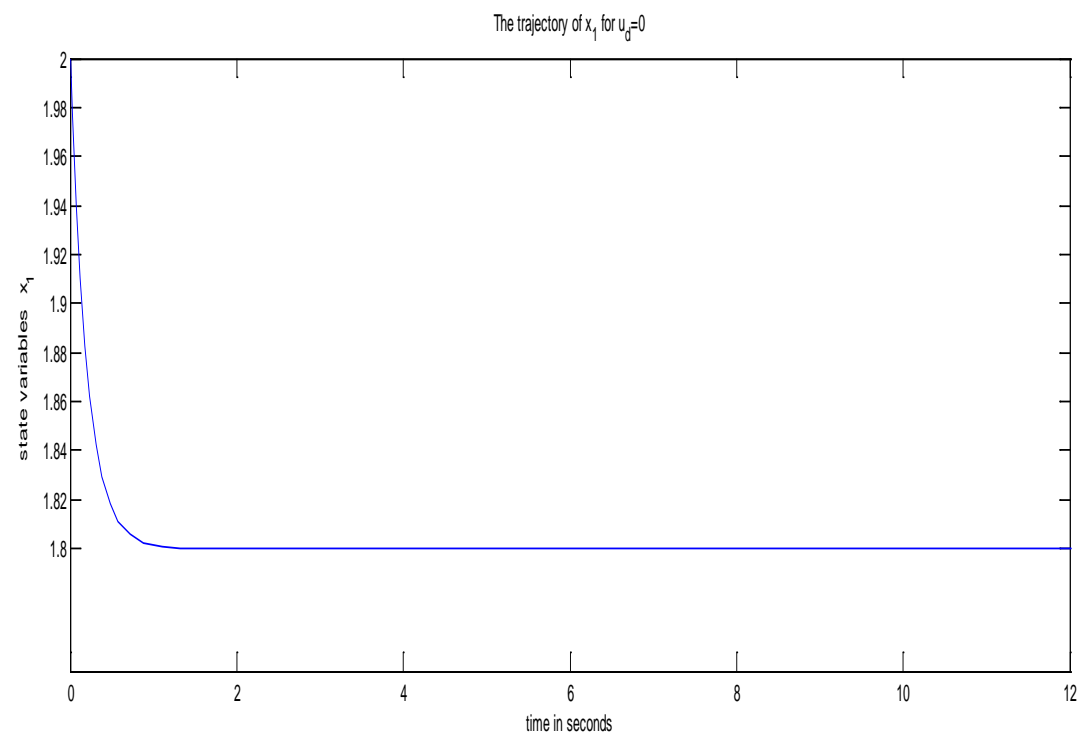

Figure 3.4b: Variable $x_{1}$ for $\eta=0$ 


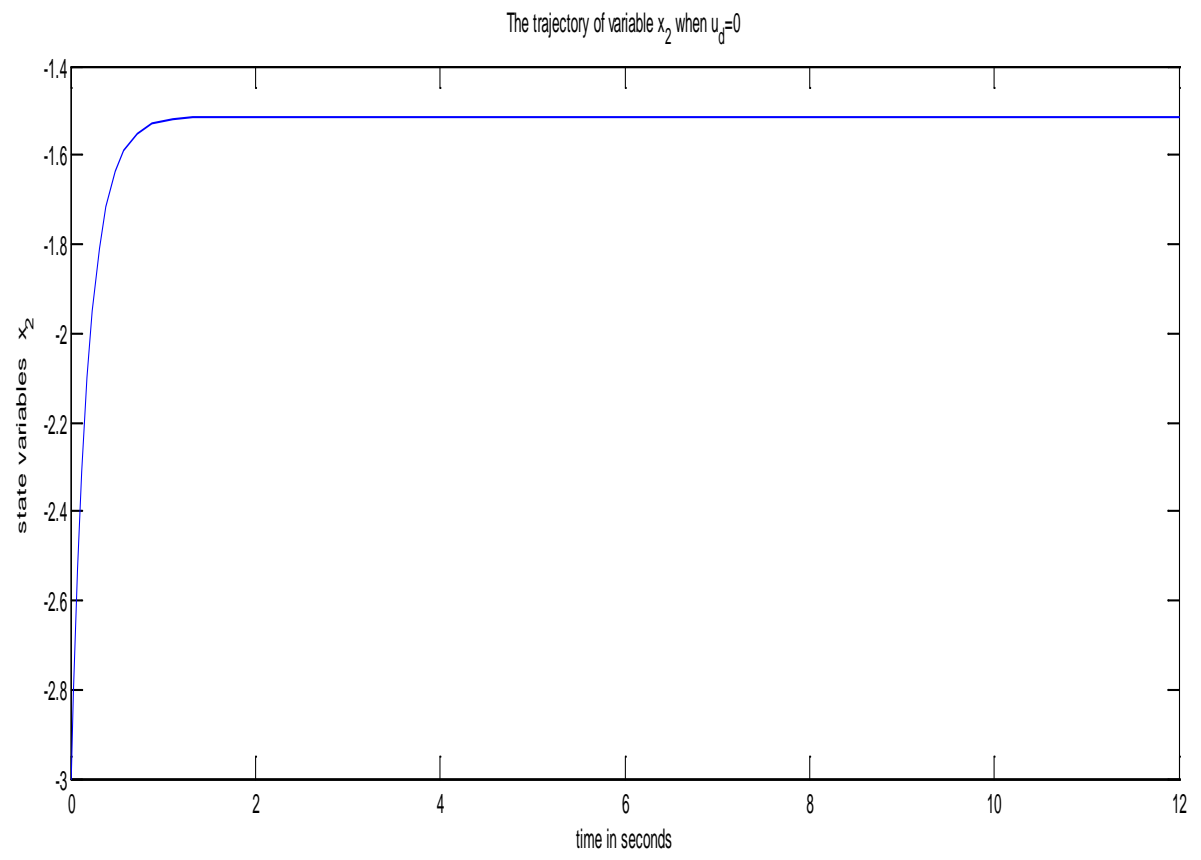

Figure 3.4c: Variable $X_{3}$ for $\eta=0$.

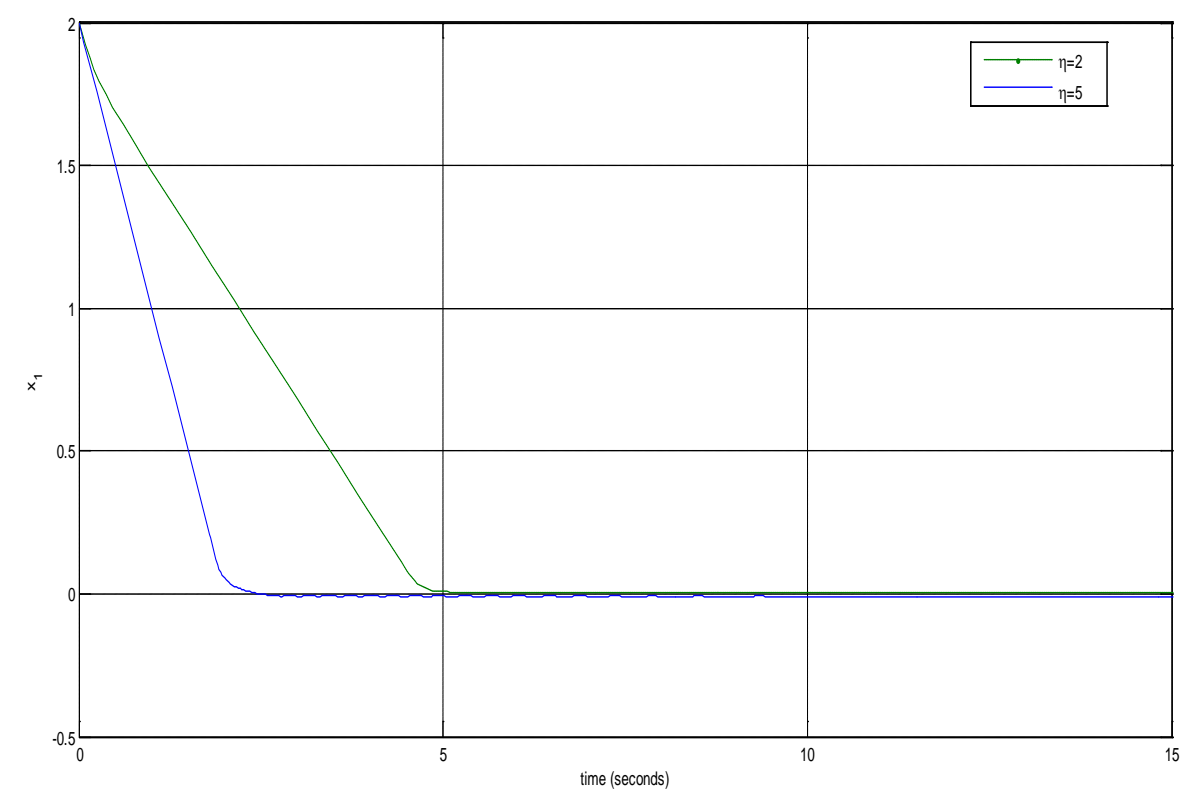

Figure 3.5a: The hyperplane for $\eta=2,5$ 


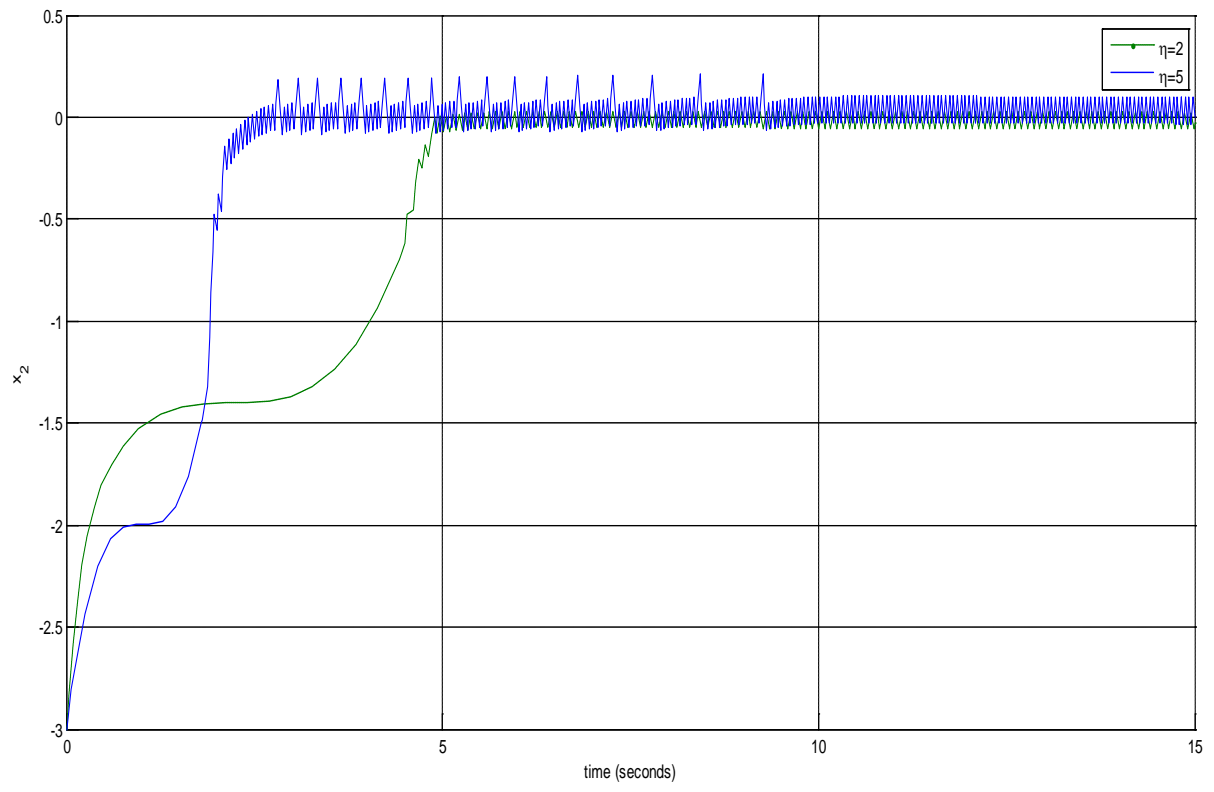

Figure 3.5b: Variable $x_{1}$ for $\eta=2,5$

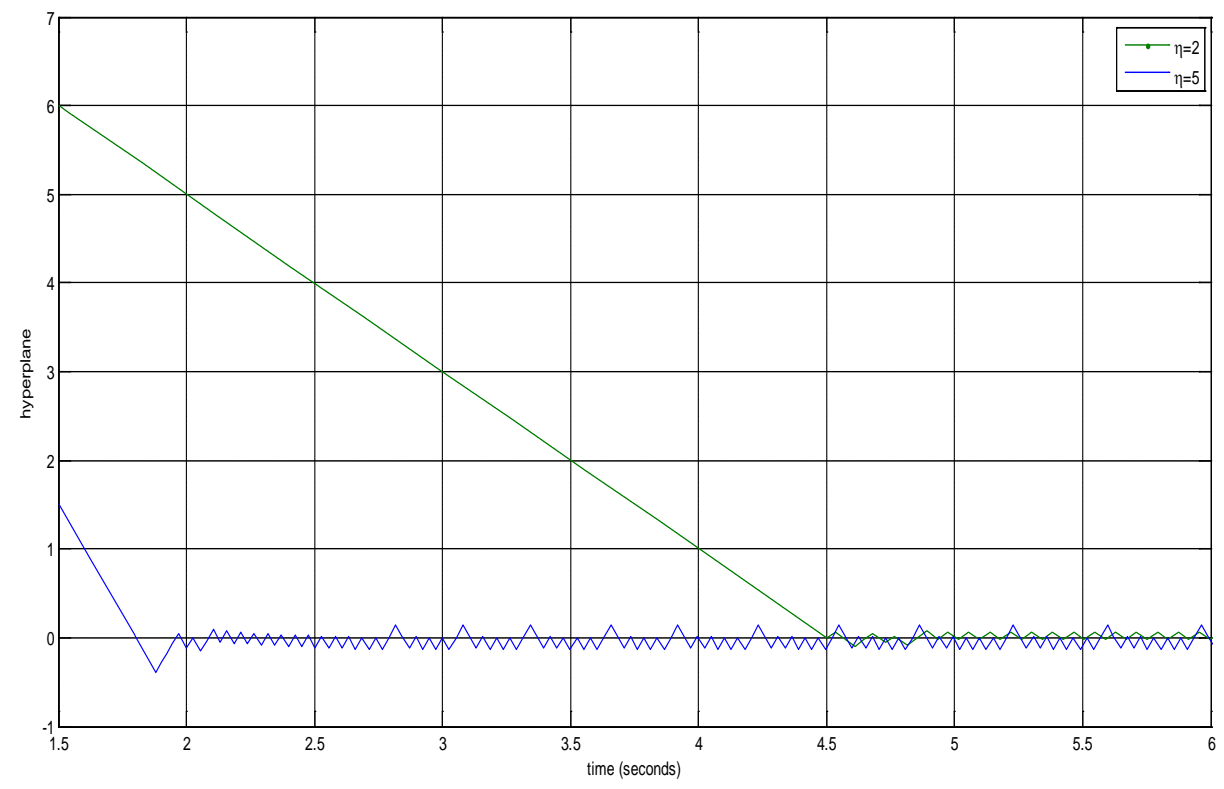

Figure 3.5c: Variable $x_{2}$ for $\eta=2,5$ 

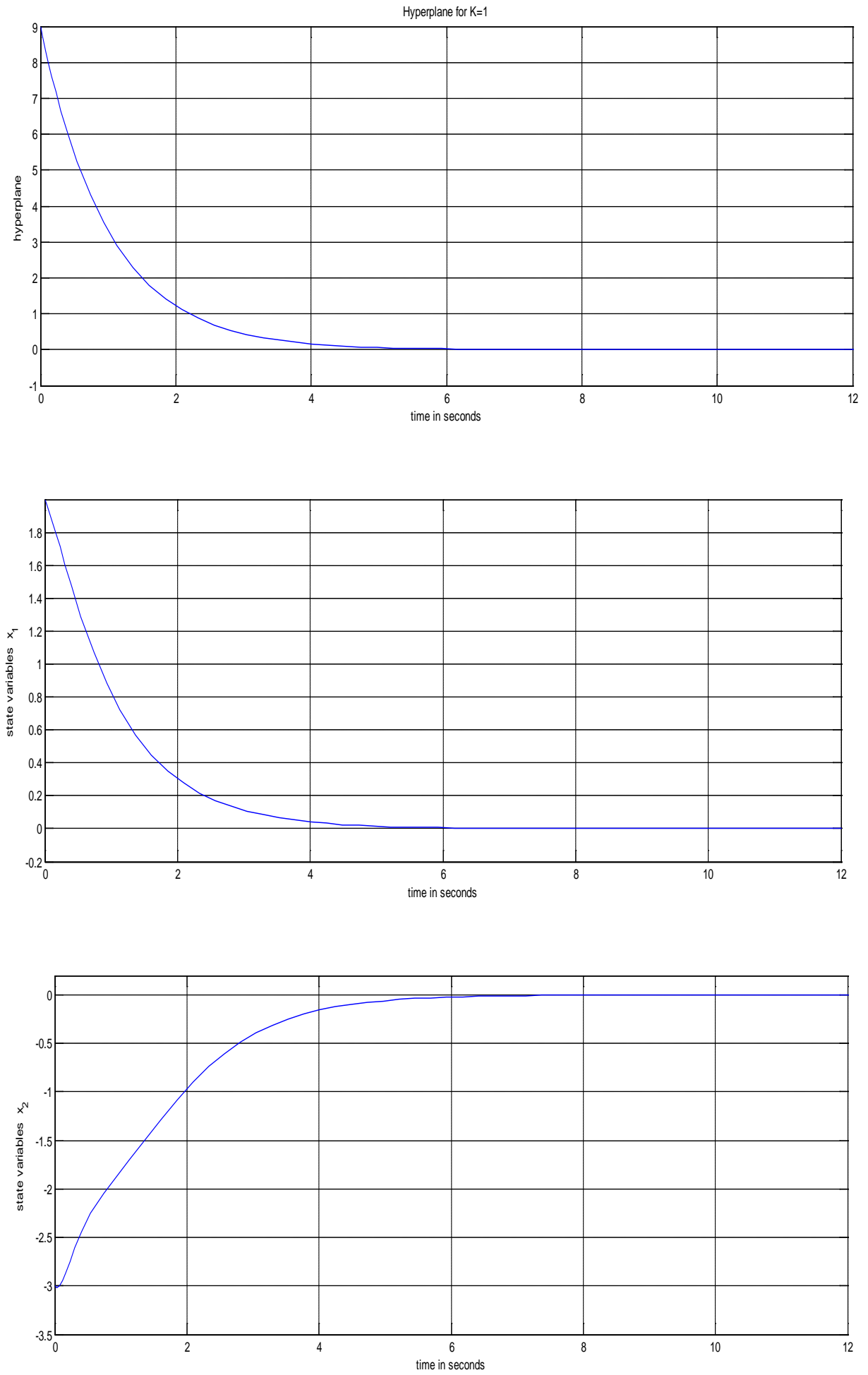

Figure 3.6: System response when the control law synthesized by using synergetic control approach is employed. The value of $k$ is taken to be 1 . 

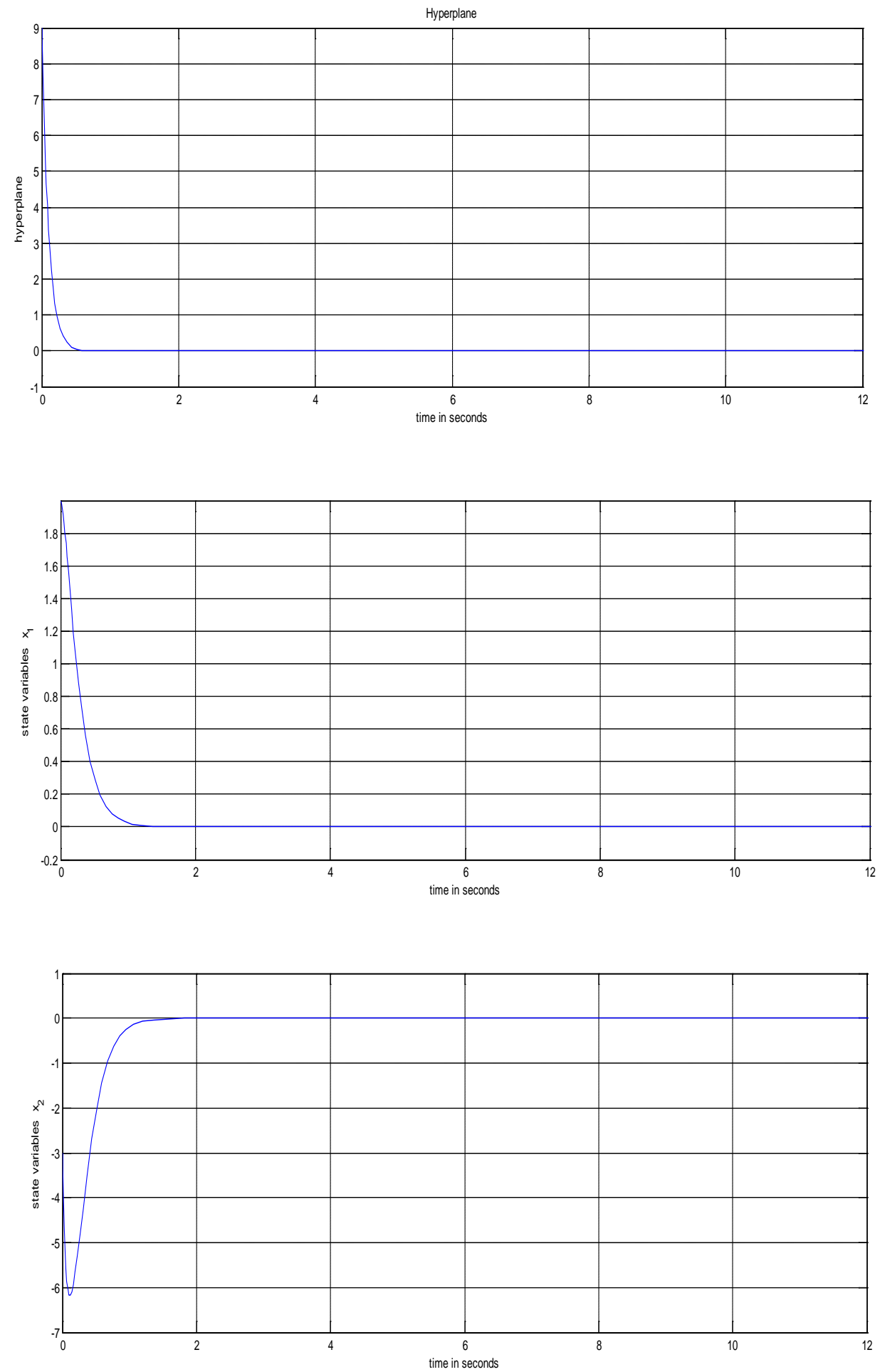

Figure 3.7: System response when the control law synthesized by using synergetic control approach is employed. The value of $K$ is taken to be 0.1 . 
reach it. In order to bring or force them onto the surface, the discontinuous part of the controller has to be present, i.e. $\eta>0$. Figures 3.5 shows the system's trajectories and the hyperplane for $\eta=2$, and 5 .

We can see from Figure 3.5 that increasing the value $\eta$ will cause the trajectories of the system to reach the surface as quickly as possible. But this will cause the chattering effect to be more pronounced. The results of the simulation when the controller expressed by (3.29) is used to stabilize the system are depicted in Figures 3.6 and 3.7 for $K=0$ and $K=0.1$ respectively. It can be seen from these Figures that chattering effect is completely removed when this controller is employed.

\subsection{Power System Problem Formulation}

In general the complete differential-algebraic model of any power system can be written as [3]:

$$
\begin{aligned}
& \dot{x}=f(x, V) \\
& Y V=I(x, V)
\end{aligned}
$$

where the parameters are represented as:

- $\quad x$-state vector of power system model

- $\quad V$ - bus voltage of the system

- $\quad I$-current injection vector into the system

- $\quad Y$ - admittance matrix, including constant impedance loads and the modification due to faults in the system

Both functions $f(x, V)$ and $I(x, V)$ are nonlinear and their values can be obtained if the operating condition $(x, V)$ is given. The initial values of (3.30) and (3.31) are 0 and $I\left(x_{0}, V_{0}\right)$ respectively. A multimachine power system consists of several synchronous generators connected through transmission lines. A decentralized damping controller is to be designed for this system. To design a decentralized controller, each machine needs to be modeled as a decoupled subsystem. The 
decoupling is obtained by estimating the effect of the rest of the system on this particular machine with a quadratic function of the active power generated by it. Throughout this study, each generator or subsystem is modeled by a third-order single axis model.

\subsection{1 ith-machine model}

The dynamical model of the $i^{\text {th }}$ generator with excitation control is given as $[39,40]$ :

Mechanical Equations:

$\dot{\delta}_{i}(t)=\omega_{o}\left(\omega_{i}(t)-1\right)$

$\dot{\omega}(t)=-\frac{D_{i}}{2 H_{i}}\left(\omega_{i}(t)-1\right)-\frac{1}{2 H_{i}}\left(P_{\text {mio }}-P_{e i}(t)\right)$

Generator Electrical Dynamic:

$\dot{E}_{q i}^{\prime}=\frac{1}{T_{d o i}^{\prime}}\left(E_{f d i}(t)-\left(x_{d i}-x_{d i}^{\prime}\right) I_{d i}-E_{q i}^{\prime}\right) I_{q i}$

Electrical Equations:

$E_{f d i}(t)=k_{c i} u_{f i}(t)$

$P_{e i}(t)=\sum_{j=1}^{n} E_{q i}^{\prime} E_{q j}^{\prime} B_{i j} \sin \left(\delta_{i}-\delta_{j}\right)$

$Q_{e i}(t)=-\sum_{j=1}^{n} E_{q i}^{\prime} E_{q j}^{\prime} B_{i j} \cos \left(\delta_{i}-\delta_{j}\right)$

$I_{d i}(t)=\sum_{j=1}^{n} E_{q j}^{\prime} B_{i j} \cos \left(\delta_{i}-\delta_{j}\right)$

$I_{q i}(t)=\sum_{j=1}^{n} E_{q j}^{\prime} B_{i j} \sin \left(\delta_{i}-\delta_{j}\right)$

Synchronous generator and transmission line variables are given in Table 3.1. 
Let $\delta_{\text {io }}, \omega_{\text {io }}=1, \mathrm{P}_{\text {eio }}$ be the desired values for the power angle, angular speed and the active power of the i-th generator, and denoting by:

$x_{1 i}=\delta_{i}-\delta_{i o}, x_{2 i}=\omega_{i}-1$, and $x_{3 i}=P_{e i}-P_{e i o}$

the dynamic model of the $i^{\text {th }}$ subsystem can be written as:

$$
\begin{aligned}
& \dot{x}_{1 i}=\omega_{0} x_{2 i} \\
& \dot{x}_{2 i}=-\frac{D_{i}}{2 H_{i}} x_{2 i}-\frac{1}{2 H_{i}} x_{3 i}
\end{aligned}
$$

Table 3.1: Synchronous parameter and transmission line variables

\begin{tabular}{|lll|}
\hline$\delta_{i}$ & --- & Rotor angle in radians \\
$\omega_{i}$ & --- & Speed of the generator in p.u. \\
$P_{m i o}$ & --- & Mechanical input power in p.u., assumed to be constant \\
$P_{e i}(t)$--- & Active electric power delivered by the generator in p.u. \\
$D_{i}$ & --- & p.u. damping constant \\
$H_{i}$ & --- & Inertia constant in seconds \\
$E_{q i}^{\prime}$ & --- & Equivalent EMF in the excitation coil in p.u. \\
$E_{f d i}$ & --- & Transient EMF behind the quadrature axis \\
$T_{d o i}^{\prime}$ & --- & Direct axis short circuit time constant \\
$Q_{e i}(t)--$ & Reactive power of the generator in p.u. \\
$I_{q i}, I_{d i}--$ & Quadrature and direct axis currents \\
$k_{c i}$ & --- & Gain of the exciter \\
$u_{f i}$ & --- & Controller input to the exciter \\
$x_{d i}, x_{d i}^{\prime}--$ & Direct axis reactance and transient reactance \\
$B_{i j}$ & Elements of susceptance matrix \\
\hline
\end{tabular}

By differentiating (3.36) and substituting (3.34), (3.35), (3.37) and (3.39) into derived derivative we have

$$
\dot{P}_{e i}=\dot{E}_{q i}^{\prime} I_{q i}+E_{q i}^{\prime} \dot{I}_{q i}
$$




$$
\begin{gathered}
\dot{P}_{e i}=\frac{1}{T_{d o i}^{\prime}}\left(k_{c i} I_{q i} u_{f i}-\left(x_{d i}-x_{d i}^{\prime}\right) I_{d i} I_{q i}-E_{q i}^{\prime} I_{q i}\right)+E_{q i}^{\prime} \sum_{j=1}^{n} \dot{E}_{q i}^{\prime} B_{i j} \sin \left(\delta_{i}-\delta_{j}\right)+ \\
E_{q i}^{\prime} \sum_{j=1}^{n} E_{q i}^{\prime} B_{i j} \cos \left(\delta_{i}-\delta_{j}\right)\left(\omega_{i}-\omega_{j}\right)
\end{gathered}
$$

This can be written as

$$
\begin{aligned}
\dot{P}_{e i}= & -\frac{1}{T_{d o i}^{\prime}} X_{3 i}+\frac{1}{T_{d o i}^{\prime}}\left(k_{c i} I_{q i} u_{f i}-\left(x_{d i}-x_{d i}^{\prime}\right) I_{d i} I_{q i}-P_{m i o}-T_{d o i}^{\prime} Q_{e i} X_{2 i}\right)+E_{q i}^{\prime} \sum_{j=1}^{n} \dot{E}_{q j}^{\prime} B_{i j} \sin \left(\delta_{i}-\delta_{j}\right) \\
& +E_{q i}^{\prime} \sum_{j=1}^{n} E_{q i}^{\prime} B_{i j} \cos \left(\delta_{i}-\delta_{j}\right)\left(-\omega_{j}\right)
\end{aligned}
$$

Let us define a new variable $v_{f i}$ as:

$v_{f i}=k_{c i} I_{q i} u_{f i}-\left(X_{d i}-x_{d i}^{\prime}\right) I_{d i} I_{q i}-P_{\text {mio }}-T_{d o i}^{\prime} Q_{e i} X_{2 i}$

and let us denote the coupling term between the $i^{\text {th }}$ subsystem and the rest of the system by:

$d_{i}=E_{q i}^{\prime} \sum_{j=1}^{n} \dot{E}_{q j}^{\prime} B_{i j} \sin \left(\delta_{i}-\delta_{j}\right)-E_{q i}^{\prime} \sum_{j=1}^{n} E_{q j}^{\prime} B_{i j} \cos \left(\delta_{i}-\delta_{j}\right)\left(\omega_{j}\right)$

By substituting Eqs. (3.44) and (3.45) into (3.43) we have

$$
\dot{x}_{3 i}=-\frac{1}{T_{d o i}^{\prime}} X_{3 i}+\frac{1}{T_{d o i}^{\prime}} v_{f i}+d_{i}
$$

Getting $u_{f i}$ from (3.44) is feasible since $I_{q i} \neq 0$ in the normal working region of a generator. Eq. (3.42) is obtained with the assumption that $P_{\text {mio }}=P_{\text {eio }}$. It includes information pertaining to the entire system. It is being considered as an external disturbance to the subsystem and it is modeled as a polynomial function of the active power generated by this subsystem, i.e. $d_{i} \approx c_{1 i} x_{3 i}+c_{2 i} x_{3 i}^{2}$, where $c_{1 i}$ and $c_{2 i}$ are unknown constants to be determined using adaptation law $[24,41]$. 


\subsubsection{Controller Design}

In this section, a decentralized synergetic controller is designed. Given a subsystem described by Eqs. (3.40), (3.41) and (3.45), a macro-variable for the ith generator is defined using the procedure of Slotine and $\mathrm{Li}[17]$ as:

$\varphi_{i}=\left(\frac{d^{2}}{d t^{2}}+\alpha_{i} \frac{d}{d t}+\alpha_{i}^{2}\right) x_{1 i}$

For the manifold that will evolve from Eq. (3.45) to be stable, $\alpha_{i}>0$.Substituting (3.40) and (3.41) into (3.46), we can re-express the macro-variable as:

$\varphi_{i}=\omega_{o}\left(\alpha_{i}-\frac{D_{i}}{2 H_{i}}\right) x_{2 i}-\frac{\omega_{o}}{2 H_{i}} x_{3 i}+\alpha_{i}^{2} x_{1 i}$

The dynamic of the evolution of the macro-variable for the ith subsystem is given as:

$$
K_{i} \dot{\varphi}_{i}+\varphi_{i}=0
$$

By substituting Eq. (3.47) and its derivative into Eq. (3.48) we have

$$
\begin{aligned}
& \omega_{o}\left(\alpha_{i}-\frac{D_{i}}{2 H_{i}}\right) \dot{X}_{2 i}-\frac{\omega_{o}}{2 H_{i}} \dot{X}_{3 i}+\alpha_{i}^{2} \dot{X}_{1 i}=-\frac{1}{K_{i}}\left[\omega_{o} \dot{X}_{2 i}+\omega_{o} X_{2 i}+\alpha_{i}^{2} x_{1 i}\right] \\
& -\frac{\omega_{o}}{2 H_{i}} \dot{X}_{3 i}=-\left\{\omega_{0}\left(\alpha_{i}-\frac{D_{i}}{2 H_{i}}\right) \dot{X}_{2 i}+\omega_{0} \alpha_{i}^{2} x_{2 i}+\frac{1}{K_{i}}\left(\omega_{o} \dot{x}_{2 i}+\omega_{o} \alpha_{i} X_{2 i}+\alpha_{i}^{2} x_{1 i}\right)\right\}
\end{aligned}
$$

this gives

$$
\dot{x}_{3 i}=2 H_{i}\left(\alpha_{i}-\frac{D_{i}}{2 H_{i}}+\frac{1}{K_{i}}\right) \dot{X}_{2 i}+2 H_{i}\left(\alpha_{i}^{2}+\frac{\alpha_{i}}{K_{i}}\right) x_{2 i}+\frac{2 H_{i} \alpha_{i}^{2}}{\omega_{0} K_{i}} x_{1 i}
$$

By substituting Eq. (3.45) into (3.51) we have 
$v_{f i}+T_{d o i}^{\prime} d_{i}=x_{3 i}+2 H_{i} T_{d o i}^{\prime}\left(\alpha_{i}-\frac{D_{i}}{2 H_{i}}+\frac{1}{K_{i}}\right) \dot{x}_{2 i}+2 H_{i} T_{d o i}^{\prime}\left(\alpha_{i}^{2}+\frac{\alpha_{i}}{K_{i}}\right) x_{2 i}+\frac{2 H_{i} T_{d o i}^{\prime} \alpha_{i}^{2}}{\omega_{0} K_{i}} x_{1 i}$

Let us define $\hat{d}=\hat{c}_{1 i} x_{3 i}+\hat{c}_{2 i} x_{3 i}^{2}$, where $\hat{c}_{1 i}$ and $\hat{c}_{2 i}$ are the estimate of $c_{1 i}$ and $c_{2 i}$. The objective is to approximate the coupling term using adaption law and let us define a Lyapunov function as:

$\bar{V}=\frac{1}{2} \varphi_{i}^{2}+\frac{1}{2}\left(c_{1 i}-\hat{c}_{1 i}\right)^{2} \Gamma_{1 i}^{-1}+\frac{1}{2}\left(c_{2 i}-\hat{c}_{2 i}\right)^{2} \Gamma_{2 i}^{-1}$

where $\Gamma_{1 i}$ and $\Gamma_{2 i}$ are adaptive gains. By differentiating (3.53) we have

$\dot{\bar{V}}=\varphi_{i} \dot{\varphi}_{i}+\left(c_{1 i}-\hat{c}_{1 i}\right) \Gamma_{1 i}^{-1} \dot{\hat{c}}+\left(c_{2 i}-\hat{c}_{2 i}\right) \Gamma_{2 i}^{-1} \dot{\hat{c}}_{2 i}$

Let define

$\dot{\hat{c}}_{1 i}=\Gamma_{1 i} \varphi_{i} x_{3 i}$

$\dot{\hat{c}}_{2 i}=\Gamma_{2 i} \varphi_{i} x_{3 i}^{2}$

By substituting (3.45), (3.55) and (3.56) into (3.54) and solving for $v_{f i}$ to make (3.54) negative, we can obtain

$$
\begin{aligned}
v_{f i}= & x_{3 i}+2 H_{i} T_{d o i}^{\prime}\left(\alpha_{i}-\frac{D_{i}}{2 H_{i}}+\frac{1}{K_{i}}\right) \dot{x}_{2 i}+2 H_{i} T_{d o i}^{\prime}\left(\alpha_{i}^{2}+\frac{\alpha_{i}}{K_{i}}\right) x_{2 i}+\frac{2 H_{i} T_{d o i}^{\prime} \alpha_{i}^{2}}{\omega_{o} K_{i}} x_{1 i} \\
& -T_{d o i}^{\prime} \hat{c}_{1 i} x_{3 i}-T_{d o i}^{\prime} \hat{c}_{2 i} x_{3 i}^{2}
\end{aligned}
$$

Substituting (3.51) into (3.35) reduces $\dot{\bar{V}}$ into

$$
\dot{\bar{V}}=-\frac{1}{K_{i}} \varphi_{i}^{2}<0
$$


Hence, the system is guaranteed to be stable. $v_{f i}$ can be considered to consist of two terms: The first term is to move and restrict the variables of the ith subsystem to the manifold of interest, i.e. $\varphi=0$; while the second term is to cancel out the coupling effect on the ith subsystem. By substituting (3.43) into (3.57), the exciter input can be obtained as:

$$
\begin{aligned}
u_{f i}= & \frac{1}{k_{c i} I_{q i}}\left\{P_{m i o}+\left(x_{d i}-x_{d i}^{\prime}\right) I_{d i} I_{q i}+T_{d o i}^{\prime} Q_{e i} x_{2 i}-\left(1-T_{d o i}^{\prime} \hat{c}_{1 i}\right) x_{3 i}-T_{d o i}^{\prime} \hat{c}_{2 i} x_{3 i}^{2}+\frac{2 H_{i} T_{d o i}^{\prime} \alpha_{i}^{2}}{\omega_{o} K_{i}} x_{1 i}\right. \\
& +2 H_{i} T_{d o i}^{\prime}\left(\alpha_{i}-\frac{D_{i}}{2 H_{i}}+\frac{1}{K_{i}}\right) \dot{x}_{2 i}+2 H_{i} T_{d o i}^{\prime}\left(\alpha_{i}^{2}+\frac{\alpha_{i}}{K_{i}}\right) x_{2 i}
\end{aligned}
$$

By appropriate choice of the controller gains, $\alpha_{i}, \Gamma_{1 i}, \Gamma_{2 i} K_{i}$ and $k_{c i}$, the power angle oscillations can be damped effectively. From the example illustrated above, it was shown that the performance of the system depends on the controller parameters. To obtain optimal results the controller's gains are tuned using Particle Swarm Optimization (PSO) algorithm. This method is discussed in detail in the next section.

\subsection{Particle Swarm Optimization}

Particle Swarm Optimization is an evolutionary algorithm developed by James Kennedy and Eberhart [6] as a technique used to solving nonlinear continuous-variables optimization problems. It is a technique inspired by social behavior and movement of birds, insects or fish. The search algorithm is based on cooperation and contention among the population members. The goal is to obtain optimal regions of a complex search space through interaction of individuals in a population of particles. The algorithm works by first creating a 'population' of random solutions (also known as candidate solutions or particles). Each candidate solution of population has an adaptable velocity, according to which it moves in search space. Furthermore, each candidate has the capacity to remember the best position (or fitness) of the search space it has ever visited. Its movement is a conglomeration of acceleration toward its best previously visited position. The other best value tracked by PSO is called 'global best value', which is the best value or fitness found so far by any of candidate in the neighborhood of the candidate. The major concept is to alter the position and velocity of each candidate toward its local and global best locations at each time step. Consequently, 
after number of time steps the particle among populations are found to have gathered around one or more of the optima and tends to locate the global optima among all.

Unlike the other evolutionary algorithms like Genetic Algorithm (GA), PSO has a well-balanced and flexible mechanism to improve the ability to locate the best local and global positions [43]. Some of the advantages of PSO over other optimization techniques are [44]:

- The quality of the solution obtained from this technique is independent of the initial population

- It has more effective memory capability (local and neighboring best)

- It can overcome the untimely convergence problem and improve the search capability

- It has less parameters to adjust

- It is more flexible, robust and easy to implement

- It is less vulnerable to getting trapped on local minimal

Throughout the course of this study, PSO is utilized to electric power system to obtain the optimal value of the synergetic controller parameters. Some definition of the terminologies of PSO is given below:

Particle $\Omega_{i}(t)$ : A candidate solution (controllers' parameters) at iteration $t$. The size of each particle is the sum of the controllers' parameters. For the synergetic controller designed in the previous section, the number of parameters of the $i^{\text {th }}$ controller is 5 . Thus for a system of $n$ number of controllers, the size of each particle $(p)$ is $5 n$.

Population: A set of $m$ particles $\left\{\Omega_{1}(t) ; \Omega_{2}(t) ; \ldots \Omega_{m}(t)\right\}$, where $m$ is the number of candidate solutions.

Objective function $J_{i}(t)$ : function use to determine the fitness of $i^{\text {th }}$ controller at $t^{\text {th }}$ iteration.

Individual best $\Omega_{i}^{*}(t)$ : Also known as local best. It is the best value of fitness that this particle has achieved up to $t^{\text {th }}$ iteration.

$$
\begin{aligned}
& \Omega_{i}^{*}(t)=\left\{\Omega_{i}(t): J_{i}\left(\Omega_{i}^{*}(t)\right) \leq J_{i}\left(\Omega_{i}(\tau)\right), \quad \tau \leq t\right. \\
& J_{i}^{*}(t)=J_{i}\left(\Omega_{i}^{*}(t)\right)
\end{aligned}
$$


Global best $\Omega^{* *}(t)$ : The best position (or fitness) for all particles among all individual local best positions.

$$
\Omega^{* *}(t)=\left\{\Omega_{i}^{*}(t): J\left(\Omega^{* *}(t)\right) \leq J_{i}\left(\Omega_{i}^{*}(t)\right), i=1, \ldots, n\right\}
$$

The steps of the PSO technique are delineated below:

- Initialization

1) Set the time counter $t$, performance evaluation counter $c$, and their maximum values $t_{\max }, C_{\max }$

2) Generate arbitrarily $m$ particles and velocity for each of these particles. These values are generated from the following given information: the size of each particle $p$, the population size $m$, the admissible range for controller parameters $\phi_{j, j j}^{\min }, \phi_{j, j j}^{\max }$

$$
\phi_{j, j j}^{\min } \leq \phi_{j, j j} \leq \phi_{j, j j}^{\max } \quad j=1,2, \cdots, p, \quad j j=1,2, \cdots, m
$$

and the admissible range of velocity

$$
-v_{j, j j}^{\max } \leq v_{j, j j} \leq v_{j, j j}^{\max }
$$

Where

$v_{j, j j}^{\max }=\frac{\phi_{j, j j}^{\max }-\phi_{j, j j}^{\min }}{\mu}$

and $\mu$ is the number of interval in $j j^{\text {th }}$ dimension.

3) For each particle, determine the fitness, then search for the best values among all the fitness values and set this value as the global best fitness $J^{* *}(t)$ and the particle that gives this fitness value as the global best position $\Omega^{* *}(t)$ i.e. $J_{i}^{*}(t)=J_{i}(t), \Omega_{i}^{*}(t)=\Omega_{i}(t)$ and $J^{* *}(t, c)=\min \left[J_{1}^{*}(t) \ldots J_{n}^{*}(t)\right]$ 
4) Set $t=t+1, c=c+1$

- Update the velocity using the following equation

$$
\begin{aligned}
& v_{j, j j}(t)=\Psi\left(v_{j, j j}(t-1)\right)+c_{1} r_{1}\left(\phi_{j, j j}^{*}(t-1)-\phi_{j, j j}(t-1)\right)+c_{2} r_{2}\left(\phi_{j, j j}^{* *}(t-1)-\phi_{j, j j}(t-1)\right) \\
& \Psi=\frac{2}{\left|2-\chi-\sqrt{\chi^{2}-4 \chi}\right|}
\end{aligned}
$$

where

$$
\chi=c_{1}+c_{2}, \chi>4, \phi_{j, j j}^{*}(t) \text { and } \phi_{j, j j}^{* *}(t) \text { are local best and global best parameters of controller. }
$$

- Updating the position

As a result of velocities update, each particle changes its position according to the following equation

$\phi_{j, j j}(t)=\phi_{j, j j}(t-1)+v_{j, j j}(t)$

Update the position $\Omega_{i}(t)=\left[\phi_{j, 1}(t), \phi_{j, 2}(t), \cdots \phi_{j, m}(t)\right]$ for $j=1,2, \cdots p$

- Individual and global best update

Using the updated position, each particle evaluates its objective function and new individual best $J_{i}^{*}(t)$ and associated $\Omega_{i}^{*}$ for each $i$ are determined from (3.60) and (3.61). Eq. (3.62) is used to determine the global best i.e. to find $J^{* *}(t, c)=J\left(\Omega^{* *}(t, c)\right)$. If

$J^{* *}(t, c) \leq J^{* *}(t-1, c-1)$

then the fitness has improved, and the parameters are updated, set $c=c+1$ and proceed to the next step. Otherwise, update $J^{* *}(t, c), \Omega^{* *}(t)$ and set $c=0$. Then proceed to the next step

- Stopping criteria

The search process will terminate if one of the following conditions is satisfied: 
1) If the counter has attained its maximum number, $c=C_{\max }$ and the best global solution $J^{* *}(t, c)$ can no longer be improved. The optimal solution is considered to be the current $\Omega^{* *}(t)$

2) If the maximum allowable iteration is reached, $t=t_{\max }$.

\subsection{Case Studies}

\section{Single Machine Infinite Bus}

In this case study, a single machine infinite bus is considered. The model consists of a single generator connected to infinite bus via two parallel transmission lines [3]. Dynamic loads are connected at bus 2 . The parameters of the component of the system are specified in the Appendix. The study is presented to illustrate the potentiality of the proposed controller during and after contingency in the system. The results are compared with the traditional Power System Stabilizer (PSS). The schematic diagram of the system is depicted in Figure 3.8. The goal is to improve the system transient stability by designing excitation controller.

Supplementary control signal is used to add damping to the oscillations of the system. This signal is implemented via excitation control [45]. Three different control strategies are analyzed. These strategies are synergetic controller with excitation control, PSS with excitation control, and excitation control with no supplementary signal.

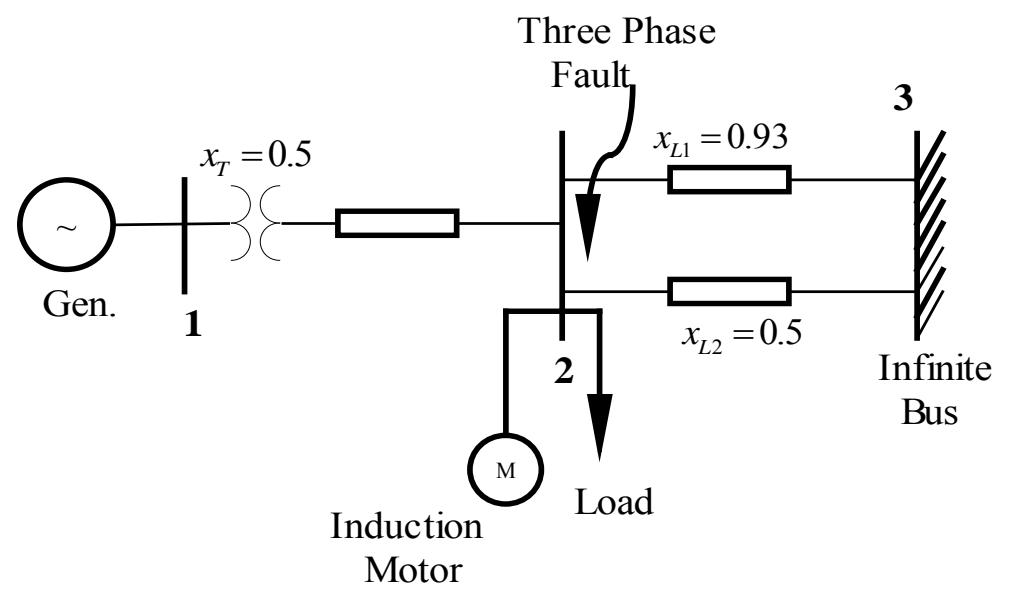

Figure 3.8: Single Machine Infinite Bus System 
The parameters of the synergetic controller are obtained by PSO technique. A three phase fault occurs on the first transmission line at time $t=0.2 \mathrm{~s}$. The fault is cleared at $t=0.27 \mathrm{~s}$ and the line is reconnected at $t=0.36 \mathrm{~s}$. The objective function used in the determination of the fitness of each particle for the PSO is defined as:

$$
J=\int_{0}^{t} k_{1}(\Delta \delta)^{2}+k_{2}(\Delta \omega)^{2}+k_{3}\left(\Delta V_{T}\right)^{2}
$$

Where $\Delta V_{T}$ is the deviation of the terminal voltage of the generator and $k_{1}, k_{2}$, and $k_{3}$ are weighing factors. After initializing the system and running the PSO algorithm, the optimum controller' parameters for the synergetic damping controller are given as:

$$
\lambda=10.3131, K=0.228, k_{c}=1
$$

Figures 3.9, 3.10 and 3.11 show comparison between the proposed controllers, PSS, and the simple exciter. The parameters for the exciter can be found in the appendix. PSS consists of two stage leadlag with a wash out stage (3.65).

$$
G(s)=K_{w} \frac{s T_{w}}{1+s T_{w}} \frac{\left(1+s T_{1}\right)^{2}}{\left(1+s T_{2}\right)^{2}}
$$

The gains of the PSS are tuned using PSO techniques and the result obtained is:

$$
G_{1}(s)=13.775 \frac{1.41 s}{1+1.41 s} \frac{(1+0.154 s)^{2}}{(1+0.033 s)^{2}}
$$

The proposed controller effectively dampens the oscillation and modulates the voltage. Better performance is achieved when compared to that of the PSS. The values of the function described in (3.70) are $0.116,0.1865$ and 2.276 for the system with synergetic controller, PSS, and with only simple exciter respectively. 
36

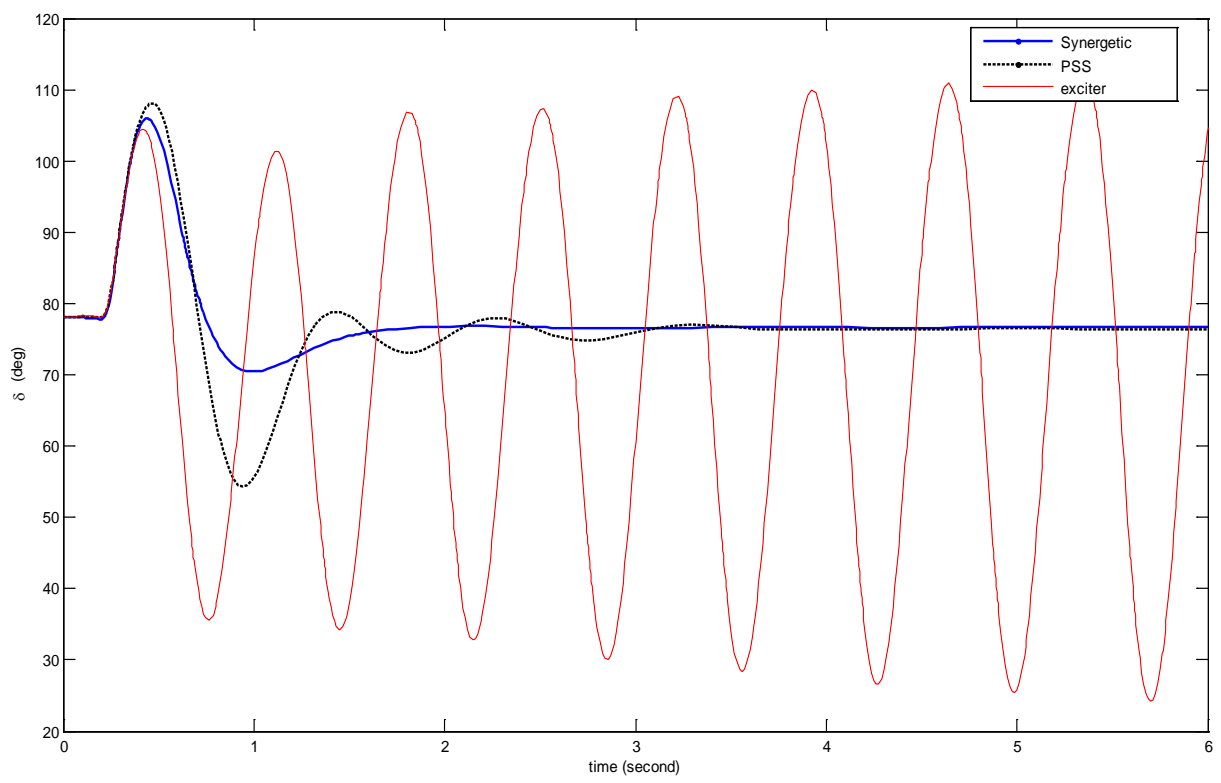

Figure 3.9: Power angle of the generator (SMIB System)

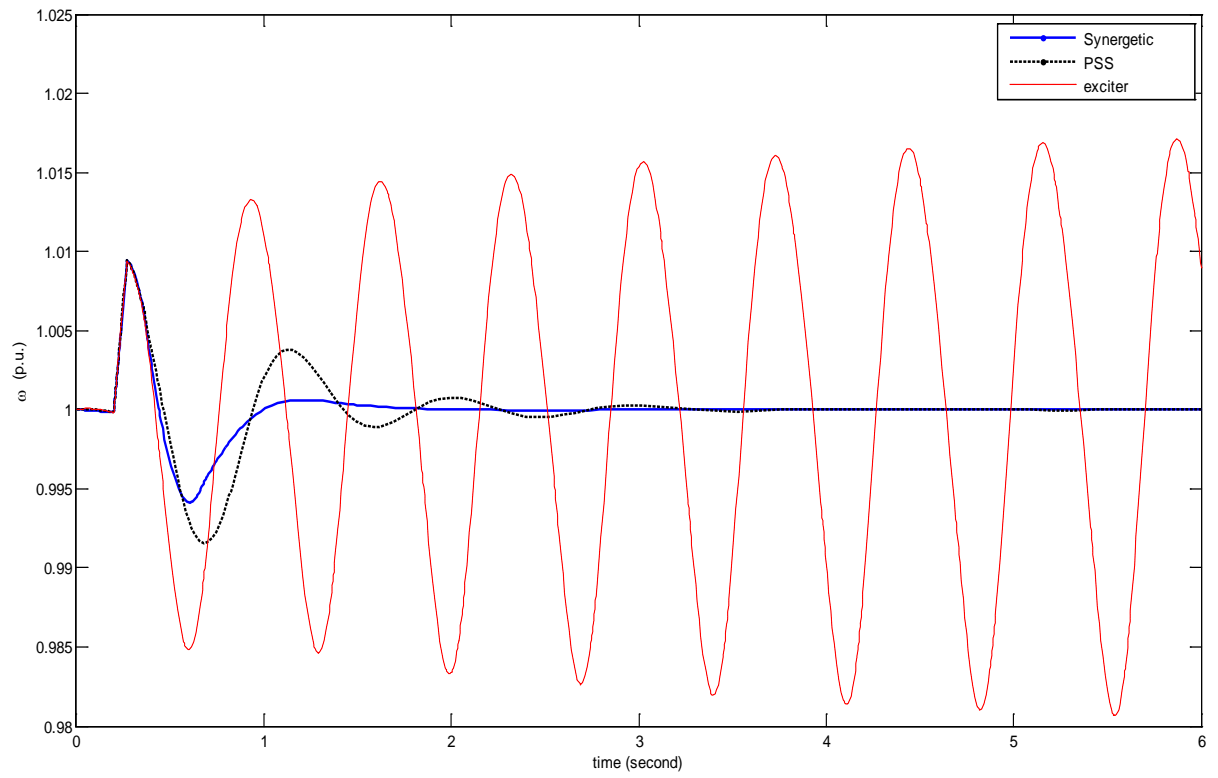

Figure 3.10: Angular speed of the generator (SMIB System.) 


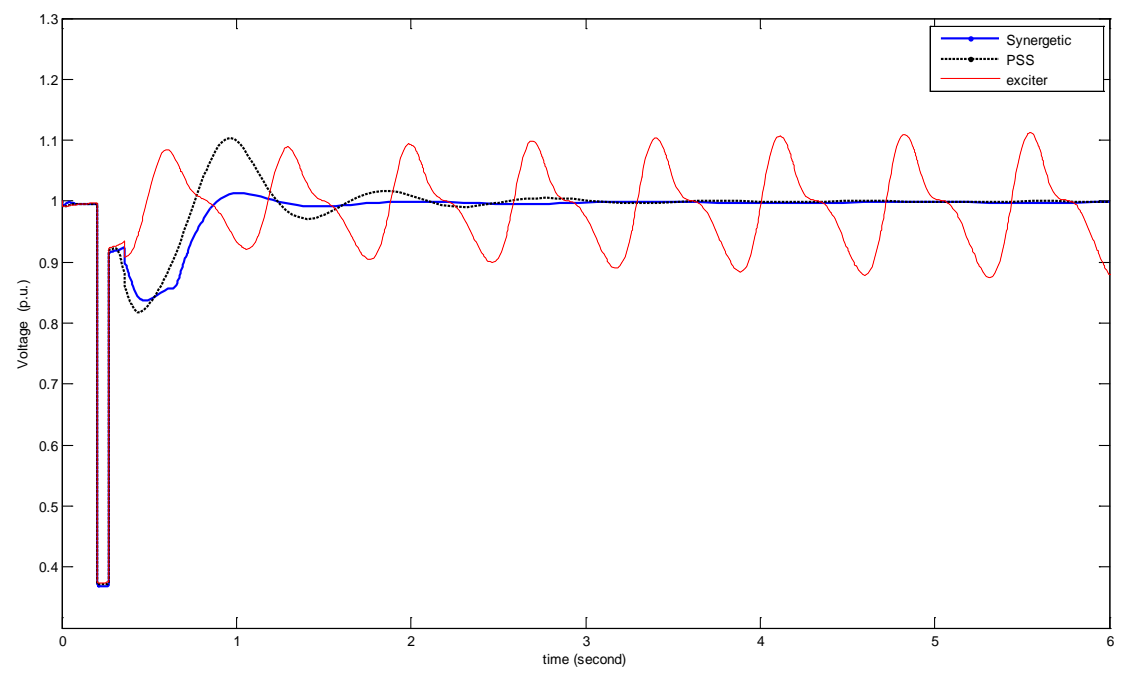

Figure 3.11: Terminal voltage of the generator (SMIB system).

\section{Three Machine Nine Bus Test System}

In this case study, the popular 9-bus 3-machines WSCC system is considered. Synergetic controllers are implemented as supplementary signals to exciters of the machines at buses 1, 2 and 3. An inductive load is added at bus 8 to test the performance of the controller to nonlinear loads. The line diagram of the system is depicted in Figure 3.13. The parameters of the exciters can be found in the Appendix.

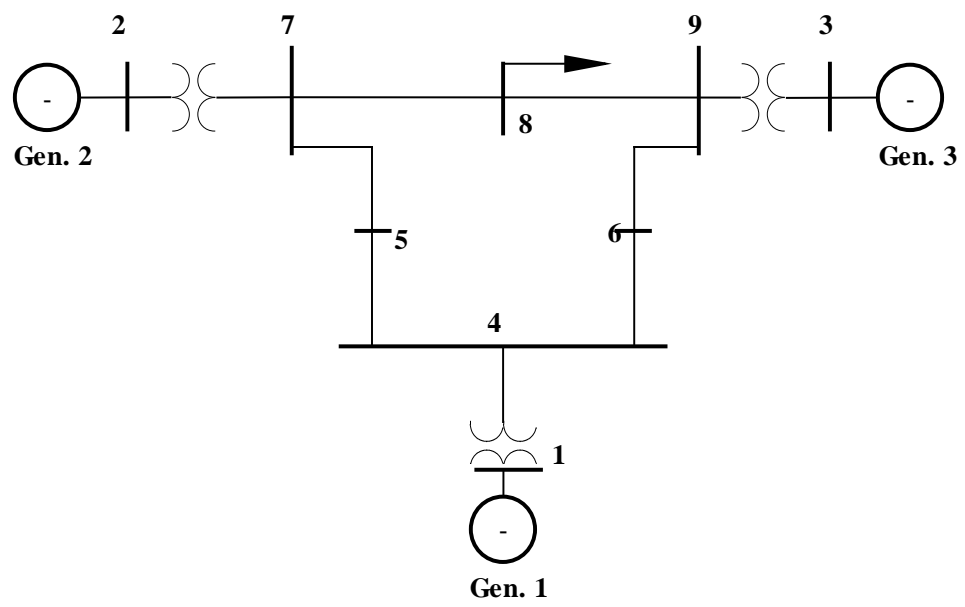

Figure 3.12: Three Machine Nine Bus Power System 
The system is tested for tolerance of a three phase fault on transmission line 6-9. A three phase fault occurred at 0.5 seconds. The fault is cleared after 20 milliseconds and the line is re-connected at 0.54 seconds. Figures 3.13-3.17 show comparison between the proposed controllers, and PSS. The gains of the PSS are tuned using PSO techniques and the result obtained is:

$$
\begin{aligned}
& G_{1}(s)=12.0963 \frac{5 s}{1+5 s} \frac{(1+0.1882 s)^{2}}{(1+0.05 s)^{2}} \\
& G_{2}(s)=8.225 \frac{5 s}{1+5 s} \frac{(1+0.201 s)^{2}}{(1+0.05 s)^{2}} \\
& G_{3}(s)=0.082 \frac{5 s}{1+5 s} \frac{(1+0.631 s)^{2}}{(1+0.05 s)^{2}}
\end{aligned}
$$

And the parameters of the synergetic damping controllers are obtained to be:

$$
\begin{aligned}
& \text { Gen.1: } \alpha_{1}=9.0 ; \Gamma_{11}=1 ; \Gamma_{21}=15.0 ; K_{1}=0.15 ; k_{c 1}=1.5 \\
& \text { Gen.2: } \alpha_{2}=9.0 ; \Gamma_{12}=4 ; \Gamma_{22}=18.0 ; K_{2}=0.015 ; k_{c 2}=1.5 \\
& \text { Gen.3: } \alpha_{3}=7.0 ; \Gamma_{13}=3 ; \Gamma_{23}=21.0 ; K_{3}=0.015 ; k_{c 3}=1.5
\end{aligned}
$$

The decentralized synergetic controllers dampen the rotor angle oscillations more effectively, and the generators are able to reach the synchronous speed faster when compared with the PSS performance (Figures 3.13 to 3.17). The objective function used in the determination of the fitness of each particle for the PSO for each of the generators is defined as:

$$
J_{i}=\int_{0}^{t}\left(k_{1} \Delta \delta_{i}^{2}+k_{2} \Delta \omega_{i}^{2}+k_{3} \Delta V_{T i}^{2}\right.
$$

And the value of this function for each of the generators is given in Table 3.2 below. 


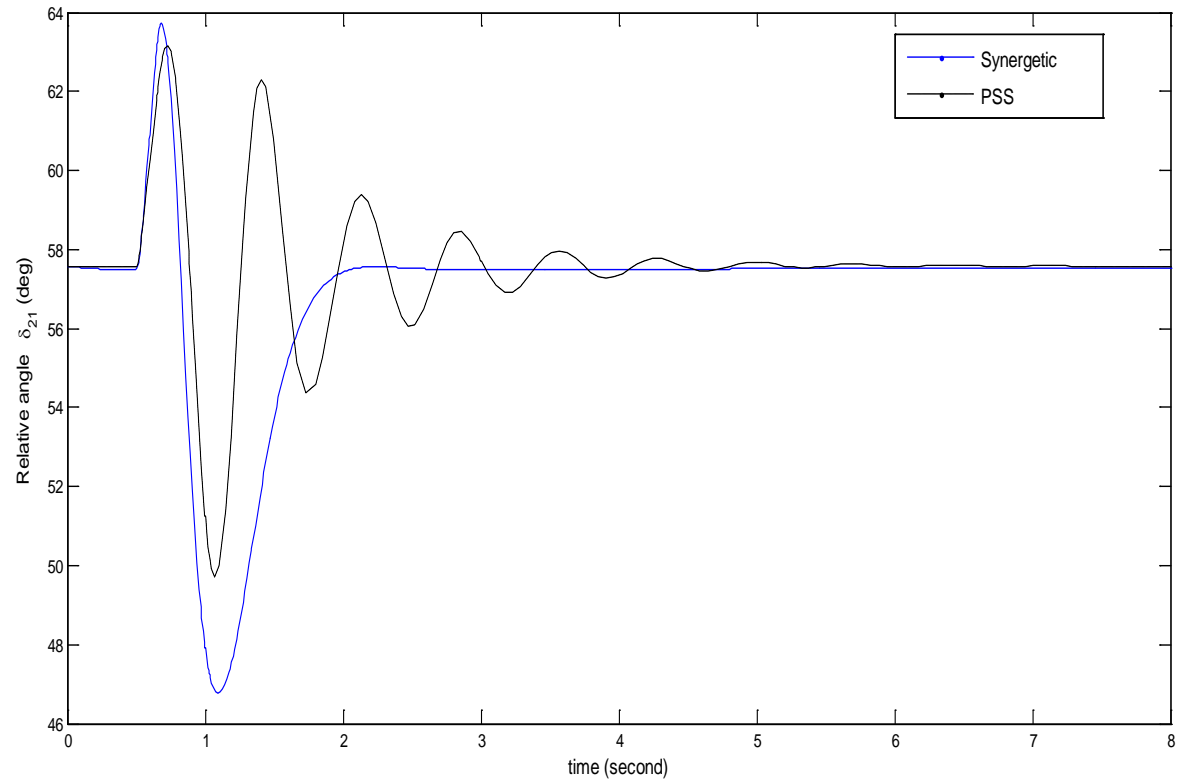

Figure 3.13: Relative rotor angle (degree) 2 - 1 (3-Machine Nine Bus System)

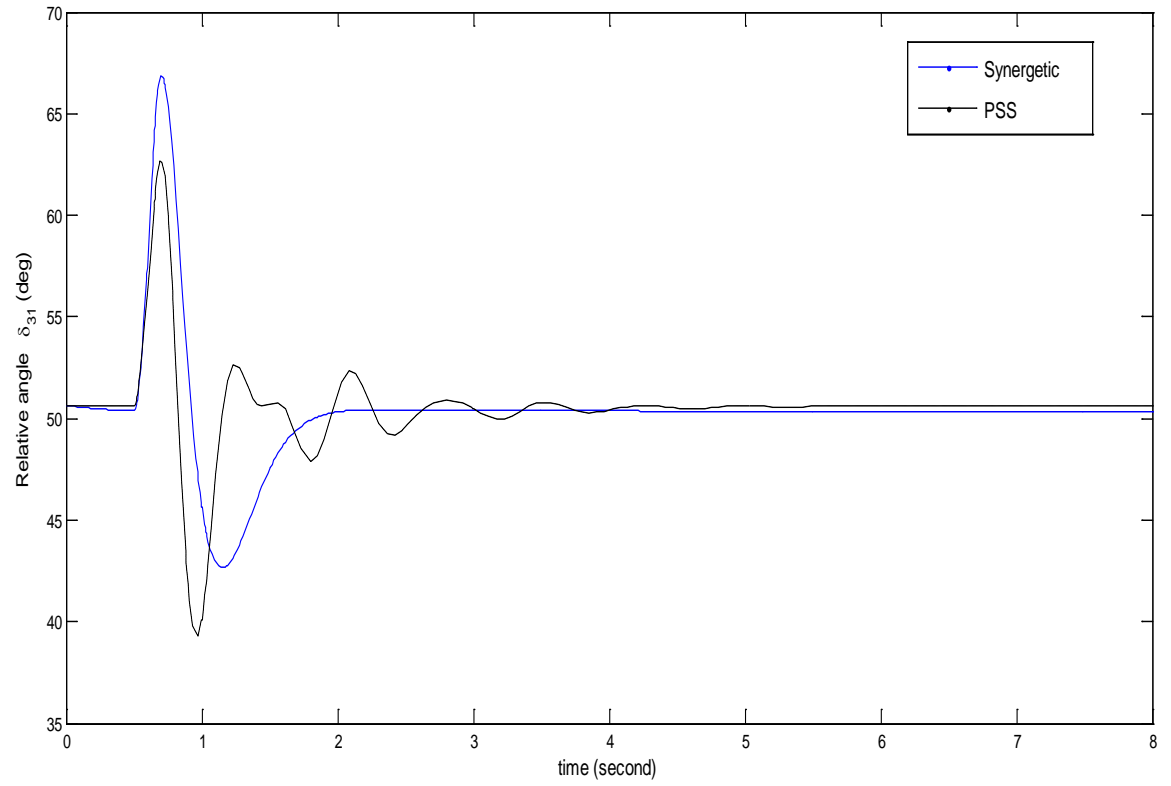

Figure 3.14: Relative rotor angle (degree) 3 - 1 (3-Machine Nine Bus System) 


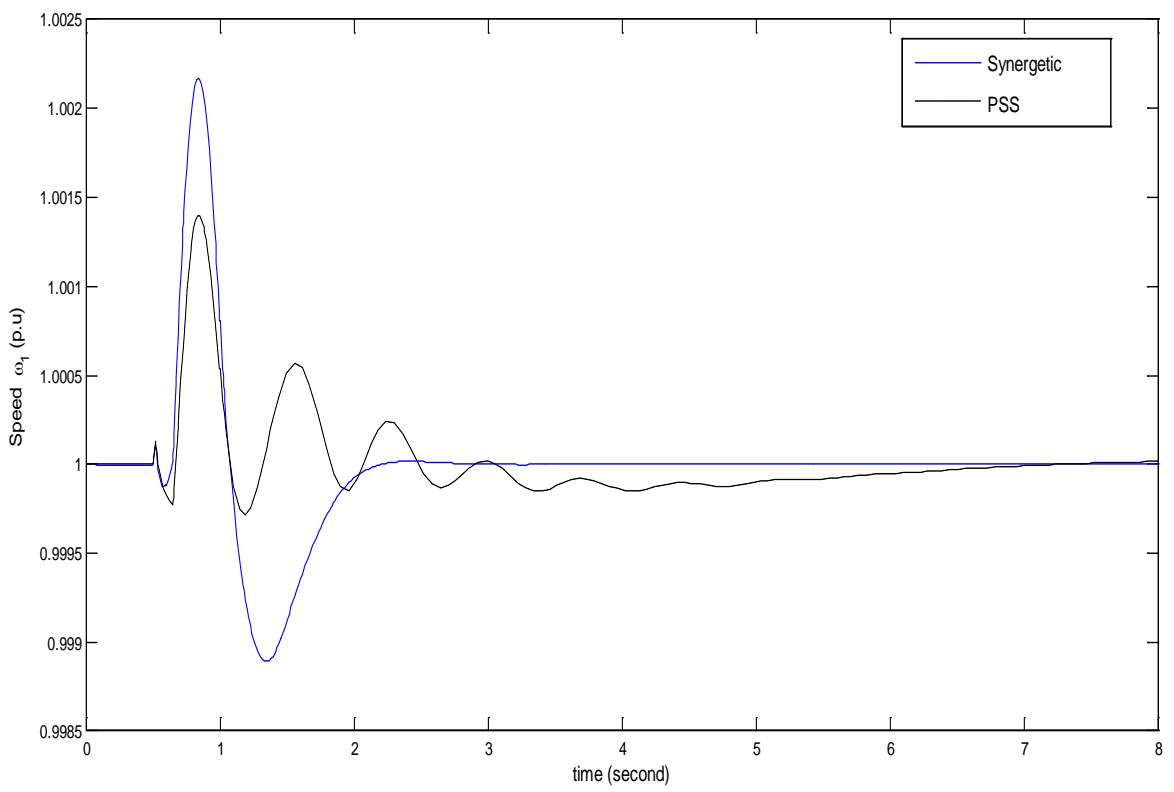

Figure3.15: Speed of generator 1. (3-Machine Nine Bus System)

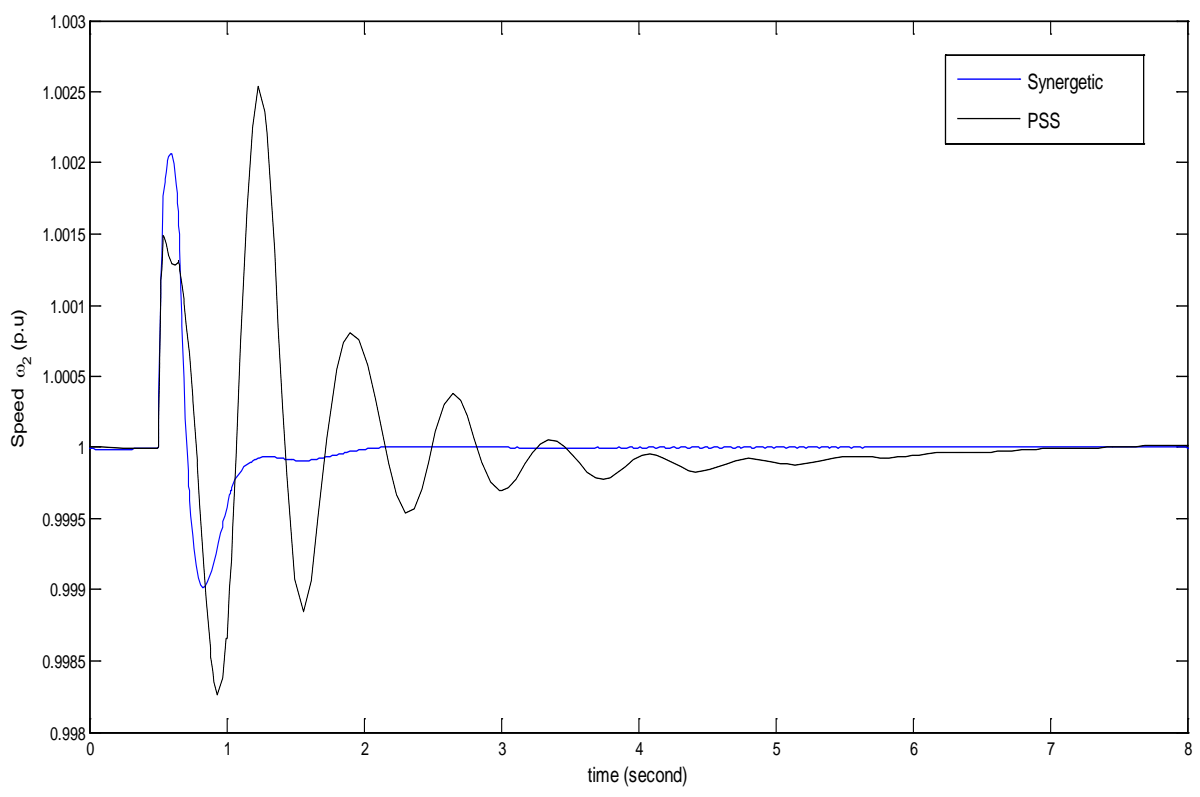

Figure 3.16: Speed of generator 3. (3-Machine Nine Bus System) 


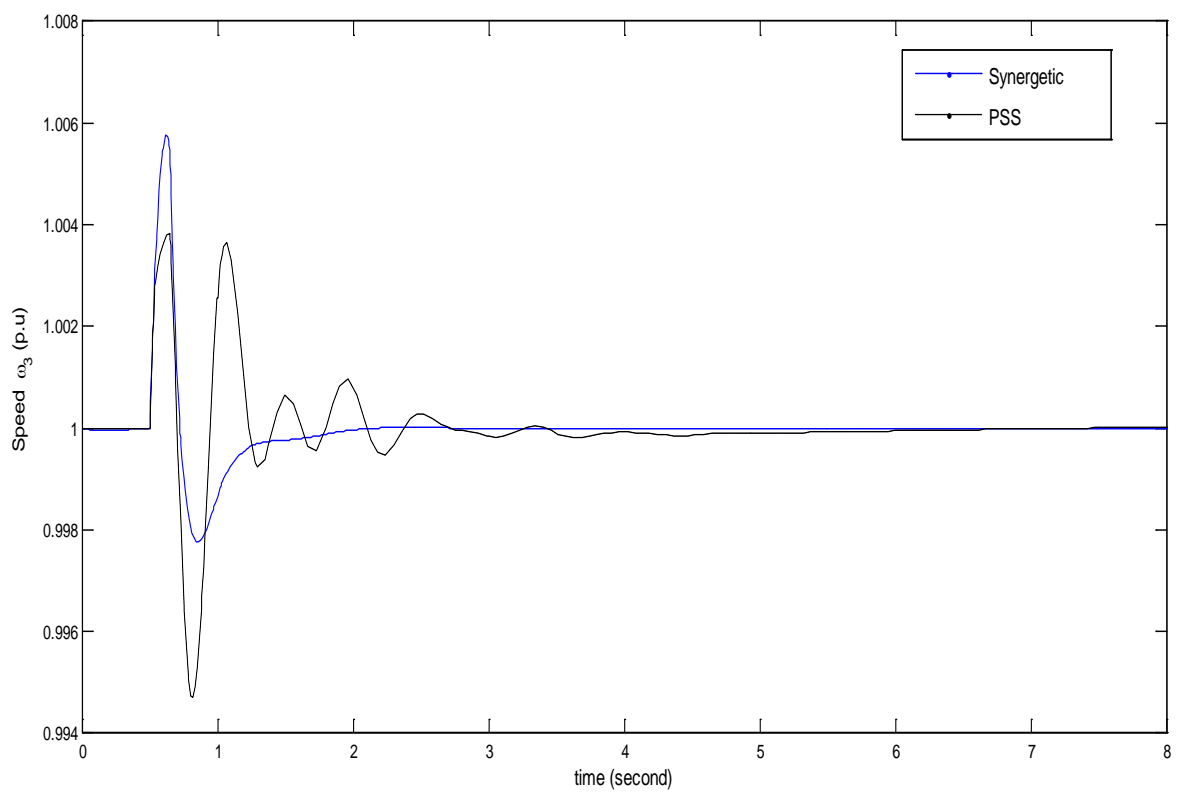

Figure 3.17: Speed of generator 3. (3-Machine Nine Bus System)

Table 3.2: The value of the optimization function

\begin{tabular}{|c|c|c|}
\hline Generator & Synergetic Control & PSS \\
\hline Gen. 1 & 0.009253 & 0.3238 \\
\hline Gen. 2 & 0.004278 & 0.3231 \\
\hline Gen. 3 & 0.01111 & 0.3269 \\
\hline
\end{tabular}

Two Area System (TAS)

The third case study is shown in Figure 3.18. It consists of four generators: two generators in each area. The two areas are connected via a $220 \mathrm{Km}$ tie line and each of the four generators is modeled with transient model and equipped with simple exciter. Two cases are being considered under this study. The first case is when there are two lines between buses 13 and 101 as depicted in Figure 3.18. For this case, a three phase fault is applied on line $3-101$ at time $t=0.1$ second and cleared at $t=0.2$ second but in this case, the line is not reclosed afterward. Synergetic damping controllers are installed at generators 2 and 3. The proposed damping controller is compared with PSS. The 
gains of the PSS had previously been tuned by using Genetic Algorithms (GA) [16]. The transfer functions of the PSS of the generators are:

$$
\begin{aligned}
& G_{2}(s)=47.16 \frac{20 s}{1+20 s} \frac{(1+0.7109 s)^{2}}{(1+0.155 s)^{2}} \\
& G_{3}(s)=300 \frac{20 s}{1+20 s} \frac{(1+0.15 s)^{2}}{(1+0.08431 s)^{2}}
\end{aligned}
$$

With the synergetic controllers' gains chosen to be:

Gen.2: $\alpha_{2}=19.21 ; \Gamma_{12}=18.5 ; \Gamma_{22}=65.045 ; K_{2}=0.775, k_{c 2}=3.172$

Gen.3: $\alpha_{3}=20.1 ; \Gamma_{13}=112.076 ; \Gamma_{23}=0.5 ; K_{3}=0.943 ; k_{c 3}=1.75$

Figures 3.19 to 3.23 show the comparison between the proposed controller and the PSS. The rotor angle differences between generators 3 and 4 with respect to generator 1 are depicted in Figures 3.19 and 3.20 respectively. The speed of generators 2 and 4 are shown in Figures 3.21 and 3.23.

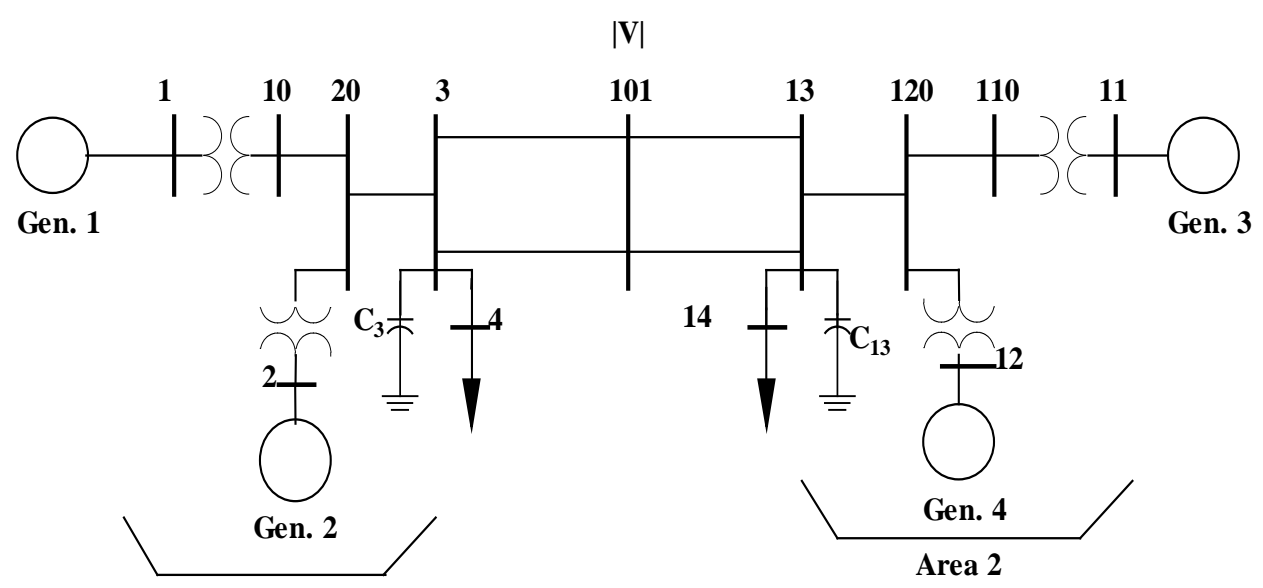

Area 1

Figure 3.18: Single line diagram of two area system 


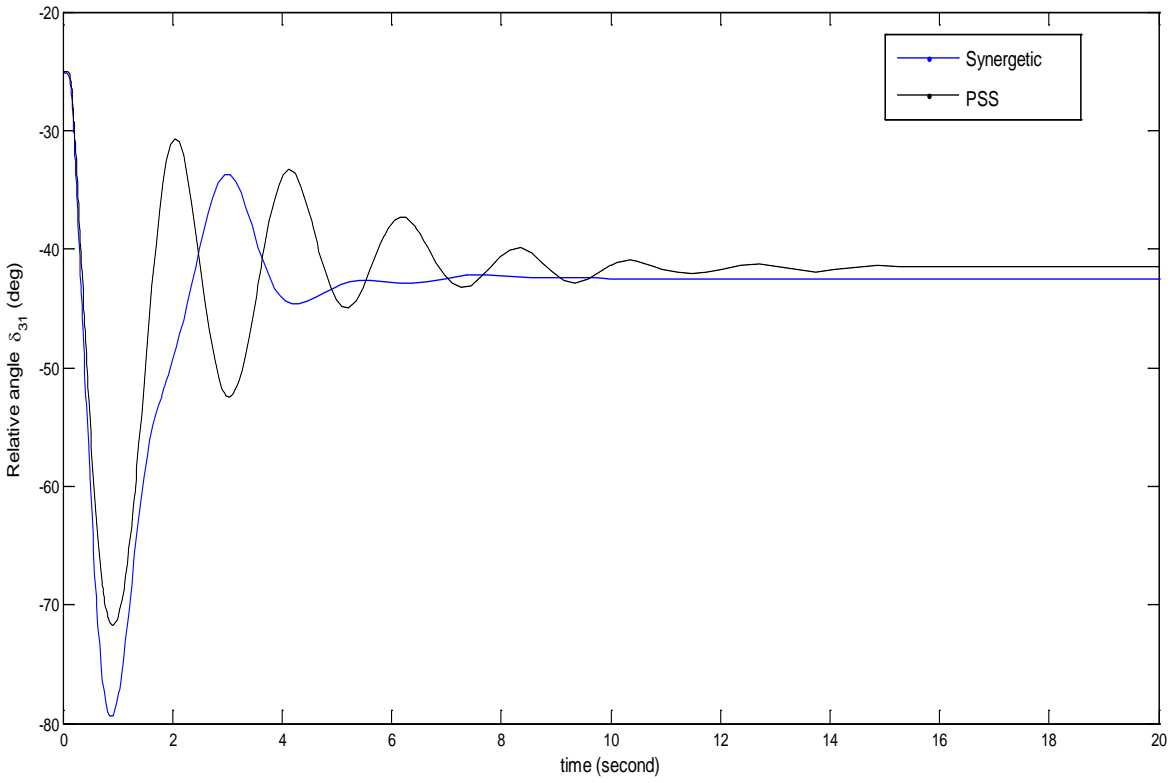

Figure 3.19: Relative rotor angle (degree) 3 - 1for case I (TAS)

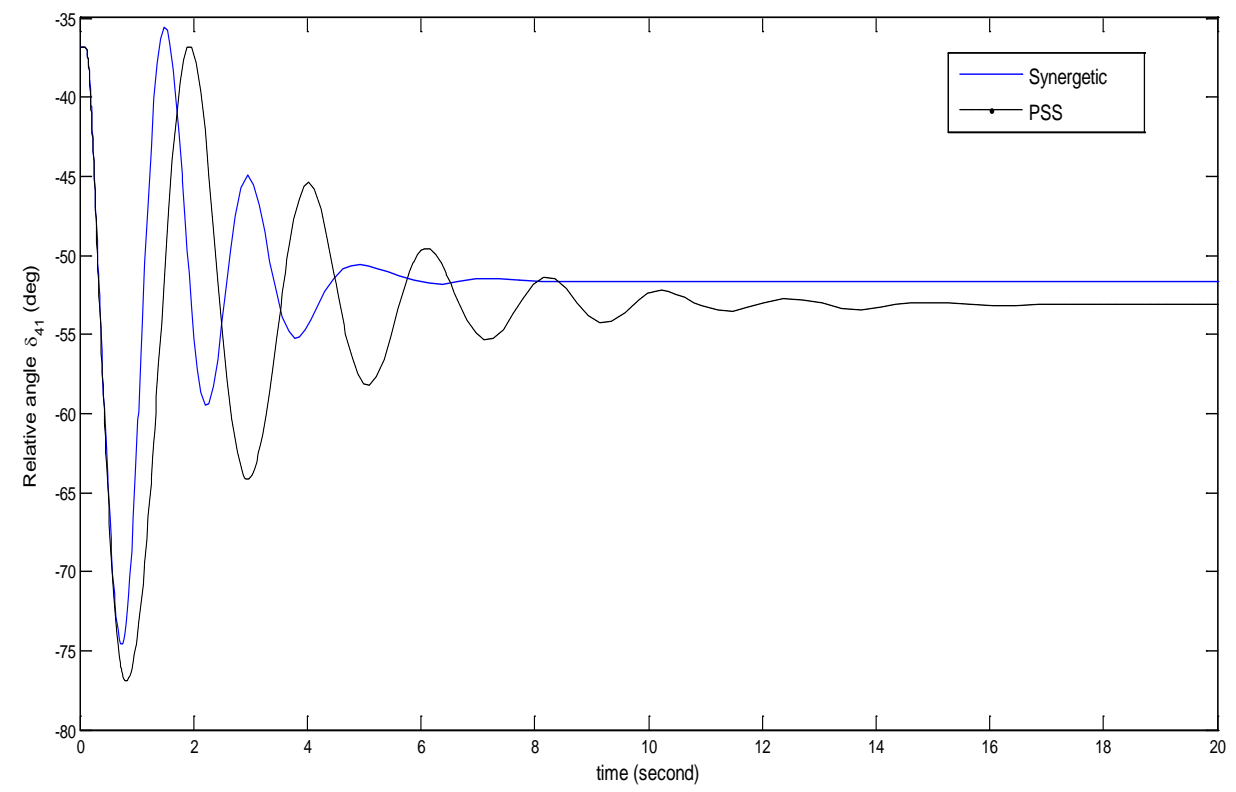

Figure 3.20: Relative rotor angle (degree) 4 - 1 for case I (TAS) 


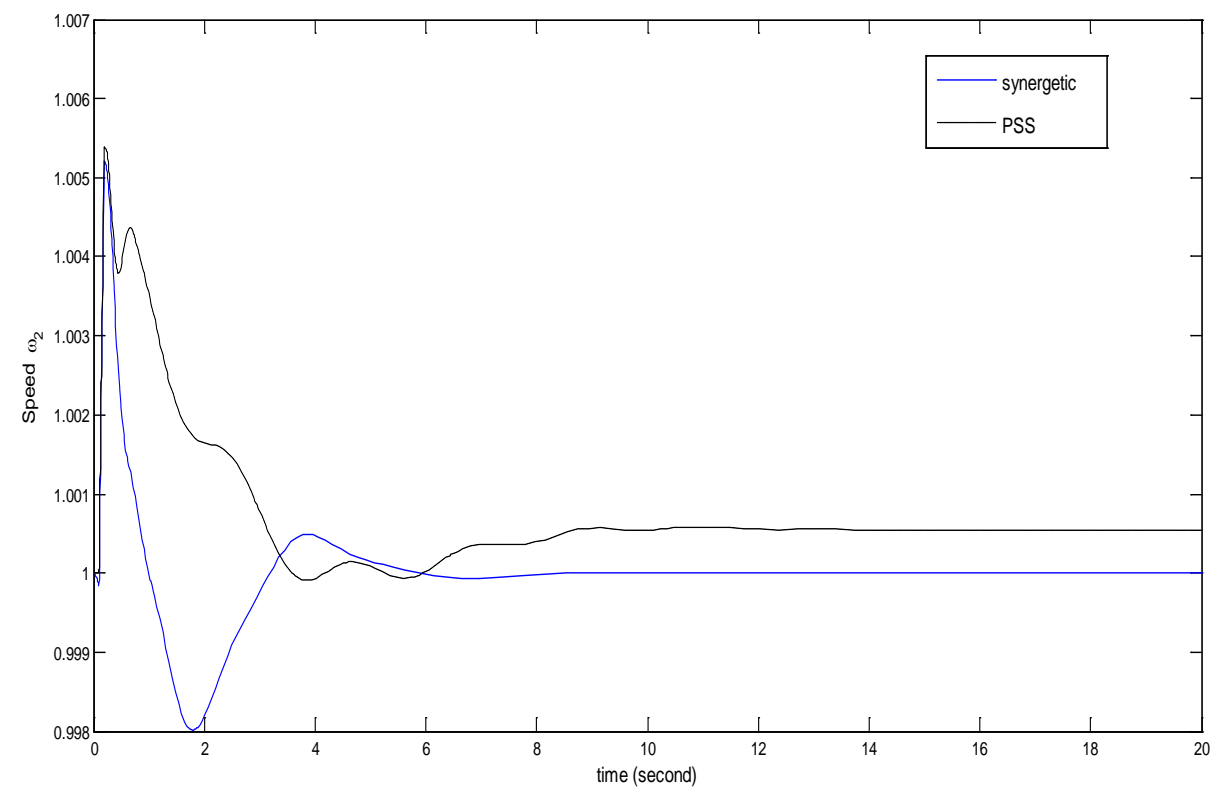

Figure 3.21: Speed of generator 2 for case I

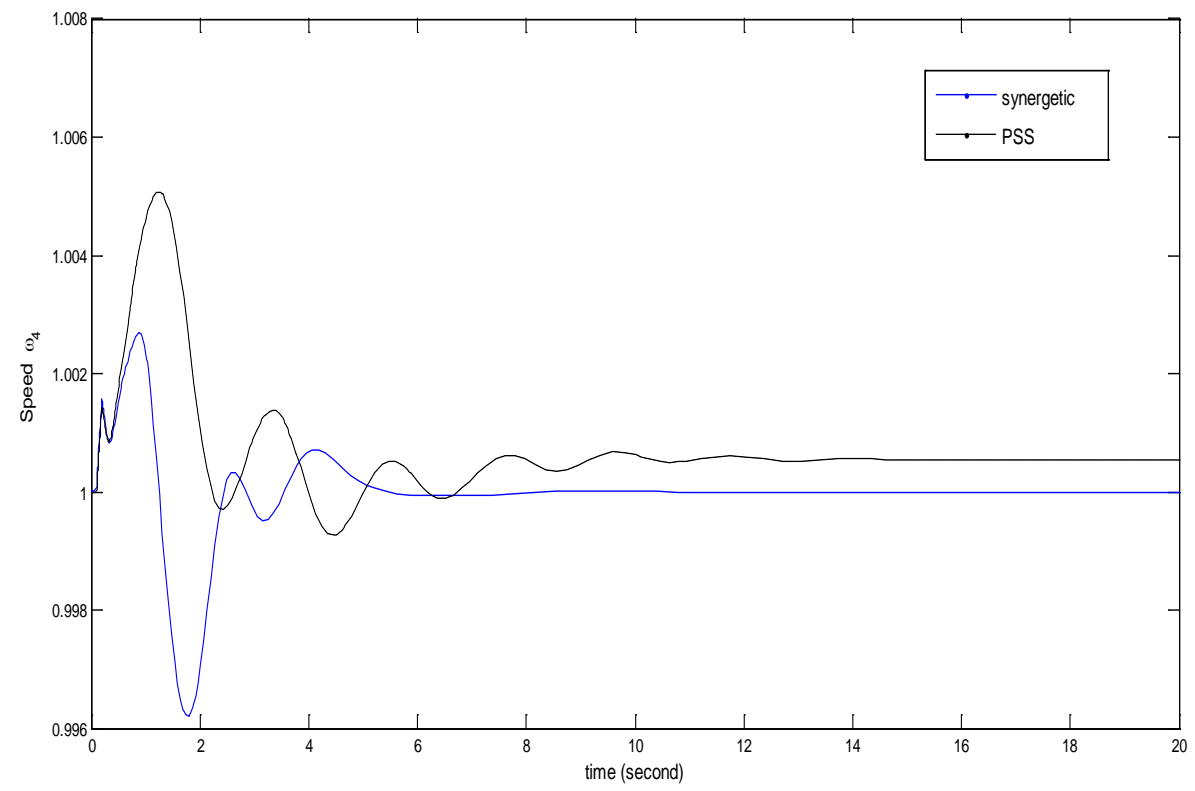

Figure 3.22: Speed of generator 3 for case I (TAS)

The deviation in power in one of the tie lines connecting the two areas is shown in Figure 3.23 and the interconnection terms for generators 2 and 3 are depicted in Figure 3.24. 


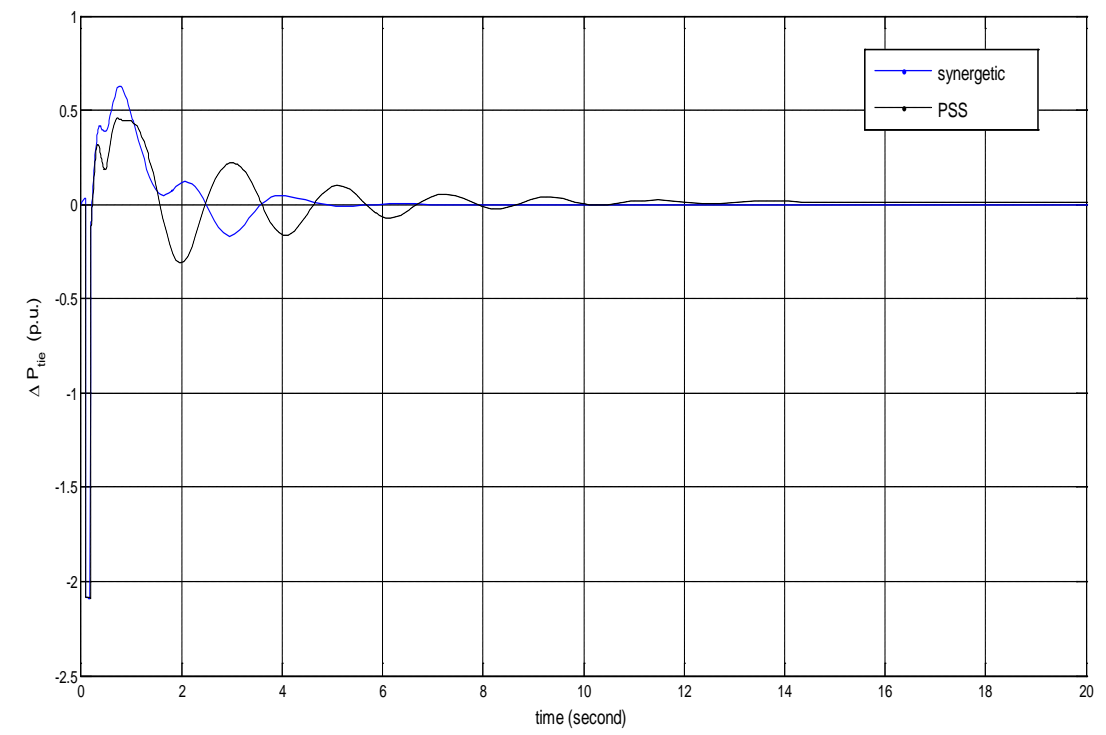

Figure 3.23: Deviation in tie line power (TAS)
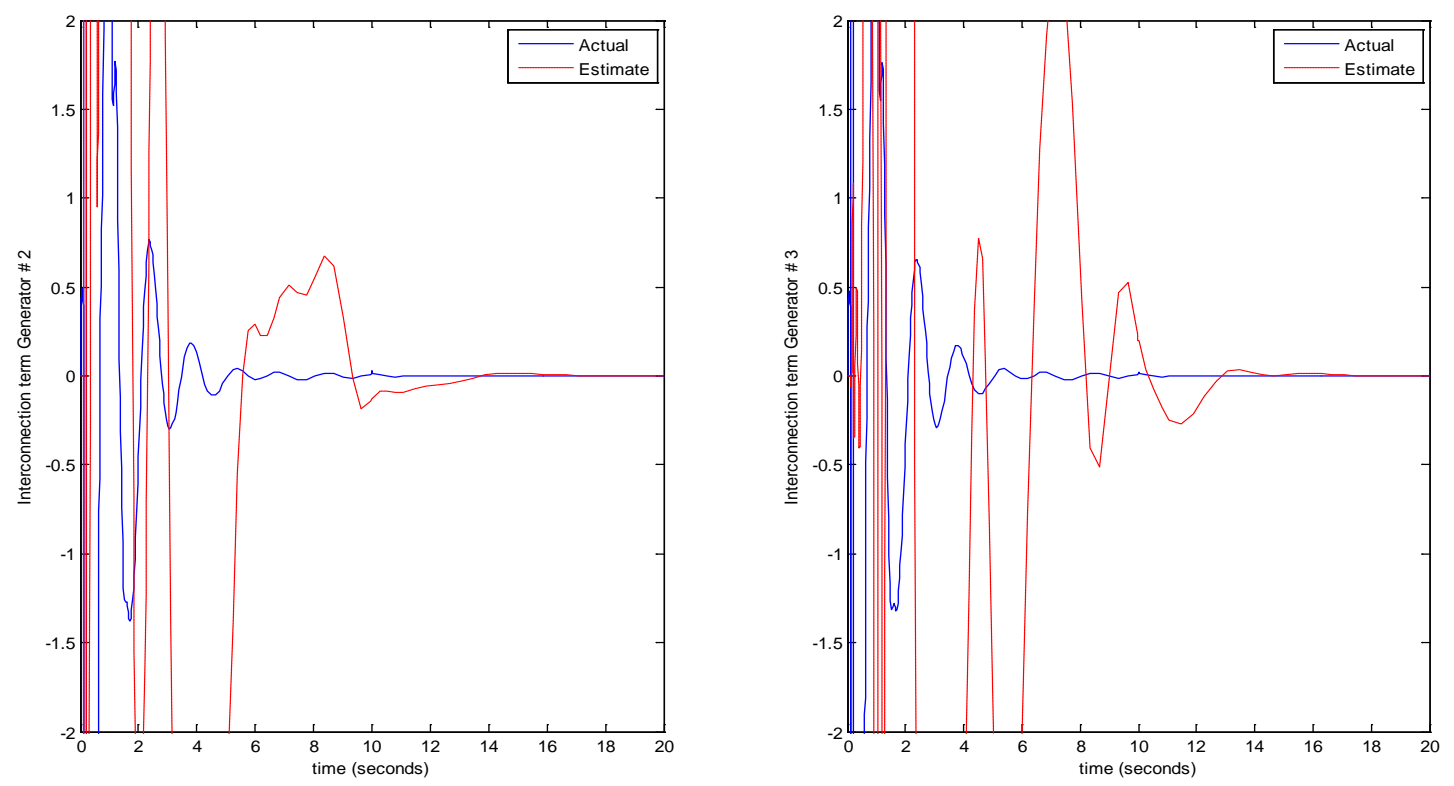

Figure 3.24: The interconnection terms of generators 2 and 3

In the second case, only one transmission line is between buses 13 and 101 . The same fault scenario as in case one is considered and the results are shown in Figures 3.25 - 3.27. The relative rotor angle of generators 3 - 1 and $4-1$ are depicted in Figures 3.25 and 3.26 respectively. It can be seen 
from these Figures that the system is unstable when PSS is employed as the supplementary controllers. Controller signals of generator 2 and 3 are shown in Figure 3.27.

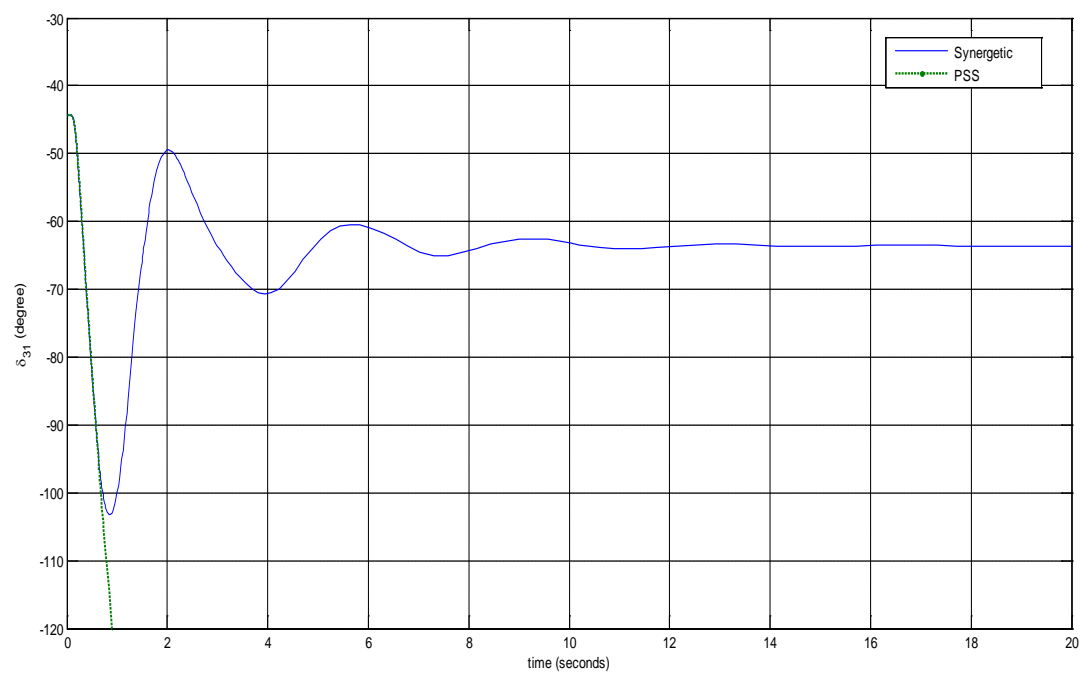

Figure 3.25: Relative rotor angle (degree) 3 - 1 for case II (TAS)

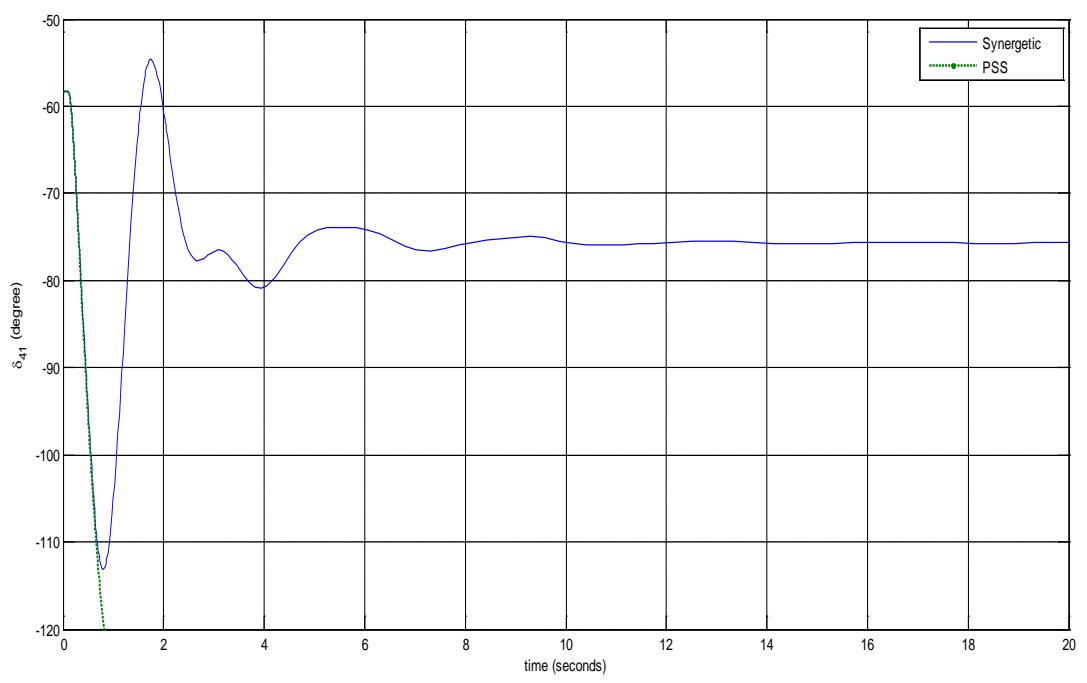

Figure 3.26: Relative rotor angle (degree) 4 - 1 for case II (TAS) 

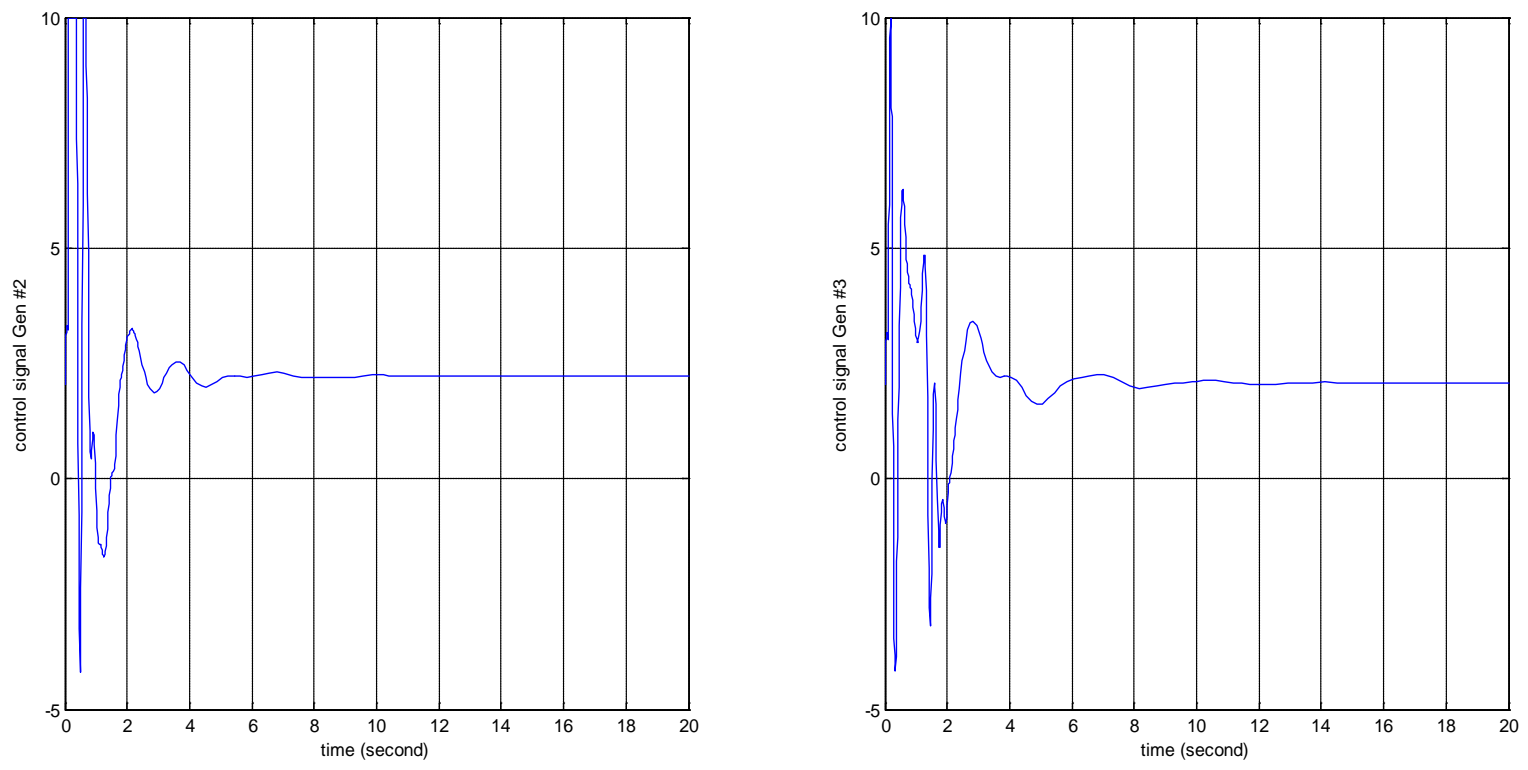

Figure 3.27: Synergetic excitation control signals for Generators 2 and 3

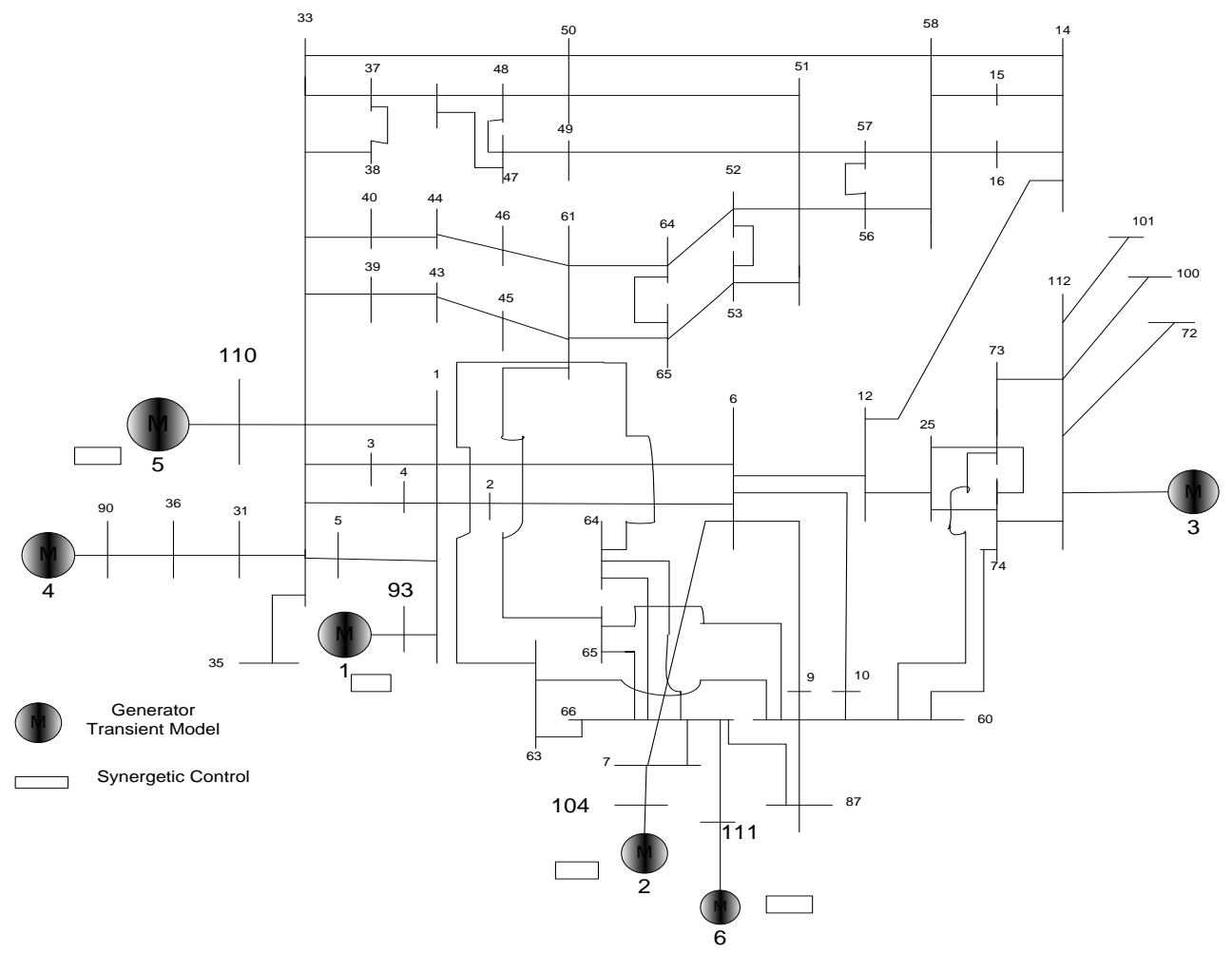

Figure 3.28: Fifty Machine System Single Line Diagram 


\section{Fifty Machine System}

In this case study, the proposed controller design is implemented on the standard mid-sized benchmark system. The system consists of 50 generators, 44 are classical, and 6 are transient models [52]. The single line diagram for this system is depicted in Figure 3.28. Complete data pertaining to generators and exciter data are given in appendix E. Four nonlinear controllers are implemented on generators at buses 93,104, 110 and 111. The parameters of these controllers are tuned with PSO algorithm.

A three phase fault is applied on transmission line $6-9$ at $0.1 \mathrm{sec}$, the fault is cleared and the line is reclosed at $0.25 \mathrm{sec}$. Comparison is done between synergetic control and power system stabilizer. The gains of the PSS are obtained from survey on tuning PSS gains using PSO technique [13]. The gains of the synergetic controllers and that of the PSS are given in Tables 3.2 and 3.3 below.

Table 3.3: Parameters for synergetic Controllers

\begin{tabular}{c|c|c|c|c|c|c}
\hline Gen. \# & Bus \# & $\alpha$ & $\Gamma_{1}$ & $\Gamma_{2}$ & $K$ & $k_{c}$ \\
\hline 1 & 93 & 65 & 23.25 & 31 & 0.895 & 3.5 \\
\hline 2 & 104 & 53.5 & 61.3 & 28.5 & 0.705 & 1.75 \\
\hline 5 & 110 & 65 & 23.25 & 31 & 0.895 & 3.5 \\
\hline 6 & 111 & 53.5 & 61.3 & 28.5 & 0.705 & 1.75 \\
\hline
\end{tabular}

Table 3.4: Parameters for PSS tuned by PSO

\begin{tabular}{|l|l|l|l|l|l|}
\hline Gen. \# & Bus \# & $K_{w}$ & $T_{w}$ & $T_{1}$ & $T_{2}$ \\
\hline 1 & 93 & 93.4223 & 3.4140 & 0.59 & 0.39 \\
\hline 2 & 104 & 28.3032 & 1.6609 & 1.01 & 0.35 \\
\hline 5 & 110 & 81.3726 & 8.5178 & 0.38 & 0.28 \\
\hline 6 & 111 & 7.1390 & 0.5353 & 0.99 & 0.40 \\
\hline
\end{tabular}

In Figures 3.29 and 3.30 synergetic controls damps the rotor oscillations effectively when compared with PSS tuned by PSO, and simple exciter with no damping controller. Controller signals for generators 2 and 6 and their corresponding terminal voltages are depicted Figure 3.31 and 3.32 respectively. 

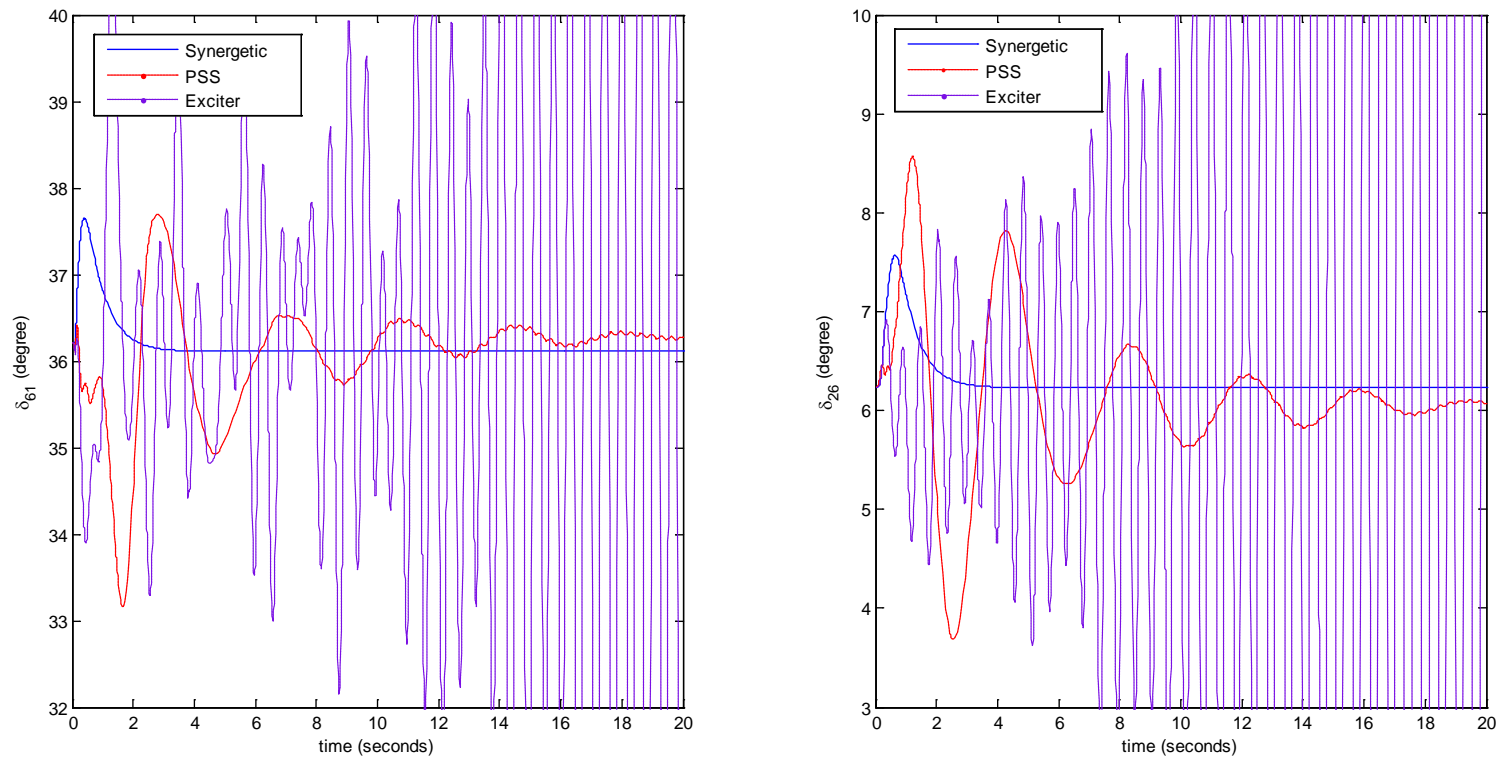

Figure 3.29: Relative rotor angles (degree) 6-1, 2 - 6 (Fifty Machine System)

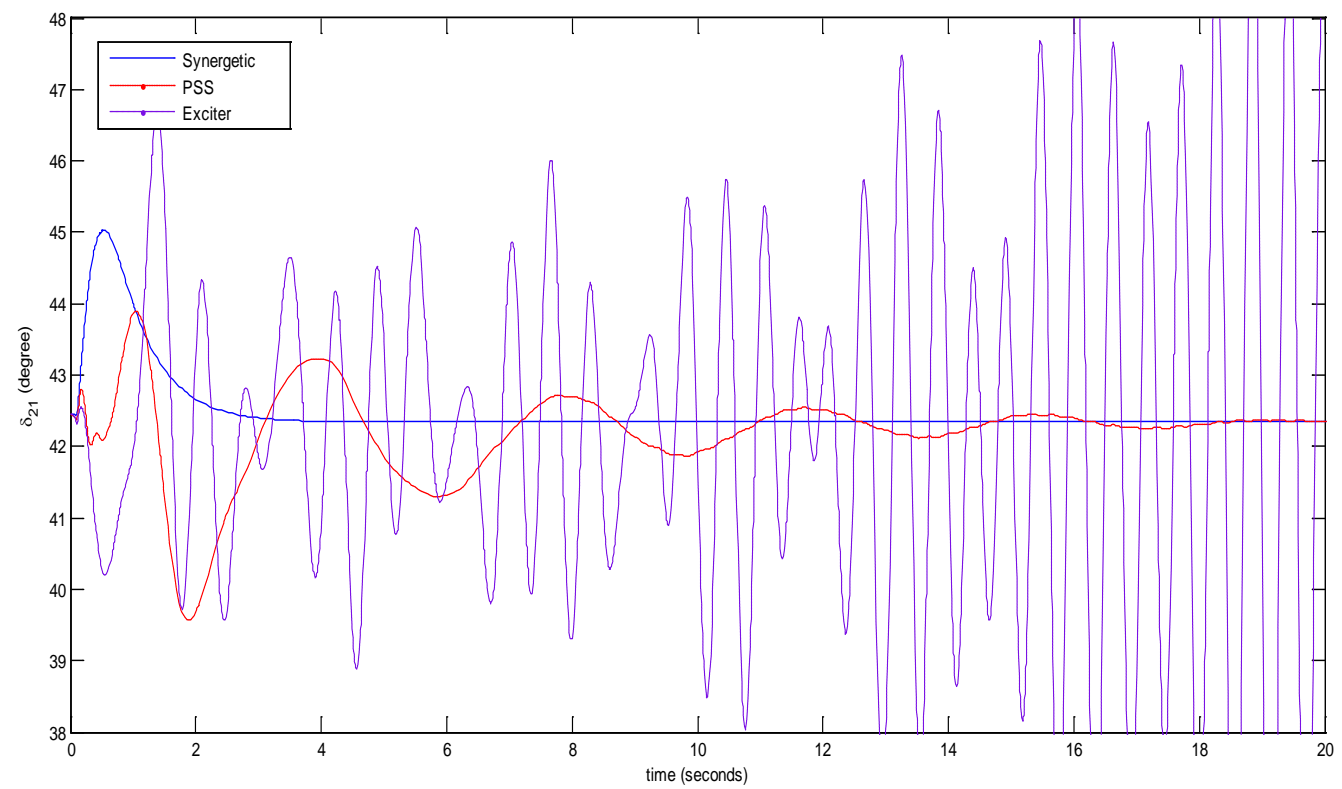

Figure 3.30: Relative rotor angle (degree) 2 - 1 (Fifty Machine System) 

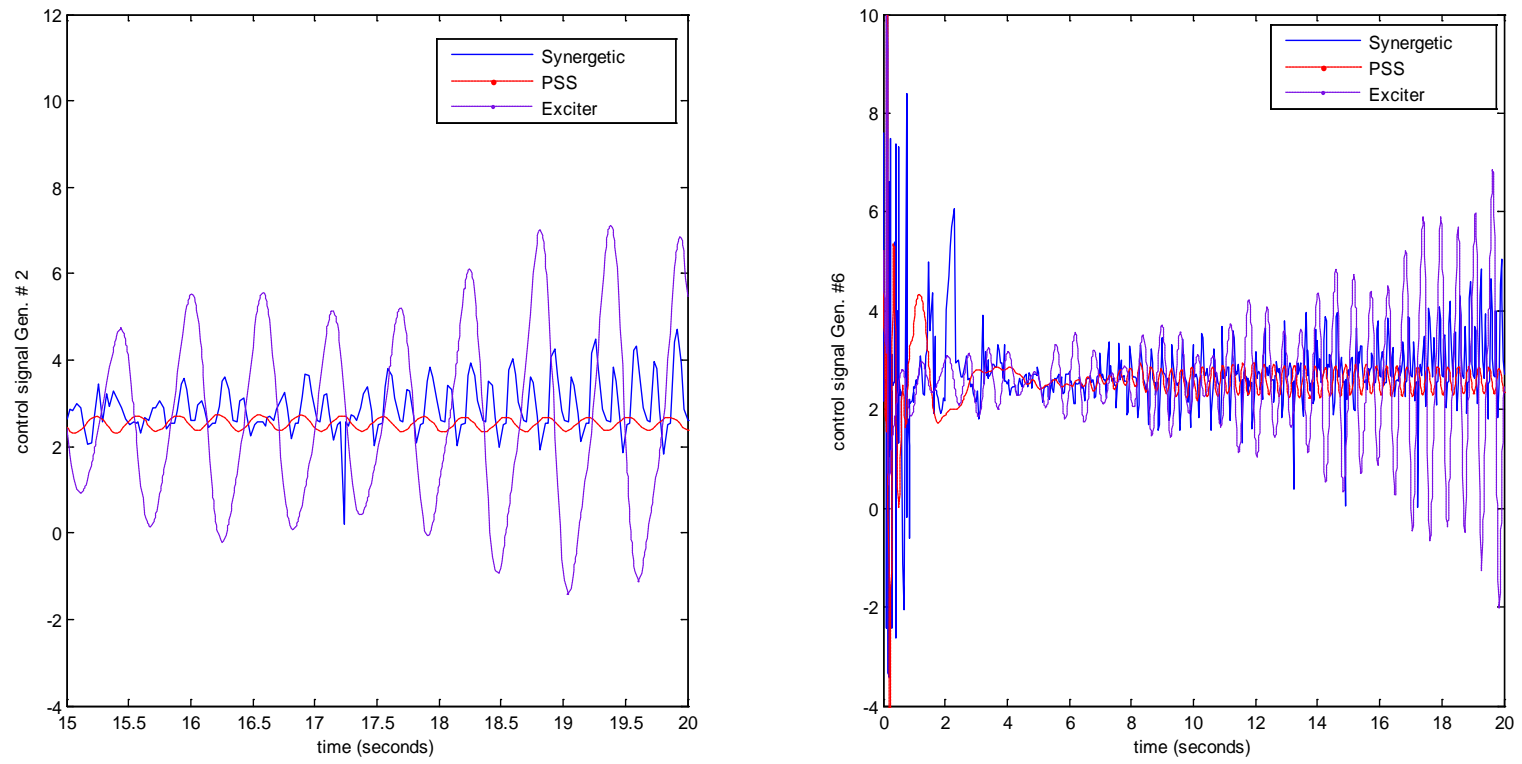

Figure 3.31: Excitation control signals for Generators 2and 6. (Fifty Machine System)
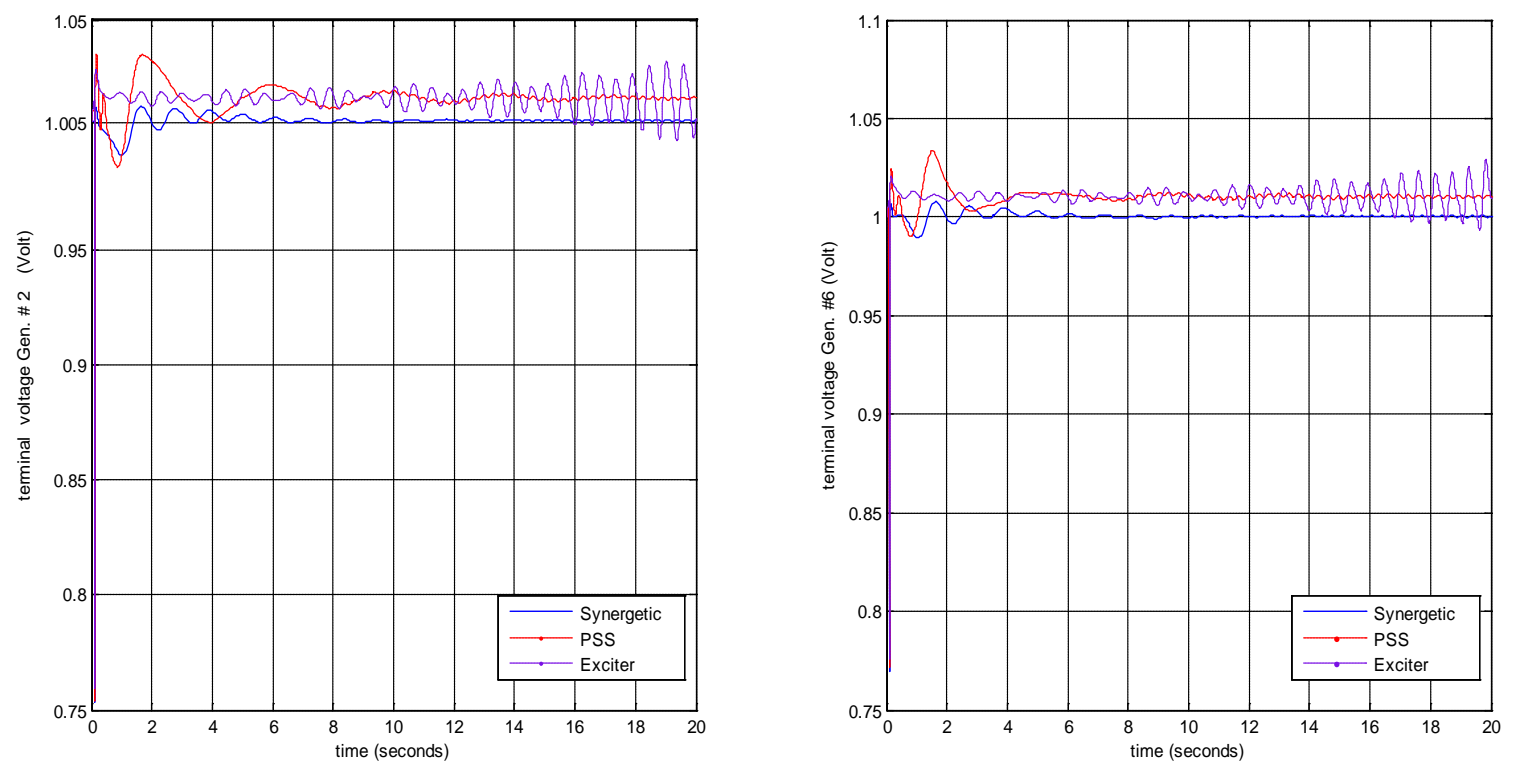

Figure 3.32: Terminal voltages of Generators 2and 6 (Fifty Machine System) 
In all the four case studies presented in this chapter, decentralized synergetic controllers are designed explicitly for the excitation model. The coupling terms between the machines are modeled locally and treated as external disturbance to each of the machines for the second, third and fourth studies. The proposed damping controller is implemented successfully and the results obtained when compared with that of PSS show that the proposed controller is more effective. 


\section{Chapter 4}

\section{Coordination of Synergetic Excitation Controller and SVC Damping Controller}

In the previous chapter, the concept of synergetic control is discussed and the technique is employed to design decentralized controller for power system. The effectiveness of the technique is also discussed. In this chapter, decentralized synergetic controller is coordinated with SVC supplementary controller to enhance the transient stability of the power system.

\subsection{Static Var Compensator}

FACTS technology concepts advance the use of power electronic device to improve the stability of the exiting power system [13]. The devices can be utilized in the prevention of voltage collapse, and enhancements of transient stability and damping of system oscillations. They can enable increase in the transfer of power with little or minimum changes in the system generations or transmissions. Different type of FACTS system is available today. The FACTS device or system that is utilized in this work is Static Var Compensator (SVC). The term "static" is used to indicate the absence of moving or rotating component in the device. The most common topology is the fixed capacitor - thyristor controlled reactor type as depicted in Figure 4.1. The SVC consists of a capacitor bank, a thyristor controlled reactor, and a transformer that connects the SVC to the high voltage power system. The device is capable of controlling the voltage of the bus to which it is connected. The current flowing through the inductor is adjusted by modifying the firing angle $\alpha$ of the thyristor. As a result of this, the reactance of the SVC can be change from inductive to capacitive. The range of the firing angle is $\left[\frac{\pi}{2}, \pi\right], \frac{\pi}{2}$ for inductive reactance and $\pi$ for capacitive reactance. 


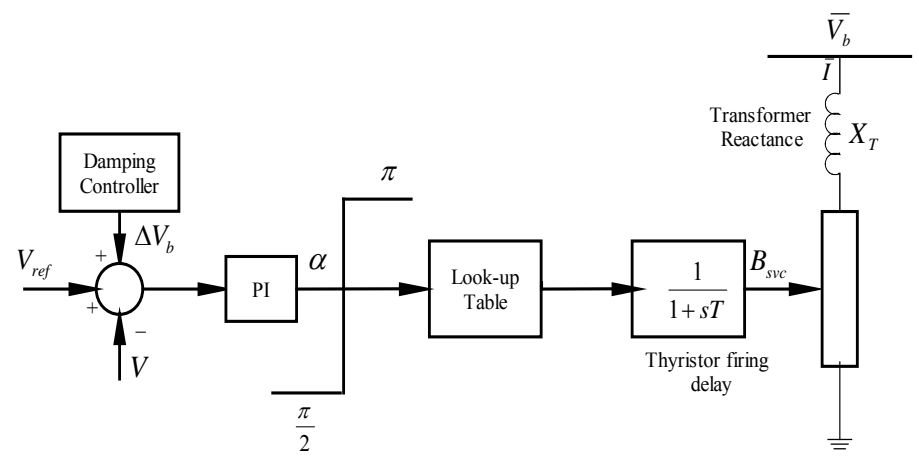

Figure 4.1: SVC Equivalent Circuit Model

The bus where SVC is connected can be considered to be a generator bus where injected active power is equated to zero and the required reactive power is computed through load flow algorithm. The desired steady state values at the SVC bus are obtained as follow:

$$
\begin{aligned}
& \bar{I}=\left(\frac{j Q_{b}}{\bar{V}_{b}}\right)^{*} \\
& \bar{V}=\bar{V}_{b}+j X_{T} \bar{I} \\
& B=j \frac{\bar{I}}{\bar{V}} \\
& B_{s v c}(\alpha)=\frac{2 \alpha-\sin (2 \alpha)-\pi\left(2-\frac{X_{L}}{X_{C}}\right.}{\pi X_{L}}
\end{aligned}
$$

where $Q_{b}$ and $\bar{V}_{b}$ are the injected reactive power and bus voltage as determined by the load flow algorithm, $\bar{I}$ is the current into the bus, $\bar{V}$ is the voltage across the SVC, $X_{T}$ is the reactance of the transformer, and $B_{s v c}$ is the equivalent admittance of the SVC. The actual admittance of SVC is obtained from the pre-calculated look up table using the values of $X_{L}$ and $X_{C}$ and the range of the firing angle. The dynamic of the thyristor firing delay is approximated by a first order block with the delay time constant assumed to be about $3 \mathrm{msec}$. PI controller is employed to set the firing angle within its range. This controller is termed the internal controller of the SVC. 
In order to improve the performance of the SVC, a damping supplementary controller is designed. The input to this controller can be local signals such as bus voltage, active power flow or the line current. It can also be remote signals such as generator speed or difference between speeds of two generators belonging to different power system area. In this work, the structure of the SVC supplementary controller adopted is lead-lag compensator (see Figure 4.2)

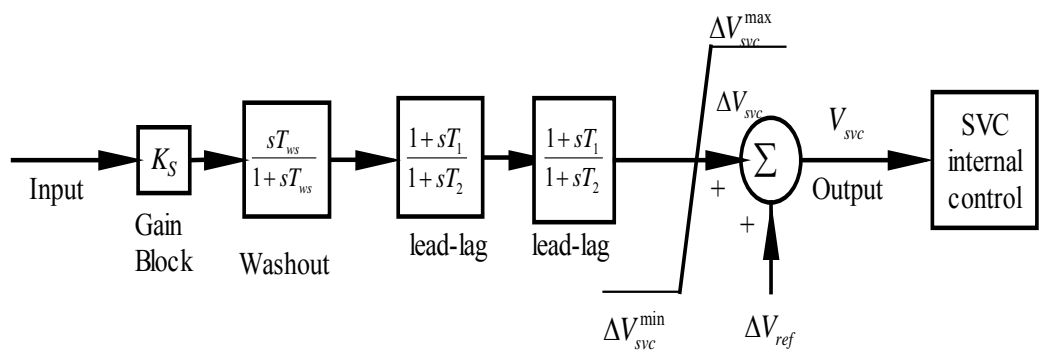

Figure 4.2: Structure of the SVC - based supplementary controller

The controller consists of a gain block, a washout block and two-stage phase compensator. The wash out block provides the high -pass filtering function that permits signals associated with oscillations in input signal to pass unaltered and the phase compensation acts to provide the necessary phase - lead characteristics to compensate for any phase lag between the input and the output signals. . The output signal $U_{\text {out }}$ is fed into the SVC internal controller. The proportional and the integral gains of the controller are fixed at 20 and 0.3 respectively. The output of this controller modifies the firing angle of the thyristor and this translates into modification in the susceptance of the SVC.

The SVC is coordinated with synergetic excitation controller by simultaneously tuning the gain of the SVC supplementary controller and the parameters of the synergetic controller using PSO technique. The approach is implemented on single and multiple machine power system.

\subsection{Single Machine Infinite Bus}

A single machine infinite bus system with AC transmission link is analyzed in this case [3]. The system is given by nonlinear differential and algebraic equations including generator, SVC with supplementary controller, load and an infinite bus. The schematic diagram of the system is shown 
in Figure 4.3 and parameters for generator and line reactance are given in Table 4.1. The main function of Static Var Compensator is to increase transmittable power by regulating the bus voltage and suppressing power oscillations.

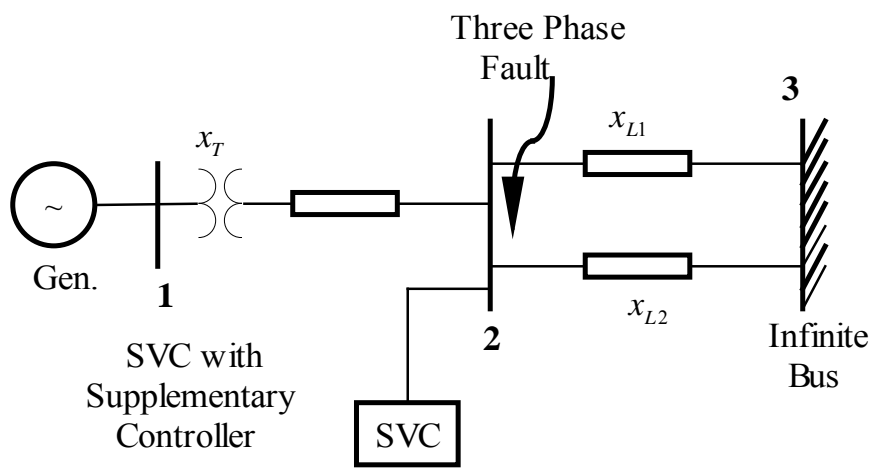

Figure 4.3: Single Machine Infinite Bus System with SVC

The generator is modeled by a third- order single axis model as:

Mechanical equations:

$$
\begin{aligned}
& \Delta \dot{\delta}(t)=\omega_{o} \Delta \omega(t) \\
& \Delta \dot{\omega}(t)=-\frac{D}{2 H} \Delta \omega(t)-\frac{1}{2 H}\left(P_{e}(t)-P_{m}(t)\right)
\end{aligned}
$$

Generator electrical dynamics:

$$
\dot{E}_{q}^{\prime}(t)=\frac{1}{T_{d o}^{\prime}}\left(E_{f}(t)-\left(x_{d}-x_{d}^{\prime}\right) I_{d}-E_{q}^{\prime}(t)\right)
$$

Electrical equations:

$$
E_{f}(t)=k_{c} u_{f}(t)
$$




$$
\begin{aligned}
& P_{e}(t)=\frac{E_{q}^{\prime}(t) V_{s}}{x_{d s}^{\prime}} \sin \delta(t) \\
& Q_{e}(t)=\frac{E_{q}^{\prime}(t) V_{s} \cos \delta(t)-V_{s}^{2}}{x_{d s}^{\prime}} \\
& I_{q}(t)=\frac{V_{s}}{x_{d s}^{\prime}} \sin \delta(t) \\
& I_{d}(t)=\frac{E_{q}^{\prime}(t)-V_{s} \cos \delta(t)}{x_{d s}^{\prime}}
\end{aligned}
$$

By substituting (4.11) in (4.9) and differentiating the result we have:

$$
\begin{aligned}
\dot{P}_{e}(t) & =\dot{E}_{q}^{\prime} I_{q}+E_{q}^{\prime} \dot{I}_{q} \\
\dot{P}_{e}= & \frac{1}{T_{d o}^{\prime}}\left(E_{f}-\left(x_{d}-x_{d}^{\prime}\right) I_{d}-E_{q}^{\prime}\right) I_{q}+ \\
& +E_{q}^{\prime}\left(\frac{V_{s}(\cos \delta) \dot{\delta}}{x_{d s}^{\prime}}-\frac{V_{s} \sin \delta}{x_{d s}^{\prime}} \frac{x_{1} x_{2} \dot{B}_{s v c}}{x_{d s}^{\prime}}\right) \\
\Delta \dot{P}_{e}= & -\frac{1}{T_{d o}^{\prime}} \Delta P_{e}+\frac{1}{T_{d o}^{\prime}}\left(k_{c} u_{f}-\left(x_{d}-x_{d}^{\prime}\right) I_{d}-P_{m}\right) I_{q} \\
& +Q_{e} \omega_{o} \Delta \omega+d
\end{aligned}
$$

Where

$$
d=\frac{V_{s}^{2} \omega_{o} \Delta \omega}{x_{d s}^{\prime}}-\frac{X_{1} X_{2}}{x_{d s}^{\prime}} P_{e} \dot{B}_{s v c}
$$

By defining $x_{1}=\Delta \delta=\delta-\delta_{o}, \quad x_{2}=\Delta \omega=\omega-1, \quad x_{3}=\Delta P_{e}=P_{e}-P_{m}, \quad$ and representing the interaction between the generator and the rest of the system as $d=c_{1} x_{3}+c_{2} x_{3}^{2}$, with $c_{1}$ and $c_{2}$ unknown constant but to be determined by using adaptation law, (4.5), (4.6) and (4.15) can be expressed as: 
Table 4.1: Parameters of Generator and Transmission Lines

\begin{tabular}{l|l}
\hline Leakage reactance (p.u.) & $x_{l}=0.15$ \\
\hline Resistance (p.u.) & $r_{a}=0.003$ \\
\hline d-axis reactance (p.u.) & $x_{d}=1.81$ \\
\hline q-axis reactance (p.u.) & $x_{q}=1.494$ \\
\hline d-axis transient reactance (p.u.) & $x_{d}^{\prime}=0.3$ \\
\hline d-axis open circuit time constant (sec) & $T_{d o}^{\prime}=8.0$ \\
\hline Inertia constant (sec) & $H=4.0$ \\
\hline Reactance AC line (p.u.) & $x_{L 1}=0.93$ \\
\hline Reactance AC line (p.u.) & $x_{L 2}=0.5$ \\
\hline Transformer + line reactance & $x_{T}=0.2$ \\
\hline$x_{1}, x_{2}, x_{d s}^{\prime}$ & $x_{1}=x_{d}^{\prime}+x_{T}$ \\
& $x_{2}=x_{L 1} / / x_{L 2}$ \\
& $x_{d s}^{\prime}=x_{1}+x_{2}-x_{1} x_{2} B_{S V C}$ \\
\hline
\end{tabular}

$$
\begin{aligned}
& \dot{x}_{1}=\omega_{o} x_{2} \\
& \dot{x}_{2}=-\frac{D}{2 H} x_{2}-\frac{1}{2 H} x_{3} \\
& \dot{x}_{3}=-\frac{1}{T_{d o}^{\prime}} x_{3}+\frac{1}{T_{d o}^{\prime}} v_{f}+d
\end{aligned}
$$

where $v_{f}$ is a new variable defined as:

$$
v_{f}=k_{c} I_{q} u_{f}-\left(x_{d}-x_{d}^{\prime}\right) I_{d} I_{q}-P_{m}+T_{d o}^{\prime} Q_{e} \omega_{o} X_{2}
$$

We can see from (4.20) that the mapping from $u_{f}$ to $v_{f}$ is invertible, except when $I_{q}(t)=0$, which is not in the working region of the generator.

\subsubsection{Controller Design}

\section{Synergetic Excitation Controller}

The procedure of synergetic control technique has been discussed in detail in the previous chapter. By defining the macro-variable to be: 


$$
\varphi=\left(\frac{d^{2}}{d t^{2}}+\alpha \frac{d}{d t}+\alpha^{2}\right) x_{1}
$$

where $\alpha>0$ is a design parameter, and following the procedure explained in chapter 2 , the control input signal $u_{f}$ can be obtained as:

$$
\begin{aligned}
u_{f}= & \frac{1}{k_{c} I_{q}}\left(x_{3}+T_{d o}^{\prime}\left\{2 H\left(\alpha-\frac{D}{2 H}+\frac{1}{K}\right) \dot{x}_{2}+2 H \alpha\left(\alpha+\frac{1}{K}\right) x_{2}+\right.\right. \\
& \left.\left.\frac{2 H \alpha^{2}}{\omega_{o} K} x_{1}-\hat{d}\right\}+P_{m}+\left(x_{d}-x_{d}^{\prime}\right) I_{d} I_{q}-T_{d o}^{\prime} Q_{e} \omega_{o} x_{2}\right)
\end{aligned}
$$

where:

$$
\begin{aligned}
& \hat{d}=\hat{c}_{1} x_{3}+\hat{c}_{2} x_{3}^{2} \\
& \dot{\hat{c}}_{1}=\Gamma_{1} \varphi x_{3} \\
& \dot{\hat{c}}_{2}=\Gamma_{2} \varphi \dot{x}_{3}
\end{aligned}
$$

The parameters of the synergetic controller are $\alpha, K, \Gamma_{1}, \Gamma_{2}$ and $k_{c}$.

\section{SVC Supplementary Damping Controller}

The structure of the damping controller adopted in this work has been discussed in the previous section. The SVC supplementary controller gains are $K_{S}, T_{w s}, T_{1}$ and $T_{2}$.

Both of these controllers are coordinated together by simultaneously tuning the parameters of the synergetic controller and the gains of the SVC supplementary controller using particle swarm optimization. The objective function $J$ to be optimized by PSO is given as:

$$
\min _{\text {Find } \phi} J=\int_{0}^{t} k_{1}(\Delta \delta)^{2}+k_{2}(\Delta \omega)^{2}+k_{3}\left(\Delta V_{T}\right)^{2}+k_{4}\left(\Delta V_{s v c}\right)^{2}
$$


where $\Delta V_{T}$ and $\Delta V_{s v c}$ are the deviations in the terminal voltage of the generator and the SVC bus voltage respectively. $\phi=\left[\alpha, K, \Gamma_{1}, \Gamma_{2}, k_{c}, K_{S}, T_{s w}, T_{1}, T_{2}\right], k_{1}, k_{2}, k_{3}$ and $k_{4}$ are weighing factors.

The following scenarios are presented to illustrate the effectiveness of the approach proposed here.

- Effect of short circuit fault.

- Effective of level of power generation.

Effect of short circuit fault: This scenario show the effectiveness of the coordination of the two controllers when a three phase short circuit fault occurs on one of the AC transmission lines. The proposed approach is compared with:

- Only SVC supplementary damping controller is present [51]

- Only Synergetic controller is present at the exciter side [47]

- Only power system stabilizer is present at the exciter side.

In each of the four cases, the generator is also equipped with a static exciter [3]. The parameters of the exciter can be found in the Appendix. The three phase fault occurs on transmission line $2-3$ (Figure 4.3) at $0.5 \mathrm{sec}$. The fault is cleared by removing the line after $40 \mathrm{msec}$, and the line is reconnected after $60 \mathrm{msec}$. Table 4.2 depicts the tuned controller gains. Figures 4.4 and 4.5 depict the relative angle and the speed of the generator.

Table 4.2: Damping Controller Gains for SMIB System

\begin{tabular}{c|c|c|c}
\hline $\begin{array}{c}\text { SVC Supplementary } \\
\text { Damping Controller }\end{array}$ & PSS Controller & Synergetic Controller & $\begin{array}{c}\text { Synergetic Controller } \\
\text { with SVC }\end{array}$ \\
\hline$K_{s}=80.1749$ & $K_{S}=13.775$ & $\alpha=10.3131$ & $K_{S}=3.3564$ \\
\hline$T_{w s}=6.4087$ & $T_{w s}=1.41$ & $K=0.228$ & $T_{w s}=9.9978$ \\
\hline$T_{1}=0.1687$ & $T_{1}=0.154$ & $k_{c}=1$ & $T_{1}=0.7009$ \\
\hline$T_{2}=0.4753$ & $T_{2}=0.033$ & & $T_{2}=7.009$ \\
\hline & & & $\alpha=13.5$ \\
\hline & & & $K=0.75$ \\
\hline & & & $k_{c}=3.5$ \\
\hline & & & $\Gamma_{1}=7.9677$ \\
\hline
\end{tabular}




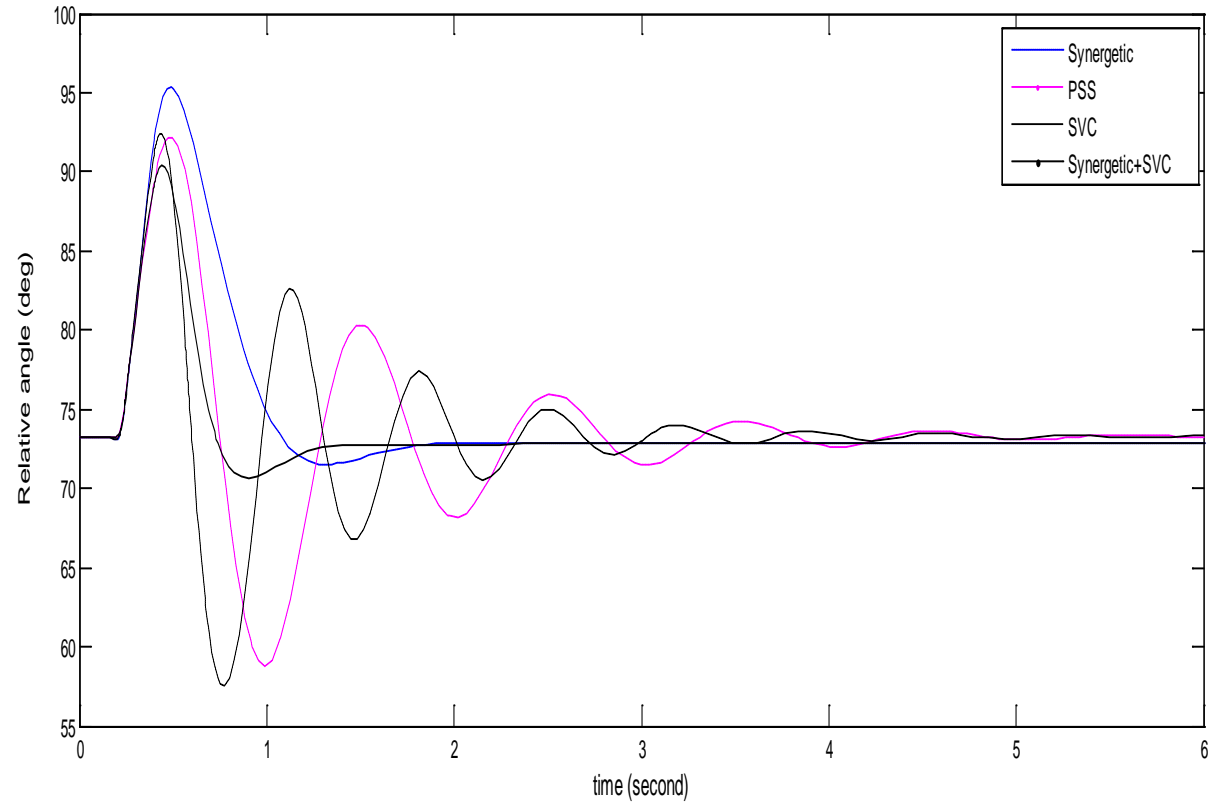

Figure 4.4: Power angle of the generator

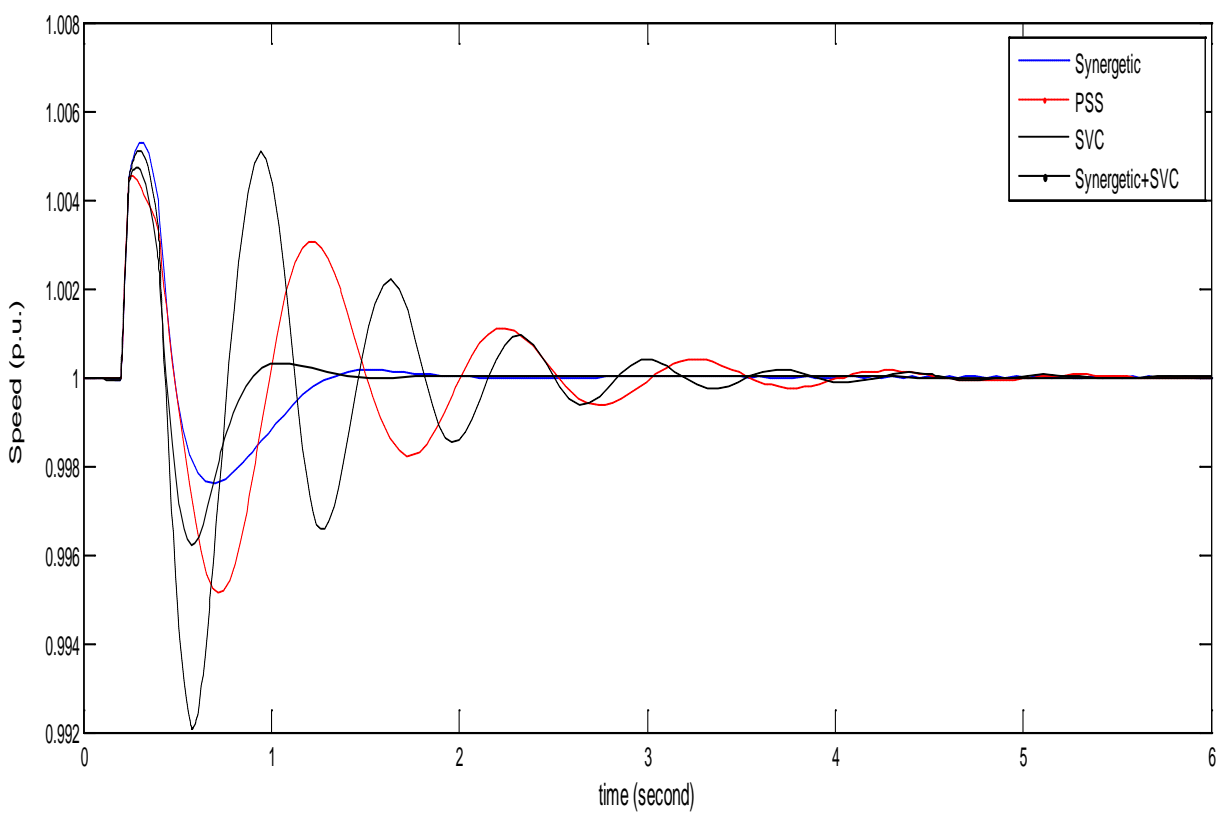

Figure 4.5: Speed of the generator 
Effect of level of power generation: In this scenario effect of low power generation to high power generation is looked into. Load flow solution for each bus is obtained for each set of power generation. Figures 4.6 and 4.7 depict power and speed deviation.

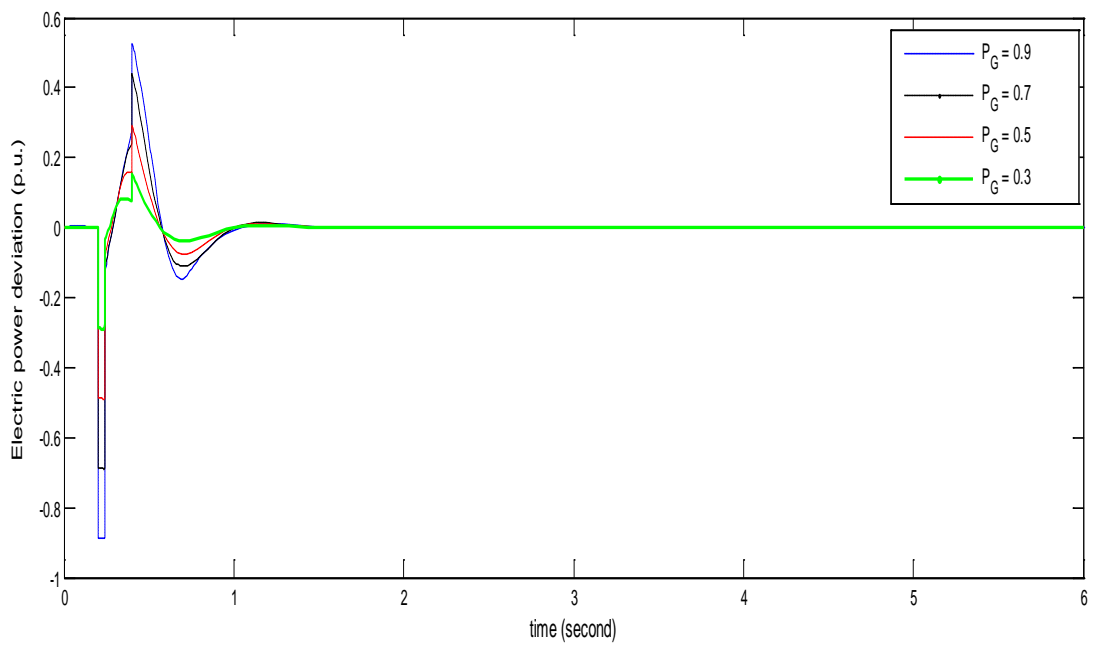

Figure 4.6: Effect of low to high generation power on the system electric power

Figure 4.7 shows the effect of low to high power generation on performance of coordination of synergetic controller and SVC supplementary damping controller. Figure 4.9 shows the impacts on the terminal voltage of the generator.

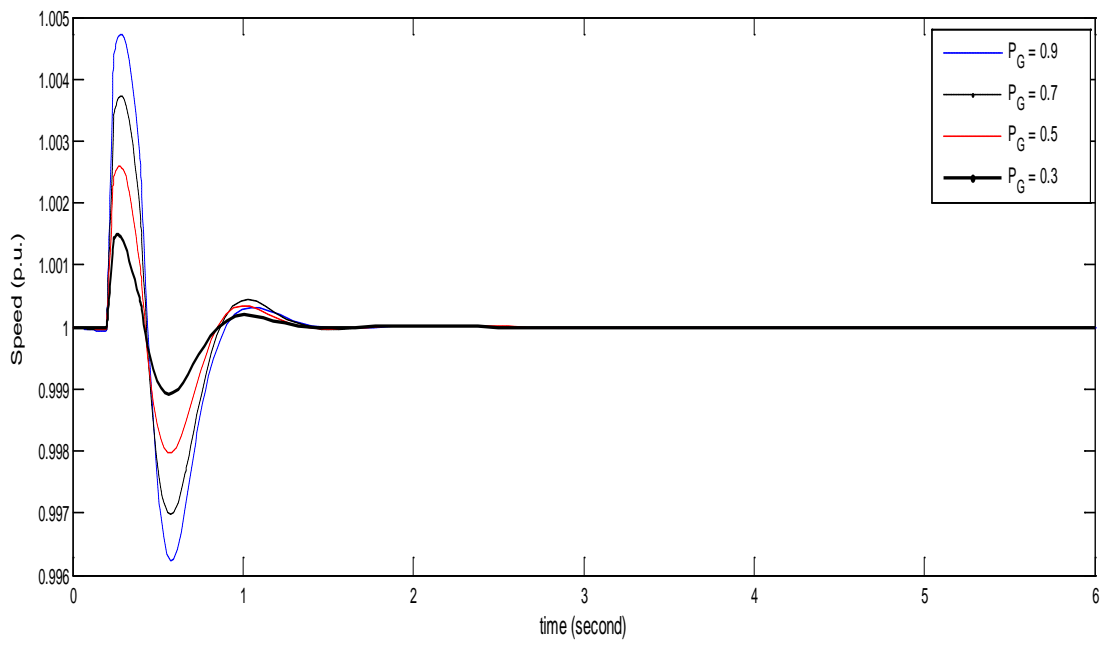

Figure 4.7: Effect of low to high generation power on speed of the generator 


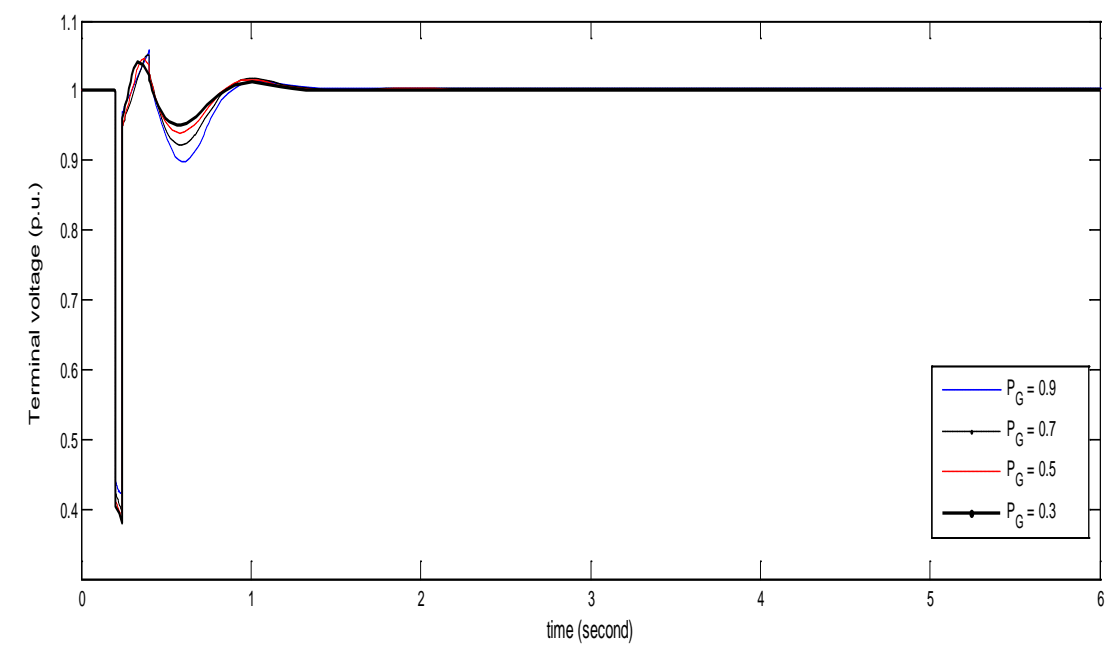

Figure 4.8: Effect of low to high generation power on terminal voltage of the generator

\subsection{Two Area System}

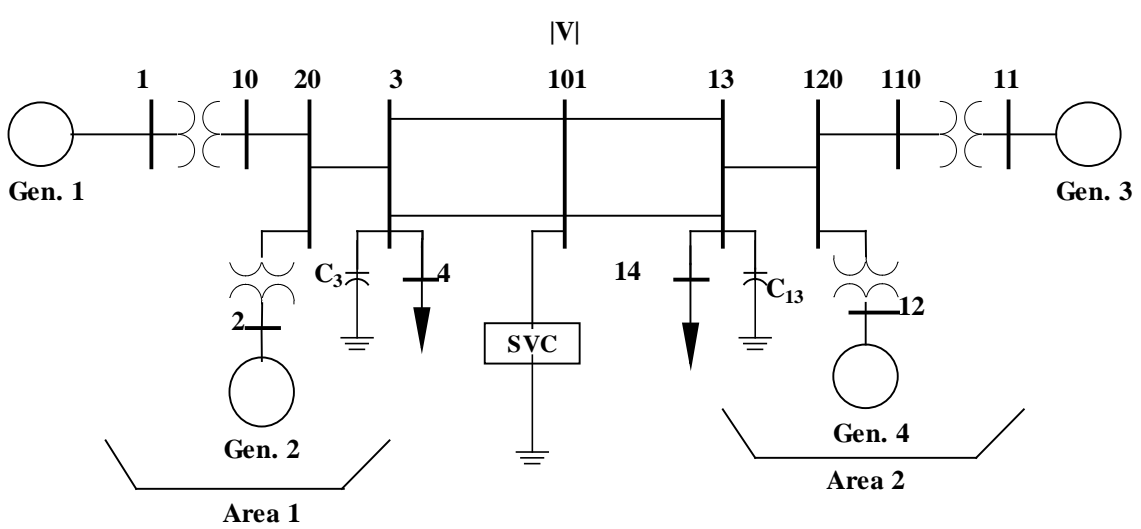

Figure 4.9: Two Area System with SVC device

A two area four machine system is considered for this study. The single line diagram for this system is shown in Figure 4.9. The two areas are identical with two parallel tie lines which transfer 400 MW from area 1 to area 2 in normal operating condition. Generators parameters can be found in [3]. Simple exciter is installed in each of the four generators. 
Synergetic damping controller for multimachine system [47] had been discussed in the previous chapter. The dynamics of the $i^{t h}$ generator is given as:

$$
\begin{aligned}
& \dot{x}_{1 i}=\omega_{o} X_{2 i} \\
& \dot{x}_{2 i}=-\frac{D_{i}}{2 H_{i}} x_{2 i}-\frac{1}{2 H_{i}} x_{3 i} \\
& \dot{x}_{3 i}=-\frac{1}{T_{d o i}^{\prime}} x_{3 i}+\frac{1}{T_{d o i}^{\prime}} v_{f i}+d_{i}
\end{aligned}
$$

where $d_{i}$, the interaction between the $i^{\text {th }}$ generator and the rest of the system, is given as:

$$
\begin{aligned}
d_{i}= & E_{q i}^{\prime} \sum_{j=1}^{n} \dot{E}_{q j}^{\prime} B_{i j} \sin \left(\delta_{i}-\delta_{j}\right)- \\
& E_{q i}^{\prime} \sum_{j=1}^{n} E_{q j}^{\prime} B_{i j} \cos \left(\delta_{i}-\delta_{j}\right)\left(\omega_{j}\right)
\end{aligned}
$$

The synergetic damping controller obtained is given to be:

$$
\begin{aligned}
u_{f i} & =\frac{1}{k_{c i} I_{q i}}\left\{P_{\text {mio }}+\left(x_{d i}-x_{d i}^{\prime}\right) I_{d i} I_{q i}+T_{d o i}^{\prime} Q_{e i} x_{2 i}\right. \\
& +\left(1-T_{d o i}^{\prime} \hat{c}_{1 i}\right) x_{3 i}-T_{d o i}^{\prime} \hat{c}_{2 i} x_{3 i}^{2}+\frac{2 H_{i} T_{d o i}^{\prime} \alpha_{i}^{2}}{\omega_{o} K_{i}} x_{1 i} \\
& +2 H_{i} T_{d o i}^{\prime}\left(\alpha_{i}-\frac{D_{i}}{2 H_{i}}+\frac{1}{K_{i}}\right) \dot{x}_{2 i}+2 H_{i} T_{d o i}^{\prime}\left(\alpha_{i}^{2}+\frac{\alpha_{i}}{K_{i}}\right) x_{2 i}
\end{aligned}
$$

where $\hat{c}_{1 i}$ and $\hat{c}_{2 i}$ are given as:

$$
\begin{aligned}
& \hat{c}_{1 i}=\Gamma_{1 i} \varphi_{i} x_{3 i} \\
& \hat{c}_{2 i}=\Gamma_{2 i} \varphi_{i} x_{3 i}^{2}
\end{aligned}
$$

The definition of each of the variables is given in Table 4.1. In this study, synergetic controllers are 
installed on generators 2 and 3, and a SVC is placed at bus 101. A supplementary controller is designed for the SVC using the structure described in the previous section. The input signal to this controller is the tie line power in one of the lines connecting the two areas together. The parameters of the two synergetic decentralized controllers and the gains of the SVC damping controller is then simultaneously tuned using PSO to obtain their optimum values. The objective function to be optimized is given as:

$$
\begin{aligned}
J= & \int_{0}^{t}\left(\sum_{i=1}^{4} k_{1 i}\left(\Delta \delta_{i 1}\right)^{2}+k_{2 i}\left(\Delta \omega_{i}\right)^{2}+k_{3 i}\left(\Delta V_{T i}\right)^{2}\right. \\
& \left.+k_{4}\left(\Delta V_{s v c}\right)^{2}+\sum_{j=1}^{2} k_{5 j}\left(\Delta P_{t_{i e_{-}} j}\right)^{2}\right)
\end{aligned}
$$

A three phase fault occurs on line $3-101$ at $0.1 \mathrm{sec}$. The fault is cleared by removing the line at 0.190 sec. The line is then reconnected at $0.24 \mathrm{sec}$. Two cases are considered in this study. The first case is when only synergetic controllers are installed at generators 2 and 3 [47]. The second case is the coordination of these controllers with the SVC damping controller. The results obtained for the two cases are given below:

\section{Synergetic controllers without SVC.}

Generator 2:

$\alpha_{2}=27.5519, K_{2}=0.946, k_{c 2}=5.32, \Gamma_{21}=18.0618, \Gamma_{22}=30.7813$

Generator 3:

$\alpha_{3}=28.8901, K_{3}=0.8806, k_{c 3}=5.7895, \Gamma_{31}=36.4021, \Gamma_{32}=0.3819$

\section{Synergetic controllers with SVC}

\section{Generator 2}

$\alpha_{2}=30.2376, K_{2}=1.5801, k_{c 2}=3.5628, \Gamma_{21}=14.309, \Gamma_{22}=4.8233$

\section{Generator 3}

$\alpha_{3}=29.2058, K_{3}=1.2720, k_{c 3}=5.7237, \Gamma_{31}=35.4854, \Gamma_{32}=8.1182$

SVC Supplementary Controller

$K_{s}=3.5949, T_{w s}=10, T_{1}=0.7655, T_{2}=7.655$ 
The results obtained are simulated and compared to each other. Comparison is also made with previous study when PSSs are installed at generators 2 and 3 [16]. The results of the simulation are shown in the Figures 4.10 to 4.15 . Figures 4.10 and 4.11 show the relative angles between generators 3 and 1 and between generator 4 and 1 .

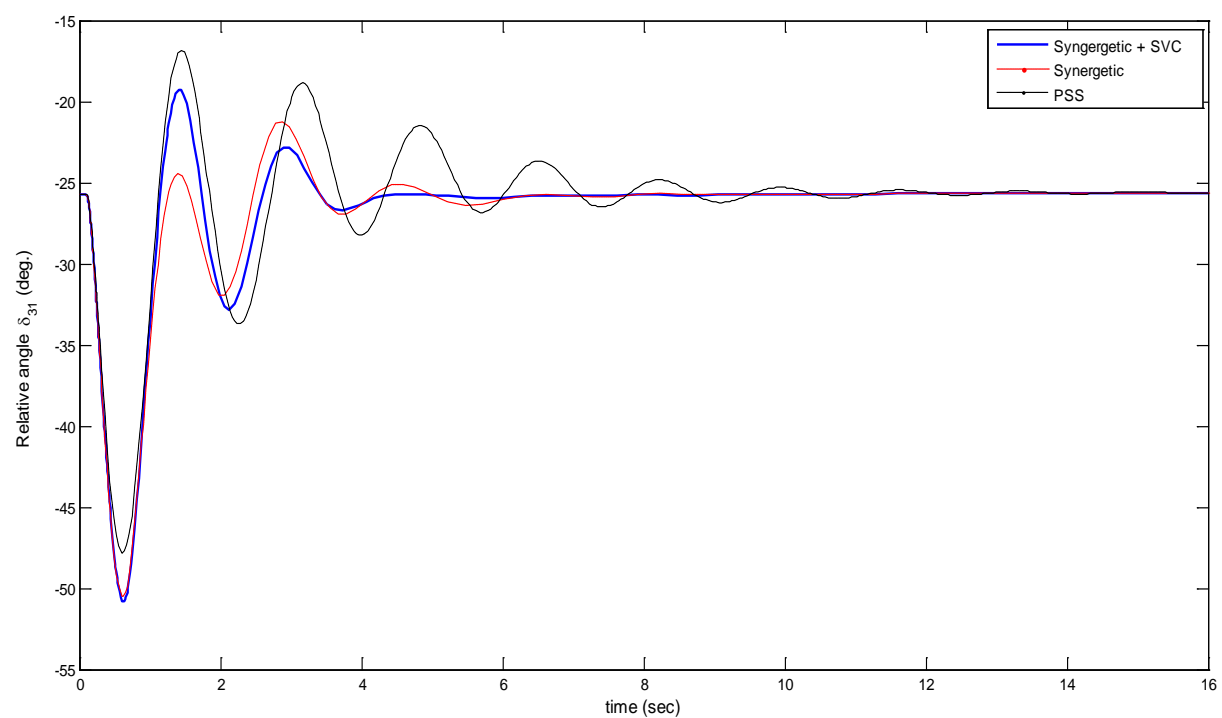

Figure 4.10: Relative power angle (degree) 3 - 1

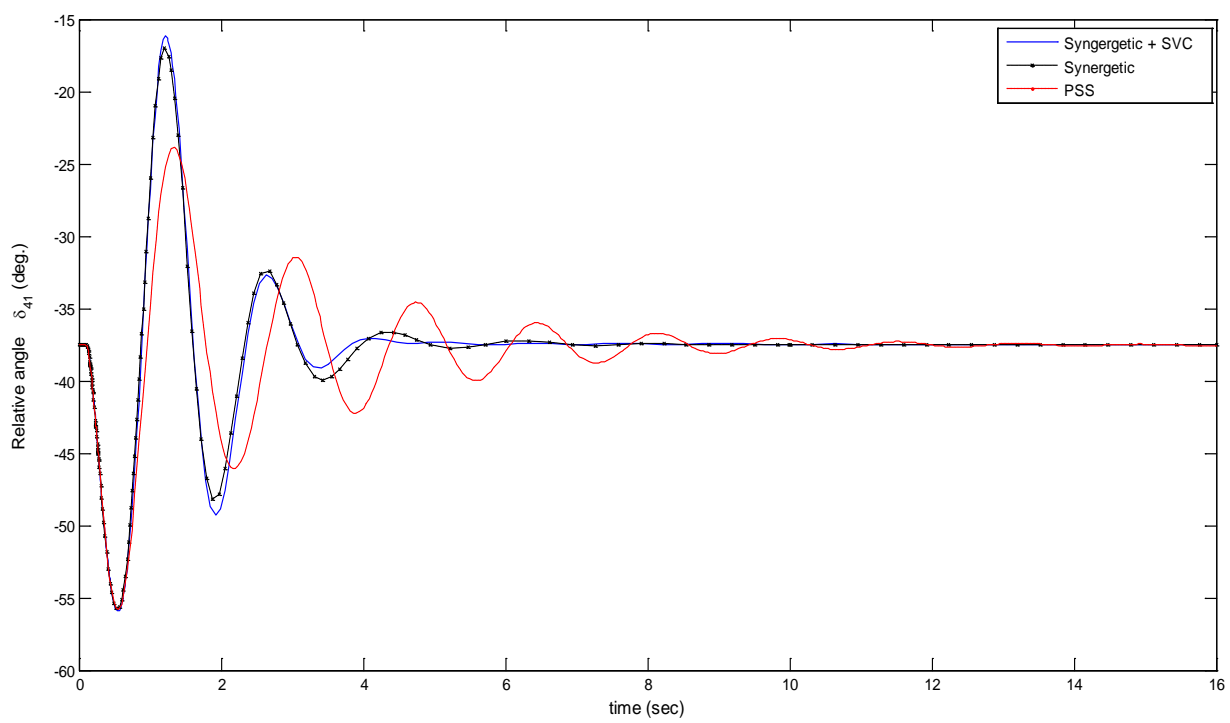

Figure 4.11: Relative power angle (degree) 4 - 1 


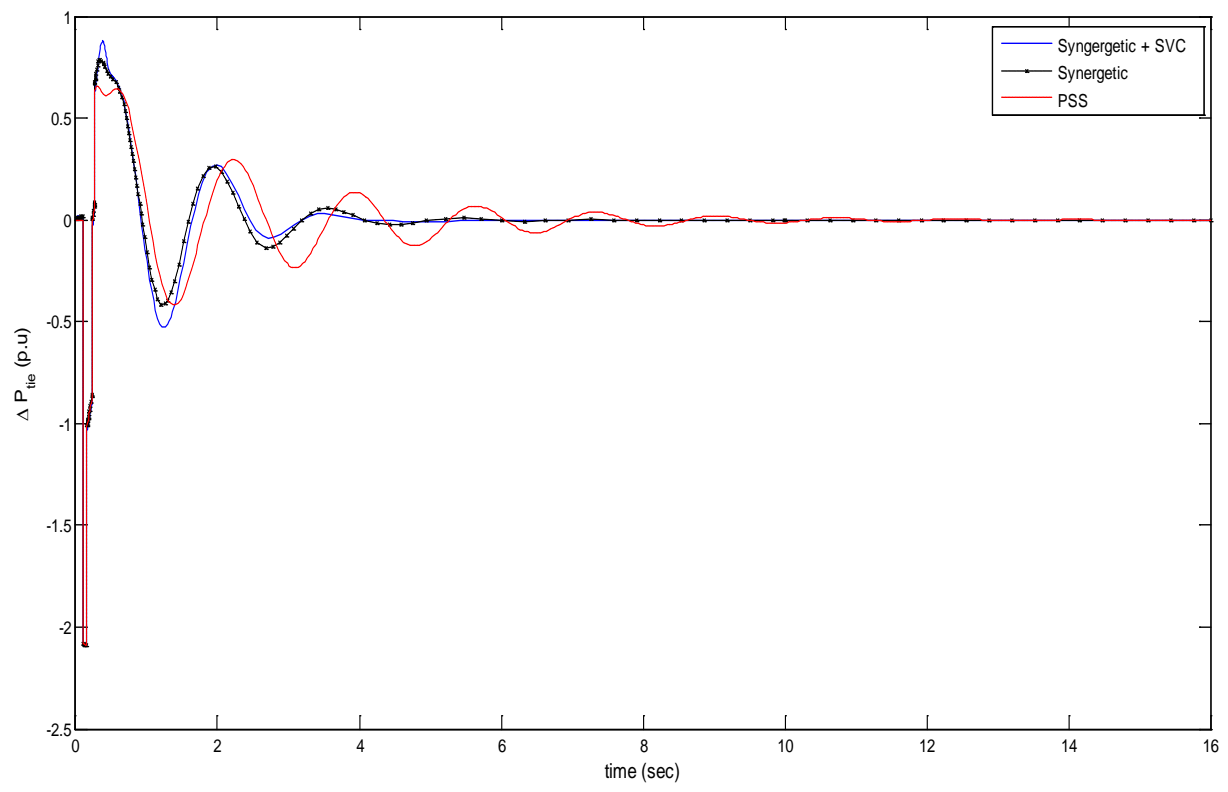

Figure 4.12: Tie -line power deviation on line 3 - 101

The deviation in power transfer through one of the AC transmission lines connecting the two areas together is depicted in Figure 4.12. The speed of generators 2 and 3 are also depicted in Figures 4.13 and 4.14 respectively.

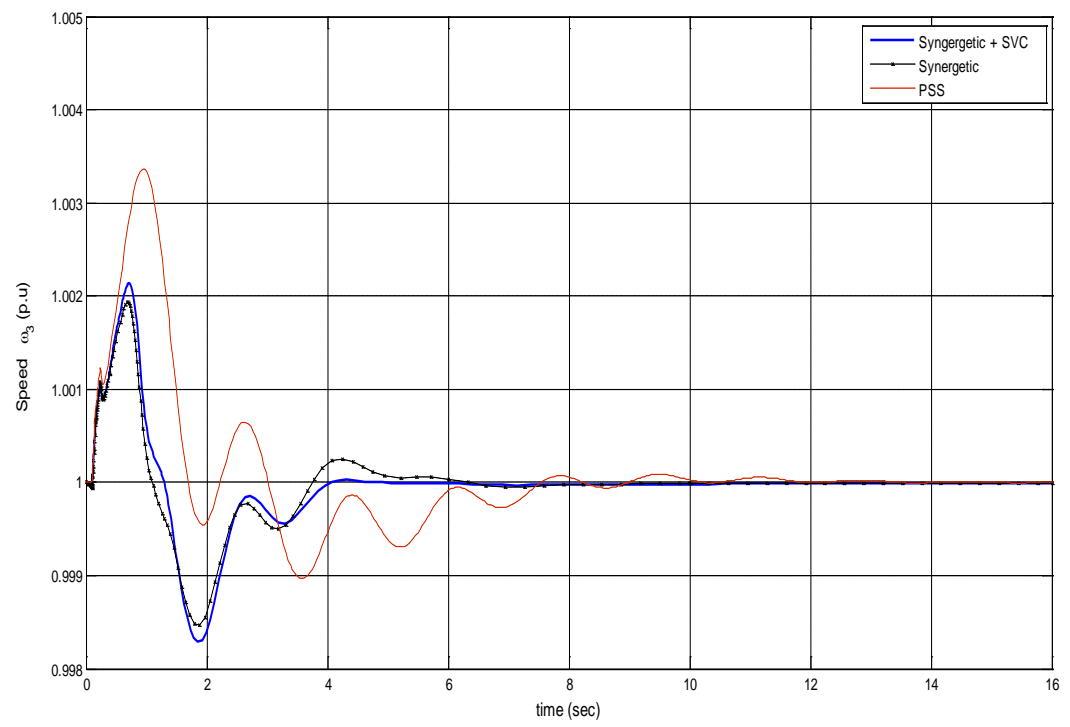

Figure 4.13: Angular speed of generator 3 


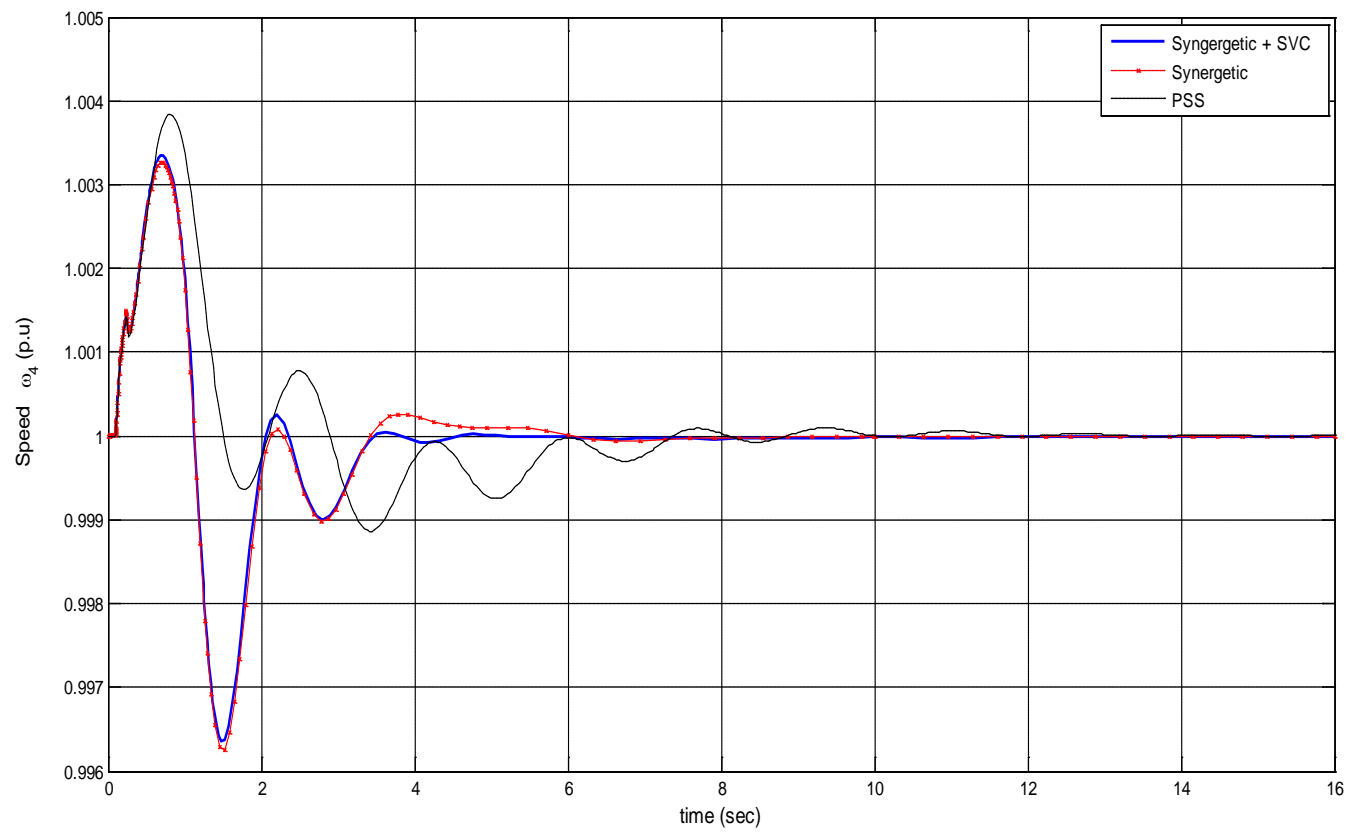

Figure 4.14: Angular speed of generator 4

In summary, simultaneous tuning of decentralized synergetic controllers and SVC supplementary controller using PSO algorithm is proposed in this chapter. For each of the machine, a decentralized synergetic controller is explicitly designed. A lead lag compensator is employed as the SVC supplementary controller. The interaction of each machine with the rest of the system is modeled locally and treated as external disturbance to it. The method is tested successfully on a SMIB-FACT system and on a two area four machines system with the SVC included in the middle of the lines connecting the two areas together. The results, when compared with the previous works $[47,16]$, give better performance. 


\section{Chapter 5}

\section{Decentralized Synergetic Control with Variable Structure}

\subsection{Reinforcement Learning}

In this chapter, decentralized synergetic controller with variable parameters is proposed to improve on the performance obtained when a fixed control structure is employed in chapter 2 . Synergetic control design techniques have been applied and tested to give better performance than conventional power system stabilizers [33, 47, 48]. In [33], the technique was employed to design a nonlinear control for a Single Machine Infinite Bus (SMIB) system. In [47], decentralized synergetic controllers were designed by the authors to dampen power oscillations. The controller's outputs are fed as a supplementary signal to the exciter of the generator.

In [48], decentralized synergetic controllers are coordinated with SVC-damping controllers. PSO technique is employed in both $[47,48]$ to obtain optimal value for each of the controller's parameters. In this paper, PSO is chosen to select optimal values of the controller parameters and $\mathrm{RL}$ is added so as to improve the performance of the controllers for a wide range of the system's operating conditions. PSO technique is selected over other computational intelligent algorithms like Genetic Algorithms (GA) because of its computational efficiency, robustness and its simplicity of implementation $[49,44]$. The RL tool is employed to vary some of the parameters of the controllers.

Some previous work on the application of RL methods in power system stability improvement are reported in [19], [20], [50], [7], [8], [25]. In [19, 20], RL was applied to adjust the gains of the conventional PSS, which is based on linearized model of the system. In [50], and [7], it was applied to dynamic brake controllers and thyristor controlled series capacitors (TCSC), respectively. The 
method is also applied in [8] for load frequency control and in [25], for power oscillation control by adjusting the gains of adaptive decentralized backstepping controllers. Although the primary controllers, the adaptive backstepping controllers, are decentralized, the RL is not. This makes the entire algorithms, RL and backstepping controllers, not completely decentralized. In this paper, a completely decentralized algorithm is employed. Both the synergetic controllers and the RL are decentralized.

In this dissertation, the Q- learning type of RL is implemented. This is simply because of its independence on the model of the system, and its suitability for step-by-step incremental computation. In general, any "agent" that utilizes RL technique tries to learn behavior by interacting with its environment in order to annex experience and learn to act in an optimal way. This learning is done iteratively. RL assumes that the environment is divided into a finite state and described by a set of state $\{S\}$.At each step, the "agent" observes the current state $s_{t}$ of the system, and selects an action $a$ from a set of actions $\{A\}$ at time $t$. The agent is rewarded immediately after taken this action. This reward $r_{t+1}$ is given from a set of rewards $\{R\}$, and it quantifies the effectiveness of the action taken. The action will affect the environment and takes it to a new state $s_{t+1}$. The interaction between the agent and the environment is repeated until the desired goal is accomplished. The goal of RL is to find a policy, a matching between states and actions, which will result in maximum expected discounted long- term reward or return. The expected discounted return is given as:

$$
R_{t}=\sum_{k=0}^{\infty} \gamma^{k} r_{t+k+1}
$$

The variable $\gamma$, whose value is between 0 and 1 , is known as discounted rate. It indicates the significance of the future reward in the decision making process. If the value is set to 0 , only the immediate reward is taken into consideration by the agent, but when the value is a 1 , all the future rewards are put into account. Each RL comprises of two major functions: the state value function, which is the expected return when starting at state $s_{t}$ using policy $\pi(s, a)$; and the action value function, which is the expected discounted return when starting at state $s_{t}$ taken action $a_{t}$ and using policy $\pi(s, a)$. It is also known as $\mathrm{Q}$-function and its value is given by the following equation: 


$$
Q^{\pi}(s, a)=E_{\pi}\left\{\sum_{k=0}^{\infty} \gamma^{k} r_{t+1+k} \mid s_{t}=s, a_{t}=a\right\} .
$$

The RL method tries to find the optimal policy $\pi^{*}$ that will maximize the action function (5.2). One of the algorithms that can be employed is the Q-learning algorithms. It is model independent. It gains experience by interacting with the environment to update the value function, (5.2), at every time step. The update is done with the bellman optimality equation which is given as:

$$
\Delta Q=\alpha\left[r_{t+1}+\gamma \max Q\left(s_{t+1}, a_{t+1}\right)-Q\left(s_{t}, a_{t}\right)\right]
$$

Where $\alpha,(0<\alpha<1)$ is a constant step-size that denotes the amount of corrected error. The steps of the Q-learning algorithm are summarized below:

- Initialize $Q(s, a)$ for all the states and actions

- Repeat for each run of the algorithm

Initialize $s$. Repeat for each step

Take action $a$ based on policy determine by $Q^{1}$.

Observe $s_{t+1}$ and $r$.

Modify Q based on (5.3).

$$
S=S_{t+1}
$$

- $\quad$ Repeat until the desired goal is achieved or the terminal state is reached.

In this paper, the RL is employed to adjust or vary parameters $\lambda_{i}$ and $K_{i}$ of the synergetic controller of each sub-system. The state of RL is the Integral Time Absolute Error (ITAE) given in (5.4). The update of the action value of each sub-system is done every $0.5 s$ and the action is taken by increasing or decreasing the values $\lambda_{i}, K_{i}$ by a value corresponding to the absolute value of the active power deviation at that time step. 
$J_{i}=\int_{t_{0}}^{t}\left[\kappa_{1}\left|\Delta P_{e i}\right|+\kappa_{2}\left|\Delta \omega_{i}\right|+\kappa_{3}\left|\Delta V_{T i}\right|\right] d t$

Where $\kappa_{1}, \kappa_{2}$ and $\kappa_{3}$ are weighing values and are given as 200, 500 and 10 respectively. The immediate reward is given as a function of the state as:

Reward $=-50 \mathrm{~J}_{\mathrm{i}}$

The proposed technique is implemented on a two area system. The simulation results are obtained using PAT [46]. The results are presented in the next section.

\subsection{Problem Formulation}

The combination of RL and synergetic control is employed to dampen power system oscillations through the excitation of the generator. In a multimachine system, each generator is considered as a decoupled sub-system, and a decentralized controller is designed for each sub-system. The coupling effect of the rest of the system on the sub-system is considered as an external disturbance to the sub-system. It is estimated as a quadratic function of the active power generated by sub-system. PSO technique is utilized to initialize the value of the parameters of the controller. These initial values are considered to be the nominal values of the parameters. Then, RL is employed at higher level to vary some nominal parameter values of the controller within a certain range. The effect of $\mathrm{RL}$ is to adapt the controller to varying operating conditions and uncertainties of the external disturbance.

\subsection{1 $i^{\text {th }}$ subsystem model}

Each sub-system is modeled by a third -order single axis modeled. The state space model of the $i^{\text {th }}$ sub-system is given by the following equations:

$$
\begin{gathered}
\Delta \dot{\delta}_{i}=\omega_{o} \Delta \omega_{i} \\
\Delta \dot{\omega}_{i}=-\frac{D_{i}}{2 H_{i}} \Delta \omega_{i}-\frac{1}{2 H_{i}} \Delta P_{e i}
\end{gathered}
$$


$\Delta \dot{P}_{e i}=-\frac{1}{T_{d o i}^{\prime}} \Delta P_{e i}+\frac{1}{T_{d o i}^{\prime}} v_{f i}+d_{i}$

Where:

$v_{f i}=k_{c i} I_{q i} u_{f i}-\left(x_{d i}-x_{d i}^{\prime}\right) I_{d i} I_{q i}-P_{m i o}-T_{d o i}^{\prime} Q_{e i} \omega_{o} \Delta \omega_{i}$

$\mathrm{d}_{\mathrm{i}}=\mathrm{E}_{\mathrm{qi}}^{\prime} \sum_{\mathrm{j}=1}^{\mathrm{n}} \dot{\mathrm{E}}_{\mathrm{qj}}^{\prime} \mathrm{B}_{\mathrm{ij}} \sin \left(\delta_{\mathrm{i}}-\delta_{\mathrm{j}}\right)-\mathrm{E}_{\mathrm{qi}}^{\prime} \sum_{\mathrm{j}=1}^{\mathrm{n}} \mathrm{E}_{\mathrm{qj}}^{\prime} \mathrm{B}_{\mathrm{ij}} \cos \left(\delta_{\mathrm{i}}-\delta_{\mathrm{j}}\right)\left(\omega_{\mathrm{j}}\right)$

$d_{i}$ is the coupling term that connects the $i$ th sub-system to the rest of the system and $\Delta$ denotes deviation from the nominal state.

\subsubsection{Controller Design}

The objectives are to define a stable and invariant manifold, and to come up with a control that will steer the system trajectories and forces them to remain on this manifold. Given a sub-system described by (5.6), (5.7) and (5.8), a macro-variable is defined, using the procedure of Slotine and Li [37], as follow:

$\varphi_{i}=\left(\frac{d}{d t}+\lambda_{i}\right)^{2} \Delta \delta_{i}$

For the manifold, $\varphi_{i}=0$, that will evolve from (5.11) to be stable, $\lambda_{i}>0$. By substituting (5.6) and (5.7) into (5.11), the macro-variable can be re-written as:

$\varphi_{i}=-\frac{\omega_{o}}{2 H_{i}} \Delta P_{e i}+\omega_{o}\left(2 \lambda_{i}-\frac{D_{i}}{2 H_{i}}\right) \Delta \omega_{i}+\lambda_{i}^{2} \Delta \delta_{i}$

The dynamic of the evolution of the macro-variable for the $i$ th sub-system is given as: 


$$
K_{i} \dot{\varphi}_{i}+\varphi_{i}=0
$$

Denoting $\Delta \delta_{i}, \Delta \omega_{i}$ and $\Delta P_{e i}$ by $x_{1 i}, x_{2 i}$ and $x_{3 i}$ respectively and by substituting (11) and its derivative into (12) we can obtain:

$-\frac{\omega_{o}}{2 H_{i}} \dot{x}_{3 i}+\omega_{o}\left(2 \lambda_{i}-\frac{D_{i}}{2 H_{i}}\right) \dot{X}_{2 i}+\lambda_{i}^{2} \dot{X}_{1 i}=-\frac{\omega_{o}}{K_{i}}\left(-\frac{1}{2 H_{i}} X_{3 i}+\left(2 \lambda_{i}-\frac{D_{i}}{2 H_{i}}\right) X_{2 i}+\frac{\lambda_{i}^{2}}{\omega_{o}} X_{1 i}\right)$

$$
\begin{aligned}
& -\frac{\omega_{o}}{2 H_{i}} \dot{X}_{3 i}=-\omega_{o}\left\{\left(2 \lambda_{i}-\frac{D_{i}}{2 H_{i}}\right)\left(-\frac{D_{i}}{2 H_{i}} x_{2 i}-\frac{1}{2 H_{i}} X_{3 i}\right)\right. \\
& \left.\quad+\lambda_{i}^{2} x_{2 i}+\frac{1}{K_{i}}\left[-\frac{1}{2 H_{i}} X_{3 i}+\left(2 \lambda_{i}-\frac{D_{i}}{2 H_{i}}\right) x_{2 i}+\frac{\lambda_{i}^{2}}{\omega_{o}} X_{1 i}\right]\right\}
\end{aligned}
$$

$$
\frac{1}{2 \mathrm{H}_{\mathrm{i}}} \dot{\mathrm{x}}_{3 \mathrm{i}}=\left\{\left[\left(2 \lambda_{\mathrm{i}}-\frac{\mathrm{D}_{\mathrm{i}}}{2 \mathrm{H}_{\mathrm{i}}}\right)\left(\frac{1}{\mathrm{~K}_{\mathrm{i}}}-\frac{\mathrm{D}_{\mathrm{i}}}{2 \mathrm{H}_{\mathrm{i}}}\right)+\lambda_{\mathrm{i}}^{2}\right] \mathrm{x}_{2 \mathrm{i}}+\frac{\lambda_{\mathrm{i}}^{2}}{\omega_{\mathrm{o}} \mathrm{K}_{\mathrm{i}}} \mathrm{x}_{1 \mathrm{i}}-\frac{1}{2 \mathrm{H}_{\mathrm{i}}}\left(2 \lambda_{\mathrm{i}}-\frac{\mathrm{D}_{\mathrm{i}}}{2 \mathrm{H}_{\mathrm{i}}}+\frac{1}{\mathrm{~K}_{\mathrm{i}}}\right) \mathrm{x}_{3 \mathrm{i}}\right\}
$$

This gives:

$$
\dot{\mathrm{x}}_{3 \mathrm{i}}=\frac{2 \mathrm{H}_{\mathrm{i}} \lambda_{\mathrm{i}}^{2}}{\omega_{\mathrm{o}} \mathrm{K}_{\mathrm{i}}} \mathrm{x}_{1 \mathrm{i}}+2 \mathrm{H}_{\mathrm{i}}\left\{\lambda_{\mathrm{i}}^{2}+\left(2 \lambda_{\mathrm{i}}-\frac{\mathrm{D}_{\mathrm{i}}}{2 \mathrm{H}_{\mathrm{i}}}\right)\left(\frac{1}{\mathrm{~K}_{\mathrm{i}}}-\frac{\mathrm{D}_{\mathrm{i}}}{2 \mathrm{H}_{\mathrm{i}}}\right)\right\} \mathrm{x}_{2 \mathrm{i}}-\left(2 \lambda_{\mathrm{i}}-\frac{\mathrm{D}_{\mathrm{i}}}{2 \mathrm{H}_{\mathrm{i}}}+\frac{1}{\mathrm{~K}_{\mathrm{i}}}\right) \mathrm{x}_{3 \mathrm{i}}
$$

By substituting (5.8) into (5.17) we can obtain

$v_{f i}+T_{d o i}^{\prime} d_{i}=\frac{2 H_{i} T_{d o}^{\prime} \lambda_{i}^{2}}{\omega_{0} K_{i}} X_{1 i}+2 H_{i} T_{d o i}^{\prime}\left\{\lambda_{i}^{2}+\left(2 \lambda_{i}-\frac{D_{i}}{2 H_{i}}\right)\left(\frac{1}{K_{i}}-\frac{D_{i}}{2 H_{i}}\right)\right\} x_{2 i}+\left\{1-T_{d o i}^{\prime}\left(2 \lambda_{i}-\frac{D_{i}}{2 H_{i}}+\frac{1}{K_{i}}\right)\right\} x_{3 i}$ 
Assuming that the coupling term $d_{i}$ can be estimated by equation (18) where $c_{1 i}$ and $c_{2 i}$ are two unknown constant parameters:

$$
d_{i} \approx c_{1 i} x_{3 i}+c_{2 i} x_{3 i}^{2}
$$

The method of adaptation can be employed to approximate this coupling term. Let us define $\hat{d}_{i}=\hat{c}_{1 i} x_{3 i}+\hat{c}_{2 i} x_{3 i}^{2}$, where $\hat{c}_{1 i}$ and $\hat{c}_{2 i}$ are the estimate values of $c_{1 i}$ and $c_{2 i}$ respectively. To obtain these values, we define a lyapunov function as follow:

$V_{i}=\frac{1}{2} \varphi_{i}^{2}+\frac{1}{2}\left(c_{1 i}-\hat{c}_{1 i}\right)^{2} \Gamma_{1 i}^{-1}+\frac{1}{2}\left(c_{2 i}-\hat{c}_{2 i}\right)^{2} \Gamma_{2 i}^{-1}$

where $\Gamma_{1 i}$ and $\Gamma_{2 i}$ are adaptation gains. Differentiating (5.20), we have:

$\dot{V}_{i}=\varphi_{i} \dot{\varphi}_{i}+\left(c_{1 i}-\hat{c}_{1 i}\right) \Gamma_{1 i}^{-1} \dot{\hat{c}}_{1 i}+\left(c_{2 i}-\hat{c}_{2 i}\right) \Gamma_{2 i}^{-1} \dot{\hat{c}}_{2 i}$

Let define

$\dot{\hat{c}}_{1 i}=\Omega_{1 i} \varphi_{i} x_{3 i}$

$\dot{\hat{c}}_{2 i}=\Omega_{2 i} \varphi_{i} x_{3 i}^{2}$

Where $\Omega_{1 i}=\frac{\omega_{o}}{2 H_{i}} \Gamma_{1 i}$ and $\Omega_{2 i}=\frac{\omega_{o}}{2 H_{i}} \Gamma_{2 i}$. By substituting (5.22) and (5.23) into (5.21) and solving for $v_{f i}$ we have:

$v_{f i}=\frac{2 H_{i} T_{d o i}^{\prime} \lambda_{i}^{2}}{\omega_{o} K_{i}} x_{1 i}+2 H_{i} T_{d o i}^{\prime}\left\{\lambda_{i}^{2}+\left(2 \lambda_{i}-\frac{D_{i}}{2 H_{i}}\right)\left(\frac{1}{K_{i}}-\frac{D_{i}}{2 H_{i}}\right)\right\} x_{2 i}+\left\{1-T_{d o i}^{\prime}\left(2 \lambda_{i}-\frac{D_{i}}{2 H_{i}}+\frac{1}{K_{i}}\right)\right\} x_{3 i}-T_{d o i}^{\prime} \hat{d}_{i}$ 
By substituting (5.22), (5.23) and the derivative of (5.12) into (5.21) we have

$$
\dot{V}_{i}=\varphi_{i}\left(-\frac{\omega_{o}}{2 H_{i}} \dot{x}_{3 i}+\omega_{o}\left(2 \lambda_{i}-\frac{D_{i}}{2 H_{i}}\right) \dot{x}_{2 i}+\lambda_{i}^{2} \dot{x}_{1 i}\right)+\left(c_{1 i}-\hat{c}_{1 i}\right) \Gamma_{1 i}^{-1}\left(\Omega_{1 i} \varphi_{i} x_{3 i}\right)+\left(c_{2 i}-\hat{c}_{2 i}\right) \Gamma_{2 i}^{-1}\left(\Omega_{2 i} \varphi_{i} x_{3 i}^{2}\right)
$$

$$
\begin{aligned}
\dot{V}_{i}= & \omega_{o} \varphi_{i}\left\{\frac{1}{2 H T_{d o i}^{\prime}}\left(x_{3 i}-v_{f i}-T_{d o i}^{\prime} d_{i}\right)+\left(2 \lambda_{i}-\frac{D_{i}}{2 H_{i}}\right)\left(-\frac{D_{i}}{2 H_{i}} x_{2 i}-\frac{1}{2 H_{i}} x_{3 i}\right)+\lambda_{i}^{2} x_{2 i}\right. \\
& \left.+\frac{1}{2 H_{i}}\left(c_{1 i}-\hat{c}_{1 i}\right) x_{3 i}+\frac{1}{2 H_{i}}\left(c_{2 i}-\hat{c}_{2 i}\right) x_{3 i}^{2}\right\}
\end{aligned}
$$

$$
\begin{aligned}
\dot{V}_{i}= & \frac{\omega_{o} \varphi_{i}}{2 H_{i} T_{d o i}^{\prime}}\left\{x_{3 i}-v_{f i}-T_{d o i}^{\prime} d_{i}+2 H_{i} T_{d o i}^{\prime}\left(\lambda_{i}^{2}-\frac{D_{i}}{2 H_{i}}\left(2 \lambda_{i}-\frac{D_{i}}{2 H_{i}}\right)\right) x_{2 i}-T_{d o i}^{\prime}\left(2 \lambda_{i}-\frac{D_{i}}{2 H_{i}}\right) x_{3 i}\right. \\
& +T_{d o i}^{\prime}\left(c_{1 i} x_{3 i}+c_{2 i} x_{3 i}^{2}\right)-T_{d o i}^{\prime}\left(\hat{c}_{1 i} x_{3 i}+\hat{c}_{2 i} x_{3 i}^{2}\right) \\
\dot{V}_{i}= & \frac{\omega_{o} \varphi_{i}}{2 H_{i} T_{d o i}^{\prime}}\left\{-v_{f i}-T_{d o i}^{\prime} d_{i}+2 H_{i} T_{d o i}^{\prime}\left(\lambda_{i}^{2}-\frac{D_{i}}{2 H_{i}}\left(2 \lambda_{i}-\frac{D_{i}}{2 H_{i}}\right)\right) x_{2 i}+\left(1-T_{d o i}^{\prime}\left(2 \lambda_{i}-\frac{D_{i}}{2 H_{i}}\right)\right) x_{3 i}\right. \\
& +T_{d o i}^{\prime} d_{i}-T_{d o i}^{\prime} \hat{d}_{i}
\end{aligned}
$$

By substituting (5.24) into (5.28) we have

$$
\begin{aligned}
\dot{V}_{i}= & \frac{\omega_{o} \varphi_{i}}{2 H_{i} T_{d o i}^{\prime}}\left\{-\frac{2 H_{i} T_{d o i}^{\prime} \lambda_{i}^{2}}{\omega_{o} K_{i}} x_{1 i}-2 H_{i} T_{d o i}^{\prime}\left(\lambda_{i}^{2}-\frac{D_{i}}{2 H_{i}}\left(2 \lambda_{i}-\frac{D_{i}}{2 H_{i}}\right)\right) x_{2 i}-\frac{2 H_{i} T_{d o i}^{\prime}}{K_{i}}\left(2 \lambda_{i}-\frac{D_{i}}{2 H_{i}}\right) x_{2 i}+T_{d o i}^{\prime} \hat{d}_{i}\right. \\
& -\left(1-T_{d o i}^{\prime}\left(2 \lambda_{i}-\frac{D_{i}}{2 H_{i}}\right)\right) x_{3 i}+\frac{T_{d o i}^{\prime}}{K_{i}} X_{3 i}+2 H_{i} T_{d o i}^{\prime}\left(\lambda_{i}^{2}-\frac{D_{i}}{2 H_{i}}\left(2 \lambda_{i}-\frac{D_{i}}{2 H_{i}}\right)\right) x_{2 i}+\left(1-T_{d o i}^{\prime}\left(2 \lambda_{i}-\frac{D_{i}}{2 H_{i}}\right)\right) x_{3 i} \\
& \left.-T_{d o i}^{\prime} \hat{d}_{i}\right\}
\end{aligned}
$$

$\dot{V}_{i}=-\frac{\varphi_{i}}{K_{i}}\left(-\frac{\omega_{o}}{2 H_{i}} x_{3 i}+\omega_{o}\left(2 \lambda_{i}-\frac{D_{i}}{2 H_{i}}\right) x_{2 i}+\lambda_{i}^{2} x_{1 i}\right)=-\frac{1}{K_{i}} \varphi_{i}^{2}<0$

Hence, the system is guaranteed to be stable. The controller output $u_{f i}$ can be obtained from (5.9) and (5.24) as: 


$$
\begin{aligned}
u_{f i}= & \frac{1}{k_{c i} I_{q i}}\left\{P_{m i}+\left(x_{d i}-x_{d i}^{\prime}\right) I_{d i} I_{q i}+T_{d o i}^{\prime} Q_{e i} \omega_{o} x_{2 i}+\frac{2 H_{i} T_{d o i}^{\prime} \lambda_{i}^{2}}{\omega_{o} K_{i}} x_{1 i}+\right. \\
& \left.2 H_{i} T_{d o i}^{\prime}\left\{\lambda_{i}^{2}+\left(2 \lambda_{i}-\frac{D_{i}}{2 H_{i}}\right)\left(\frac{1}{K_{i}}-\frac{D_{i}}{2 H_{i}}\right)\right\} x_{2 i}+\left(1-T_{d o i}^{\prime}\left(2 \lambda_{i}-\frac{D_{i}}{2 H_{i}}+\frac{1}{K_{i}}\right)\right) x_{3 i}-T_{d o i}^{\prime} \hat{d}_{i}\right\}
\end{aligned}
$$

In the next section, the method of setting the optimal values of the controller parameters is briefly discussed. The parameters of the controller to be set are $K_{i}$ that determines speed at which the system trajectories are forced onto the manifold, $\lambda_{i}$ that describes the characteristics of the system after its trajectories is forced onto the manifold, the adaptive gains $\Omega_{1 i}, \Omega_{2 i}$, and $k_{c i}$.

\subsection{Optimum Settings for Controllers}

This section presents a global tuning procedure for decentralized synergetic controllers using Particle Swarm Optimization algorithm [43]. The algorithm is originally developed for nonlinear optimization problems with continuous variable. Some of various applications of this technique in power systems are addressed by Lee and El-Sharkawi in [9]. The algorithm works by first creating a 'population' of random solutions (also known as candidate solutions or particles). Each candidate solution is assigned to a random velocity value.

During each iteration, several candidate solutions are randomly selected and simultaneously maintained in the search space. The fitness of each of these solutions is determined based on a predefined objective function. The velocity and position of each particle is updated by using the following equations:

$$
\begin{aligned}
& v_{j_{-} \text {new }}=v_{j}+q_{1} \operatorname{rand}\left(\text { pbest }_{\text {loc }_{-} j}-p_{j}\right)+q_{2} \operatorname{Rand}\left(\text { pbest }_{\text {glo }}-p_{j}\right) \\
& p_{j_{-} \text {new }}=p_{j}+v_{j_{-} \text {new }}
\end{aligned}
$$

The process of obtaining new position for each of the particles maintained within the search space will terminate when some stopping criteria is met. Some of the common stop conditions include: a number of iteration since the last update of the global best particle, or a predefined target fitness value. The fitness value we adopted in this paper is given by the following equation: 


$$
J_{i}=\int_{0}^{t}\left(k_{w i}\left(\Delta \omega_{i}\right)^{2}+k_{v i}\left(\Delta V_{T i}\right)^{2}+k_{p i}\left(\Delta P_{i e}\right)^{2}\right)
$$

Where $\Delta V_{T i}$ is deviation in the terminal voltage of the $i$ th sub-system, and $k_{w i}, k_{v i}$ and $k_{p i}$ are weighing factors. The problem is thus to optimize (minimize) (5.34) subjects to system and controller's parameters constraints.

\subsection{Case Study}

The four machine system model [3] is utilized to illustrate the effectiveness of the proposed control technique on excitation control. The one line diagram of the test system is depicted in Figure 5.1. All generators are equipped with static exciter (Table 5.1). Generators 2 and 3 are also equipped with decentralized synergetic controllers (supplementary controllers). Two area system configurations, with $400 \mathrm{MW}$ of power flowing from area 1 to area 2, were analyzed.

- Operating condition 1- System with two transmission line between buses 13 and 101

- Operating condition 2- System with single line between buses 13 and 101

By considering the stability of these two conditions, the capability of the proposed controller to withstand a loss of a line after three phase fault at bus 3 is appraised. The results obtained are compared with fixed parameter synergetic controller tuned by particle swarm optimization and fixed gained linear power system stabilizer (PSS) tuned by genetic algorithm $[47,16]$.

The fixed parameter synergetic controller is tuned first based on PSO technique [3] to generate the optimum damping performance characteristic of the system for the particular contingency. Then reinforcement learning is employed to re-adjust some of the parameters of the controller under large disturbance at different operating points. 
Table 5.1: Parameters for Static Excitation

\begin{tabular}{c|c}
\hline Exciter & $1,2,3,4$ \\
\hline$K_{A}$ Regulator gain & 200 \\
\hline$T_{A}$ Regulator time constant (sec) & 0.05 \\
\hline$T_{R}$ Filter time constant (sec) & 0.01 \\
\hline$V_{R \max }$ Upper bound for saturation (p.u.) & 10 \\
\hline$V_{R \min }$ Lower bound for saturation (p.u.) & -10 \\
\hline
\end{tabular}

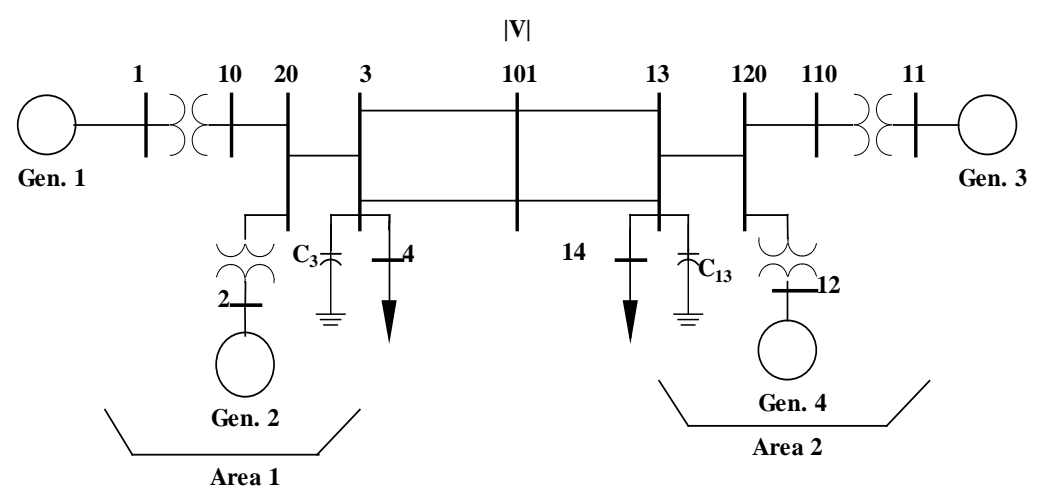

Figure 5.1: Two area system

The first scenario (operating condition 1 ) is three phase fault at time $t=0.1 \mathrm{sec}$, while the power is flowing from area 1 to area 2 . The near end of the line is opened and the line is removed at 0.19 sec. The line is reconnected back at $0.24 \mathrm{sec}$. The optimum parameters of the controllers are given in Table 2.

Table 5.2: Optimum parameters of the controllers

\begin{tabular}{llllll}
\hline$i$ & $\lambda_{i}$ & $K_{i}$ & $\Omega_{1 i}$ & $\Omega_{2 i}$ & $k_{c i}$ \\
2 & 21.95 & 0.578 & 32.74 & 31.75 & 3.341 \\
3 & 32.47 & 0.706 & 21.65 & 17.96 & 8.962 \\
\hline
\end{tabular}

Figures 5.2 and 5.3 depict the relative angles $\delta_{31}$ and $\delta_{41}$ respectively. The deviation in one of the tie-line power is depicted in Figure 5.5. The results delineate the superiority of the proposed 
reinforcement learning based synergetic control over the fixed parameter controller. Adjustment of the controllers' parameters $K_{i}$ and $\lambda_{i}$ for $i=2,3$ are shown in Figures 5.5 and 5.6.

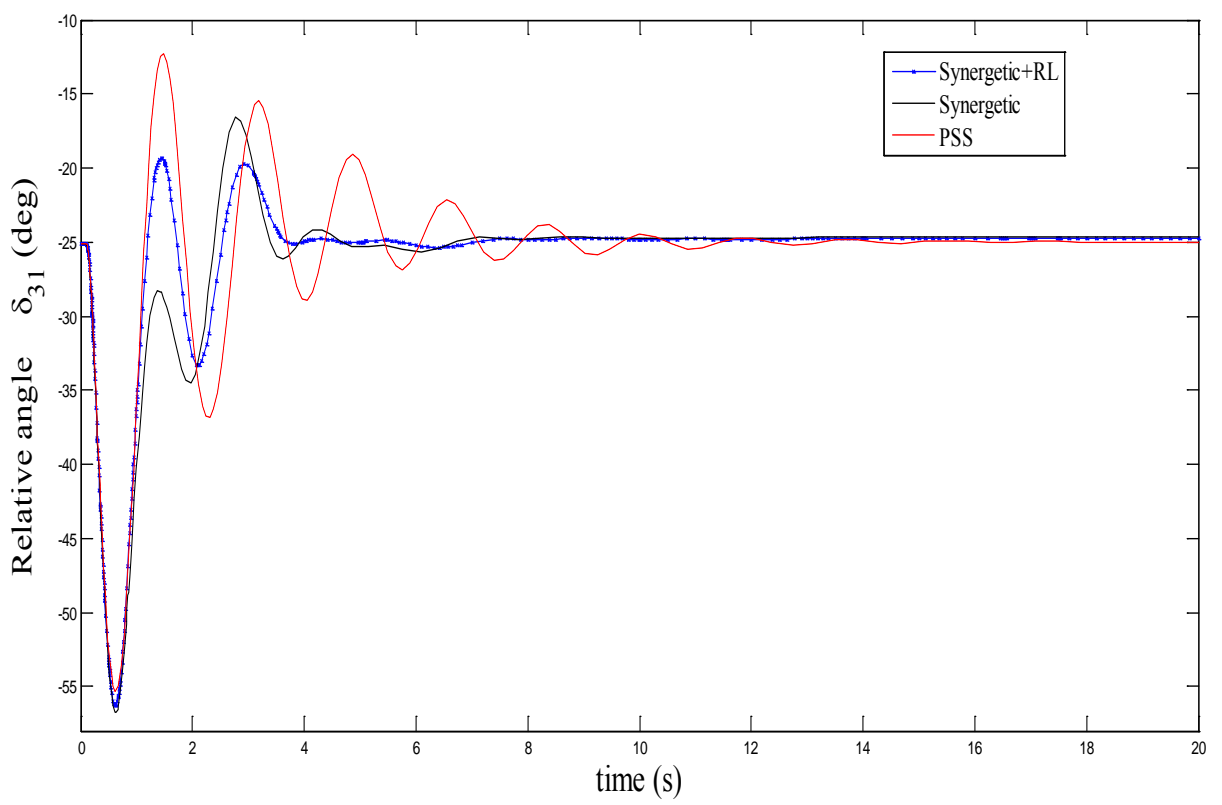

Figure 5.2: Relative angle $\delta_{31}$ under Operating Condition I

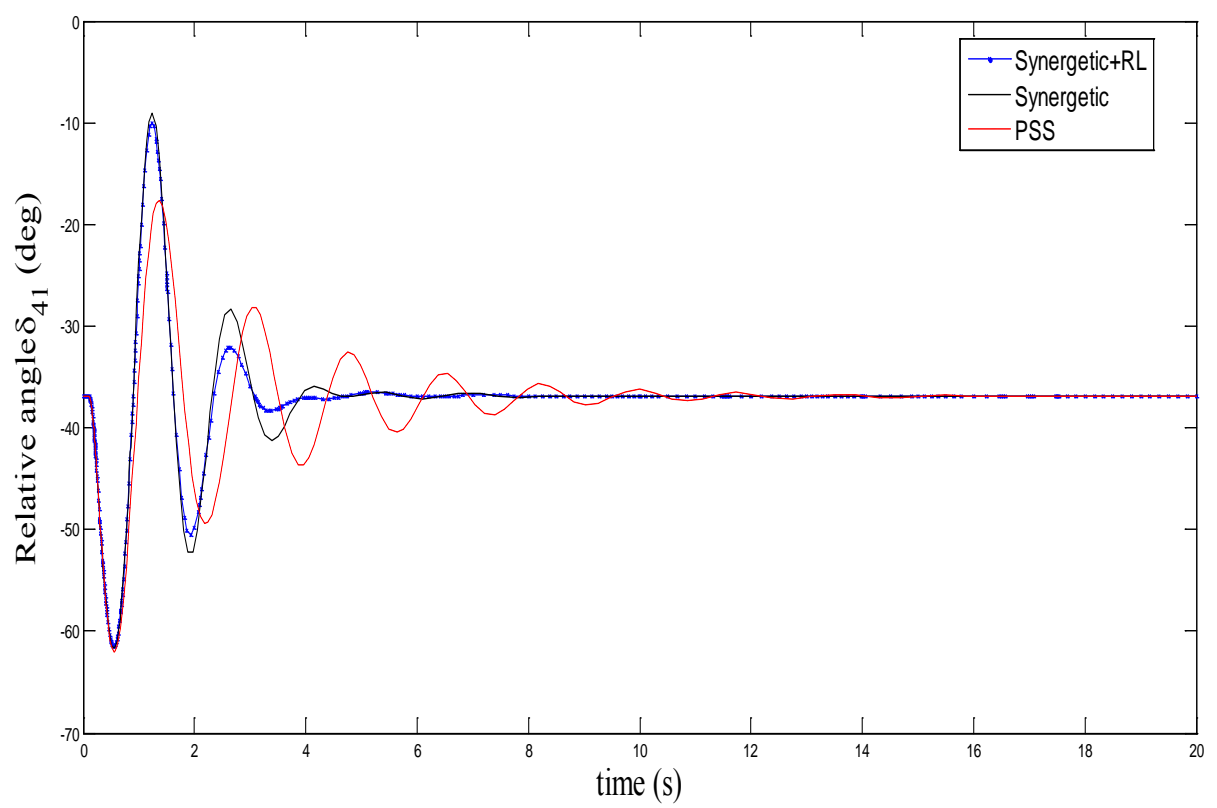

Figure 5.3: Relative angle $\delta_{41}$ under Operating Condition I 


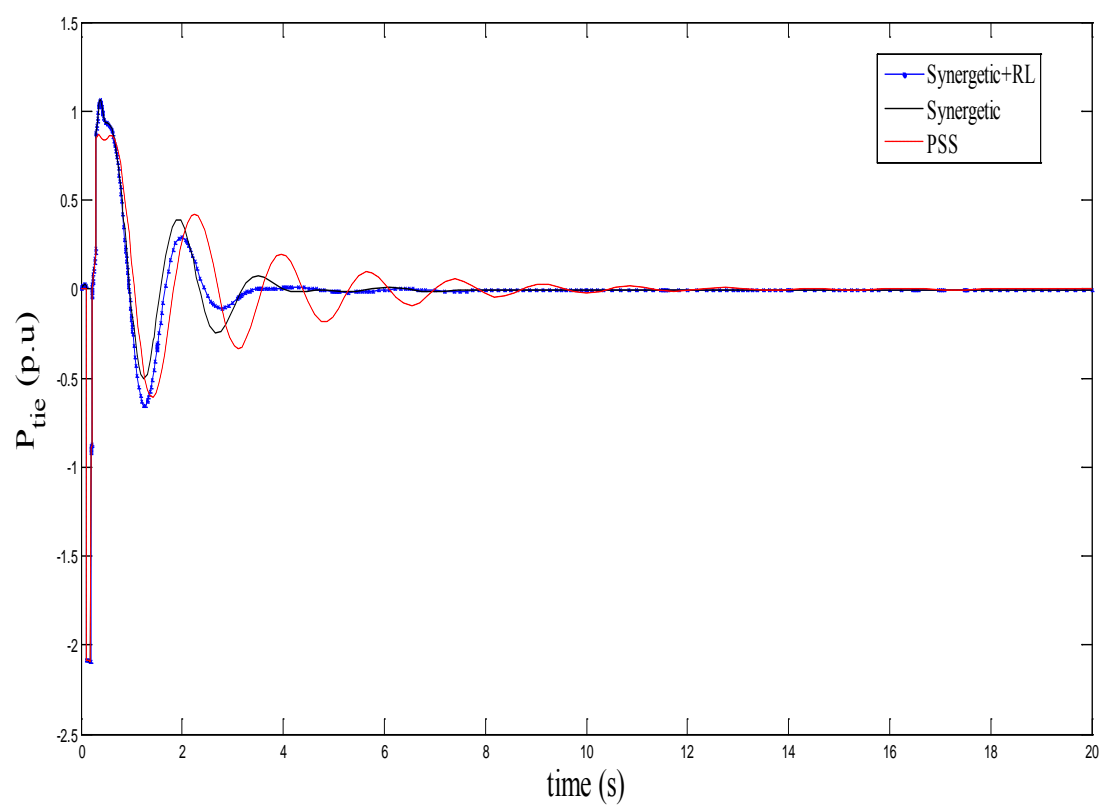

Figure 5.4: Tie line deviation under Operating Condition I
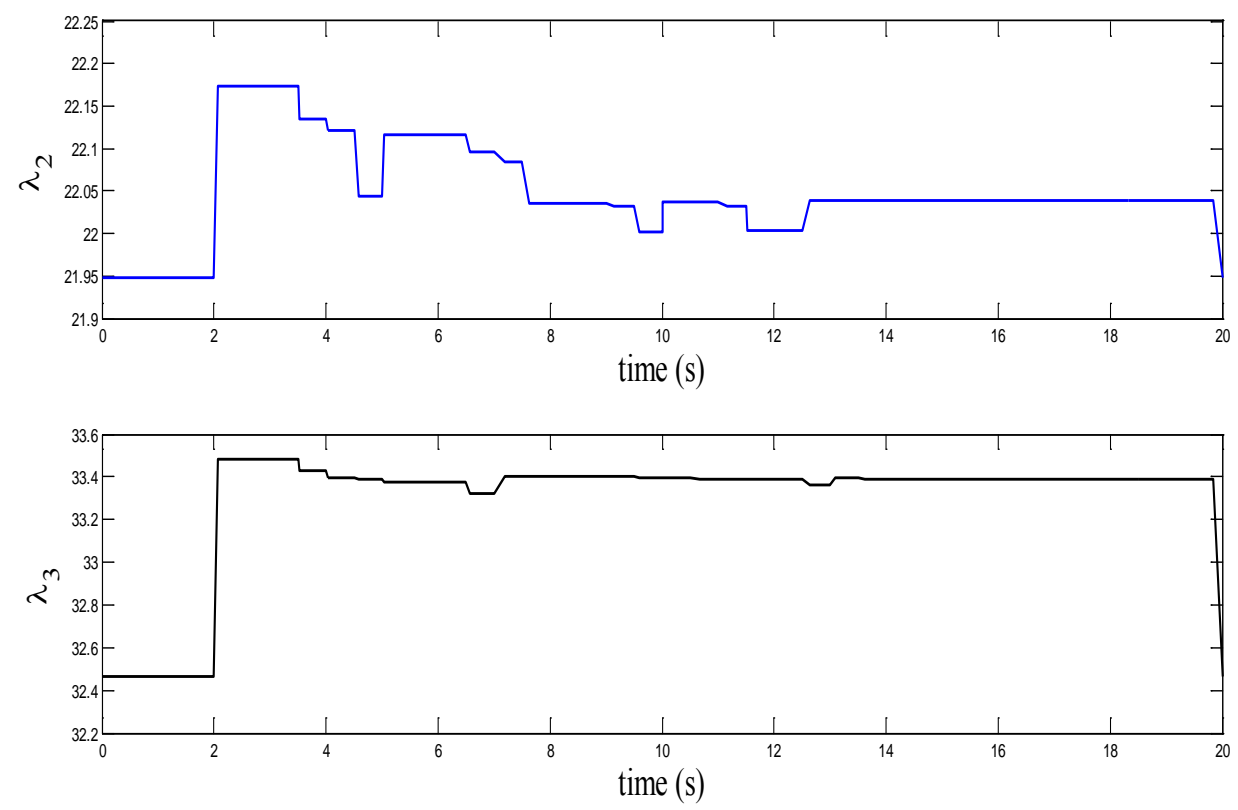

Figure 5.5: Operating Condition I, Parameters $\lambda_{2}, \lambda_{3}$ 


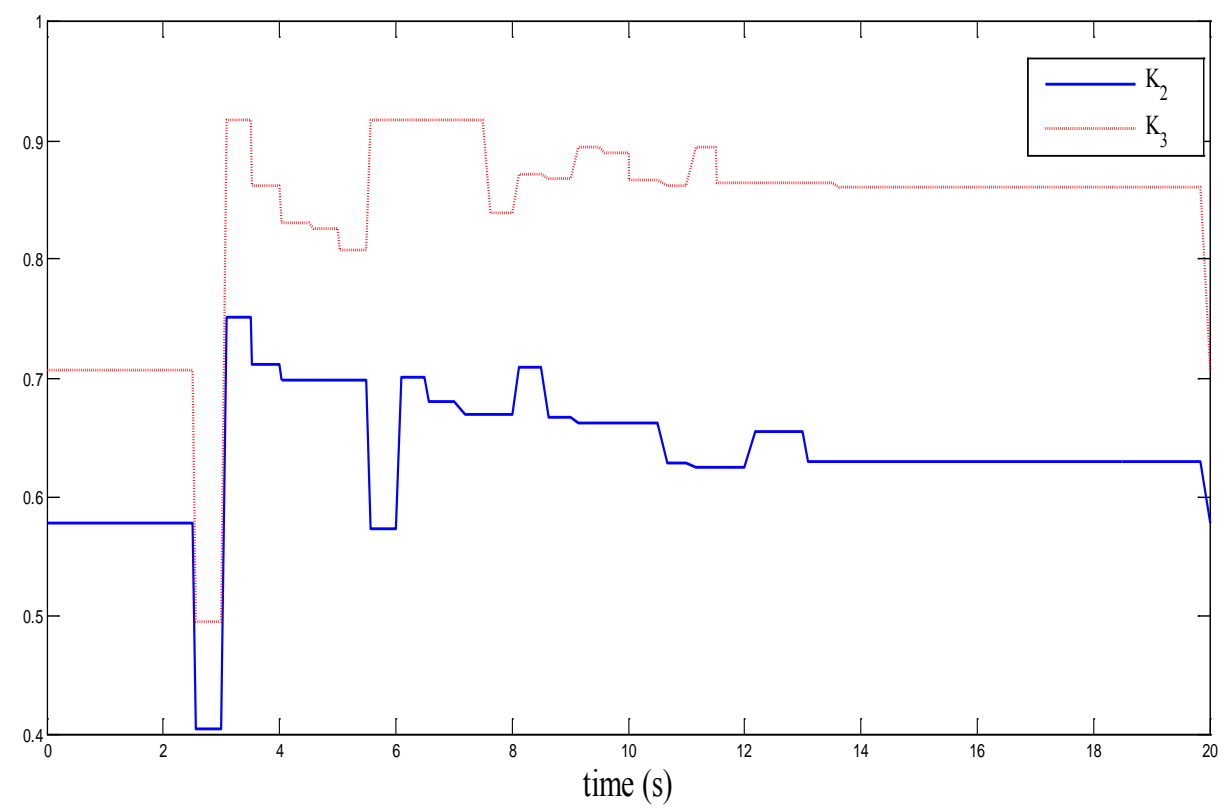

Figure 5.6: Operating Condition I, parameters $K_{2}, K_{3}$

Two scenarios are considered for the second operating condition. The first scenario is a three phase fault occurring on the line 3-101 at $t=0.1 \mathrm{~s}$. The near end of the fault is opened and the line is removed, clearing the fault at $t=0.23 \mathrm{~s}$. The line is reconnected at $t=0.26 \mathrm{~s}$. The same parameters given in Table 5.2 are utilized as the initial settings of the controllers. RL method is then employed to re-adjust parameters $\lambda_{i}, K_{i}$. The adjusted parameters $K_{i}$ are depicted in Figure 5.7, and the comparisons between relative angles $\delta_{31}, \delta_{41}$ are shown in Figures 5.8 and 5.9. The superiority of the proposed control techniques are also shown in these figures.

For the second scenario, the same fault occurs as in scenario 1, the fault is completely cleared at $t=0.26 \mathrm{~s}$, but the line not reconnected back. Figures 5.10 and 5.11 depict the comparison between the relative angles and we can see, from the figures, that the fixed PSS could not stabilize the system due to highly stressed transmission lines. The adjusted parameters are depicted in Figures 5.12 and 5.13 . 


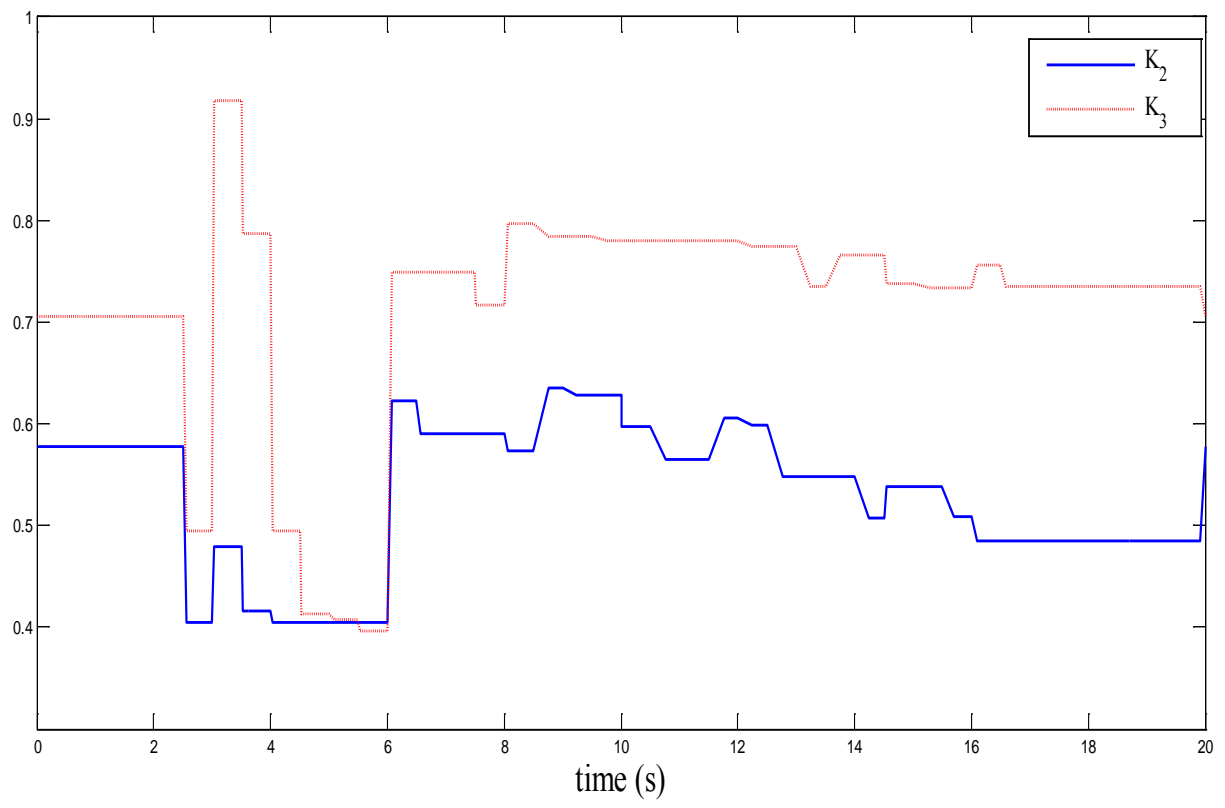

Figure 5. 7: Operating Condition II -Scenario I parameters $K_{2}, K_{3}$

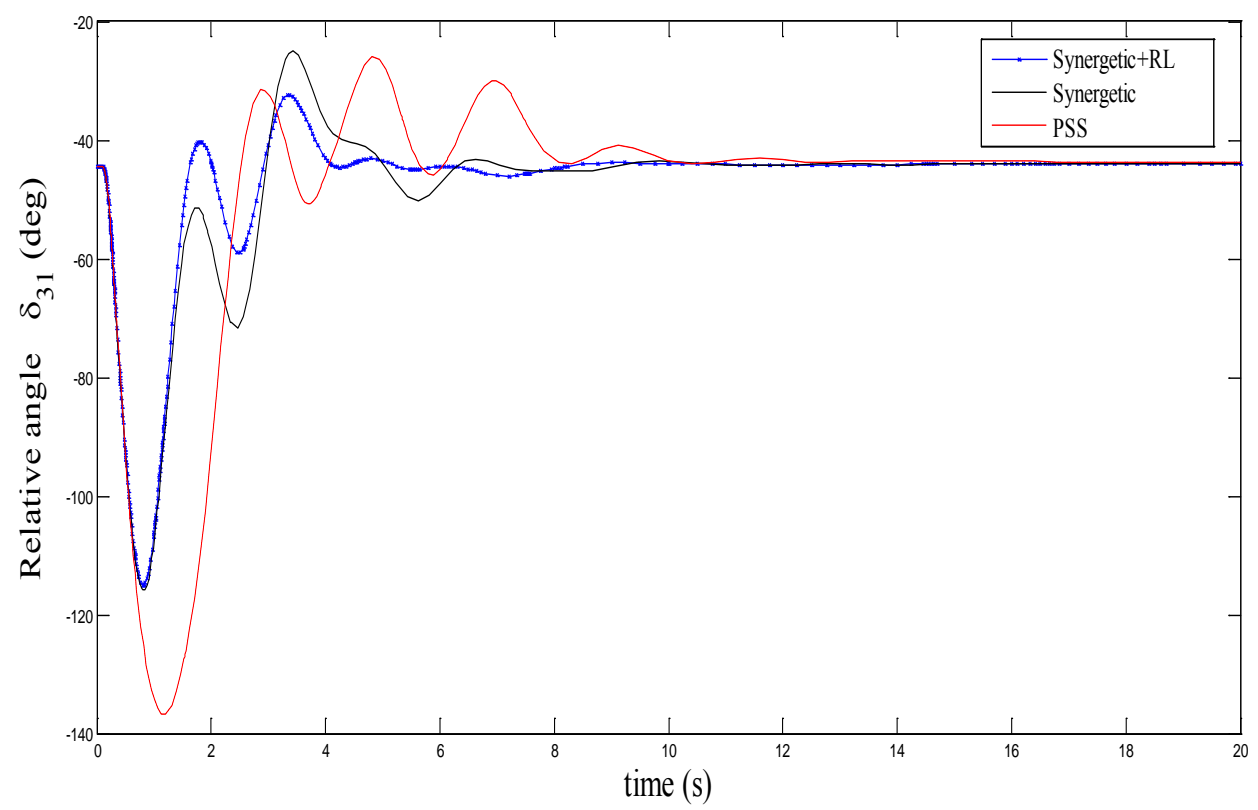

Figure 5.8: Relative angle $\delta_{31}$ under Operating Condition II Scenario I 


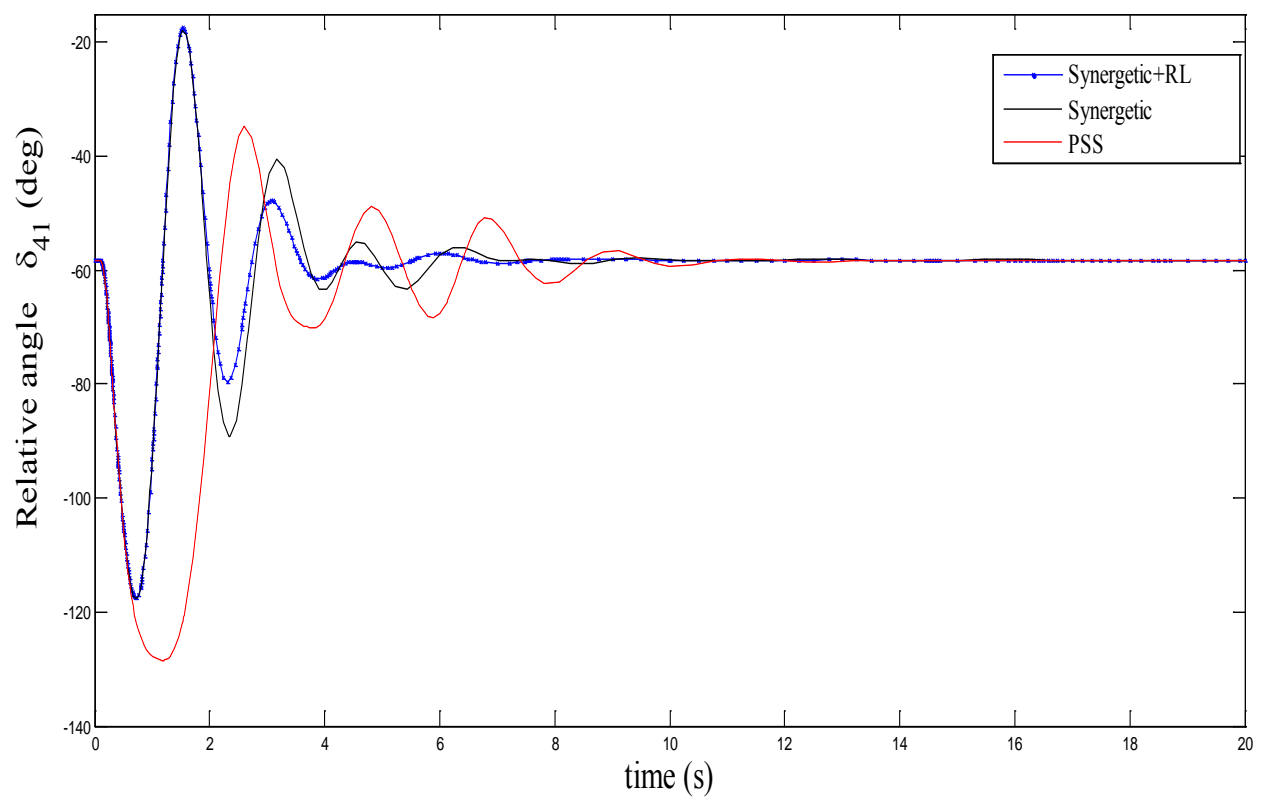

Figure 5.9: Relative angle $\delta_{41}$ under Operating Condition II Scenario I

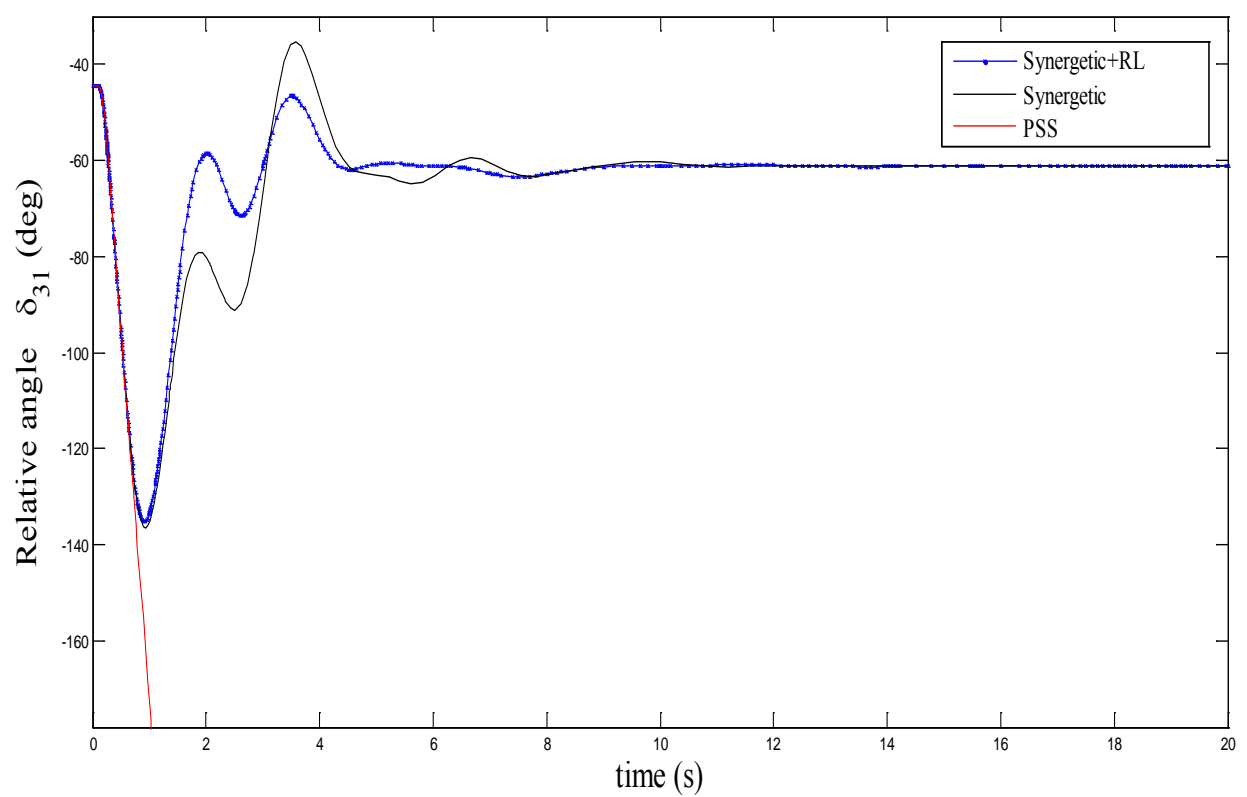

Figure 5.10: Relative angle $\delta_{31}$ under Operating Condition II Scenario II 


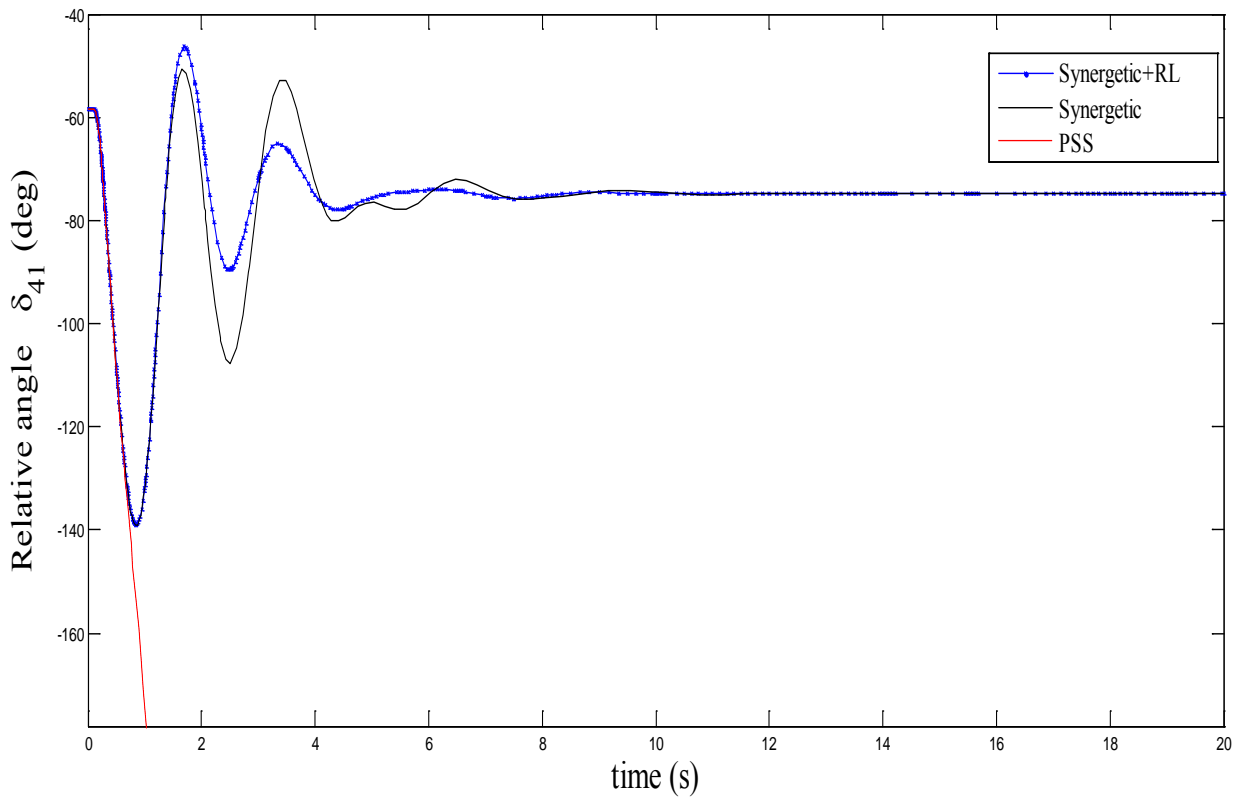

Figure 5.11: Relative angle $\delta_{41}$ under Operating Condition II Scenario I
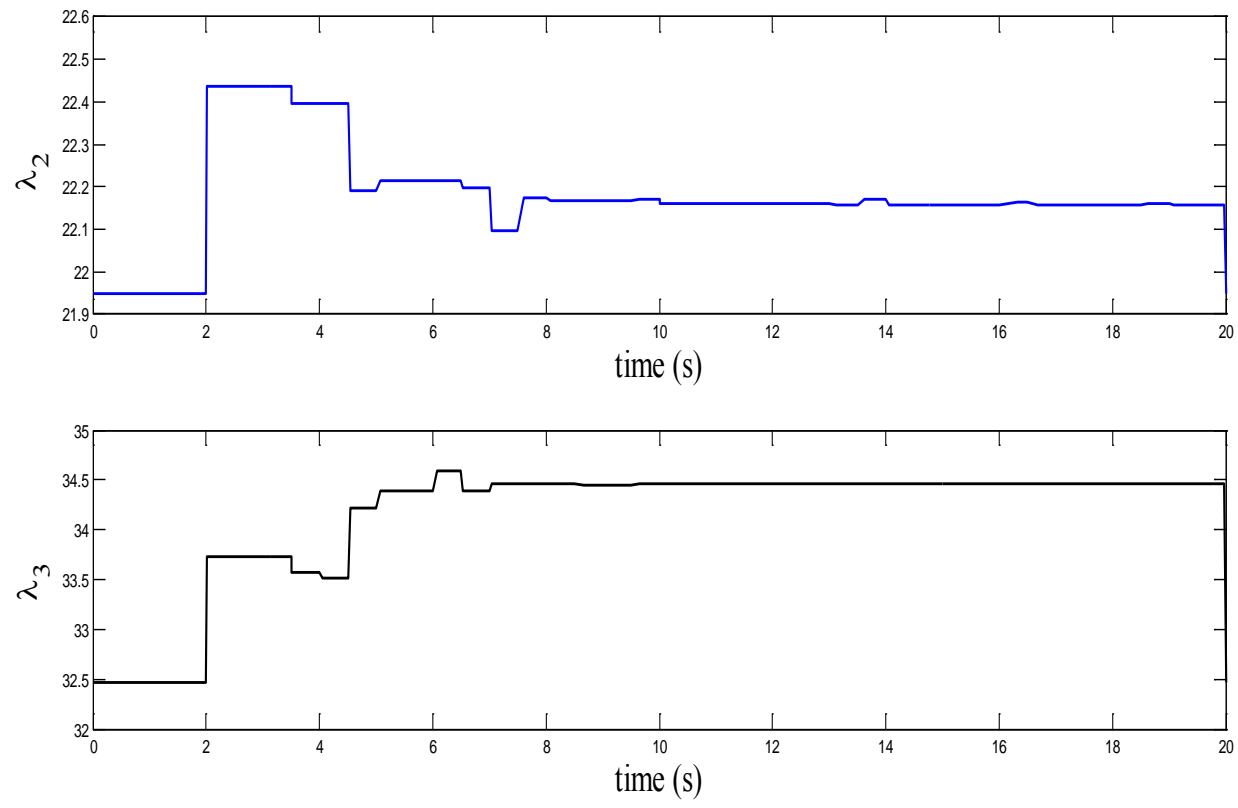

Figure 5.12: Operating Condition II-Scenario II, Parameters $\lambda_{2}, \lambda_{3}$ 


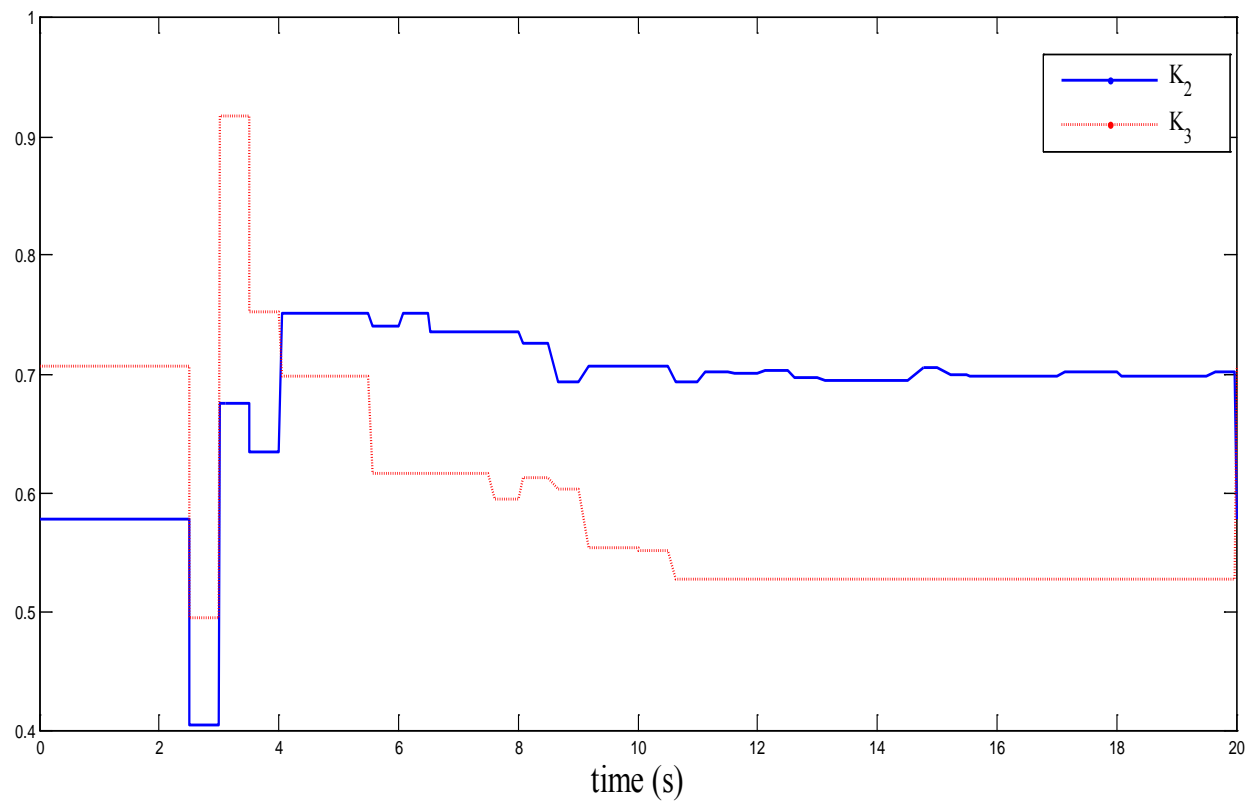

Figure5. 13: Operating Condition II -Scenario II parameters $K_{2}, K_{3}$

Results for both operating conditions substantiate that the reinforcement learning is suitable to be implemented in excitation control environment. In summary, the design of decentralized synergetic controllers with variable parameters is proposed in this chapter. Firstly, synergetic control theory is employed in designing nonlinear decentralized synergetic controllers. The parameters of the controllers are initialized using Particle Swarm Optimization. Reinforcement Learning is then employed to adjust or vary two of the five controller parameters. These two parameters, $\lambda_{i}, K_{i}$, are selected because their values have more effect on the efficiency of the controller: $K_{i}$ dictates the speed at which the system variables converge on the stable manifold, and $\lambda_{i}$ determines the motion of system afterward. The approach is demonstrated using a two area system. The results show that the proposed approach provides better performance than the fixed parameter synergetic controller, and power system stabilizer with fixed gains. 


\section{Chapter 6}

\section{SUMMARY AND CONCLUSIONS}

\subsection{Completed Work}

This dissertation proposes a synergetic control design to improve the transient stability of power system subject to contingencies. Each generator in the system is considered as a subsystem and a nonlinear decentralized synergetic controller is designed for it. Particle swarm Optimization technique is presented and utilized to obtain optimum values of the parameters of the designed controller. To achieve decentralized control, the coupling term between each subsystem and the rest of the system is considered as uncertainties. This uncertainty is estimated with a linear estimator whose coefficients are obtained adaptively. This uncertain function is expressed as a quadratic function of electric power, and its parameters are obtained by adaptation laws through adaptive synergetic technique.

Although the proposed synergetic controller enhances the dynamic performance greatly, the coordination of SVC supplementary controller and synergetic controller is even more promising. Lead-lag compensation is employed as the SVC supplementary controller. The problem of coordinating these two controllers is formulated as an optimization problem based on PSO so as to obtain the optimum gains of the SVC damping controller and the optimum values of the parameters of the synergetic controller. By selecting proper objective function during optimization of nonlinear controllers, great improvement on system performance is achieved.

The combination of reinforcement learning and synergetic controller is also proposed for generating units. The RL successively learns and adapts the synergetic controller' parameters to cover a wide range of operating conditions. 


\subsection{Suggestions for Future work}

The work in this dissertation focused on designing decentralized controller for generating units. Although the performances of nonlinear controllers are notable, their major drawback is the fact that they are mainly model based. This means unavailable information about the system may be required for their implementation. More focus should be made on techniques that are less depended on system model in designing excitation supplementary controllers.

\subsection{Publications}

1. Taoridi Ademoye, Ali Feliachi, "Decentralized Synergetic Control of Multimachine Systems" PSCE, IEEE Power Systems And Exposition, Phoenix, AZ, March 2011.

\section{Abstract:}

This paper proposes decentralized synergetic control approach to control a multimachine nonlinear electric power system through the excitation subsystem. The interconnection between generators is modeled as a function of the local variables and is considered as external disturbance to each subsystem. The nonlinear decentralized controllers effectively dampen the rotor angle oscillations and regulate the terminal voltages when a fault occurs. Two test systems are used to verify the efficiency of the proposed method against the conventional power system stabilizers: a nine- bus three- machine power system with inductive load, and the typical four machine two -area system.

2. T. Ademoye, A. Feliachi, and A. Karimi, Coordination of Synergetic Excitation Controller and SVC-Damping Controller Using Particle Swarm Optimization, in: IEEE Power and Energy Society General Meeting, Detroit, MI, July 24- 29,2011.

Abstract:

This paper addresses the enhancement of power system stability by simultaneous tuning of synergetic excitation damping controller and Static Var Compensator (SVC) - based damping controllers. Stable manifold is constructed for each excitation controller and based on that, an effective damping controller is derived. A lead -lag compensator is employed as a supplementary controller for the SVC. PSO algorithm is effectively utilized to simultaneously tune the parameters for the excitation damping controller(s) and the SVC 
supplementary controller. The coordination of the controllers effectively dampens the power angle oscillation and regulates the generator terminal voltage when a fault occurs.

3. T. Ademoye, A. Feliachi, Reinforcement Learning Tuned Decentralized Synergetic Control of Power Systems, Int. J Elsevier on Electric Power Systems Research 86 (2012) 34-40.

Abstract:

In this paper, decentralized synergetic controllers with varying parameters are developed to dampen oscillations in electric power systems via the excitation systems of the generators. Each generator is treated as a subsystem for which a synergetic controller is designed. Each subsystem is a dynamical system driven by a function that estimates the effect of the rest of the system. A Particle Swarm Optimization (PSO) technique is employed to initialize the controllers' gains. Then, Reinforcement Learning (RL) is used to vary the gains obtained after implementing the PSO so as to adapt the system to various operating conditions. Simulation results for a two area power system indicate that this technique gives a better performance than synergetic fixed gains controllers, or conventional power system stabilizers. 


\section{APPENDIX}

\section{A. Single Machine Infinite Bus System Data}

$\%$ Single-machine infinite bus example

$\%$ Base value

$\%$ Bus data format

$\%$ bus:

$\%$ col1 number

$\%$ col2 voltage magnitude (pu)

$\%$ col3 voltage angle (degree)

$\%$ col4 p-gen (pu)

$\%$ col5 q-gen (pu)

$\%$ col6 p-load (pu)

$\%$ col7 q-load (pu)

$\%$ col8 G-shunt (pu)

$\%$ col9 B-shunt (pu)

$\%$ col10 bus type

$\%$ bus type - 1 . swing bus

$\% \quad-2$. generator bus (PV bus)

$\% \quad-3$. load bus (PQ bus)

$\%$ col11 q-gen_max (pu)

$\%$ col12 q-gen_min (pu)

$\%$ col13 v_rated $(\mathrm{kV})$

$\%$ col14 v_max (pu)

$\%$ col15 v_min (pu)

bus $=[\ldots$

$\begin{array}{llllllllllll}1 & 1.00 & 0.00 & 0.9 & 0.436 & 0.00 & 0.00 & 0.00 & 0.00 & 2 & 99 & -99 ;\end{array}$

$\begin{array}{llllllllllll}2 & 1.00 & 0.00 & 0.00 & 0.00 & 0.00 & 0.00 & 0.00 & 0.00 & 3 & 99 & -99 ;\end{array}$

$\begin{array}{llllllllllll}3 & 1.00 & 0.00 & 0.00 & 0.00 & 00.0 & 0.00 & 0.00 & 0.00 & 1 & 99 & -99] ;\end{array}$

$\%$ Line data format: from bus, to bus, resistance $(\mathrm{pu})$, reactance $(\mathrm{pu})$,

$\% \quad$ line charging (pu), tap ratio, tap phase

line $=[\ldots$

$\begin{array}{lllllll}1 & 2 & 0.00 & 0.2 & 0.0 & 1 & 0.0 \text {; }\end{array}$

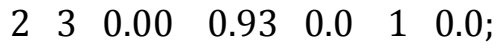

$\begin{array}{lllllll}2 & 3 & 0.00 & 0.5 & 0.0 & 1 & 0.0] \text {; }\end{array}$

$\%$ Machine data

$\%$ Machine data format:

$\%$ 1. machine number, 
$\%$ 2. bus number,

$\%$ 3. base mva,

$\%$ 4. leakage reactance $\mathrm{x}_{-} \mathrm{l}(\mathrm{pu})$

$\%$ 5. resistance $r_{-} a(p u)$

$\%$ 6. d-axis sychronous reactance $x_{-} d(p u)$

$\%$ 7. d-axis transient reactance $\mathrm{x}_{-}^{\prime} \mathrm{d}(\mathrm{pu})$

$\%$ 8. d-axis subtransient reactance $x^{\prime \prime} d(p u)$

$\%$ 9. d-axis open-circuit time constant T'_do(sec)

$\%$ 10. d-axis open-circuit subtransient time constant T"_do(sec)

$\%$ 11. q-axis sychronous reactance

$\%$ 12. q-axis transient reactance $\mathrm{x}_{-}^{\prime} \mathrm{q}(\mathrm{pu})$

$\%$ 13. q-axis subtransient reactance $x^{\prime \prime} \_q(p u)$

$\%$ 14. q-axis open-circuit time constant T'_qo(sec)

$\%$ 15. q-axis open circuit subtransient time constant T"_qo(sec)

$\%$ 16. inertia constant $\mathrm{H}(\mathrm{sec})$

$\%$ 17. damping coefficient d_o(pu)

$\%$ 18. dampling coefficient d_1(pu)

$\%$ 19. bus number

$\%$ 20. saturation factor $\mathrm{S}(1.0)$

$\%$ 21. saturation factor $\mathrm{S}(1.2)$

mac_con $=[\ldots$

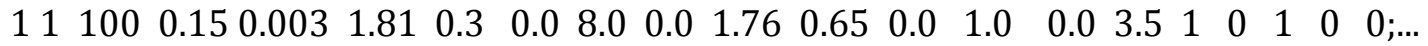

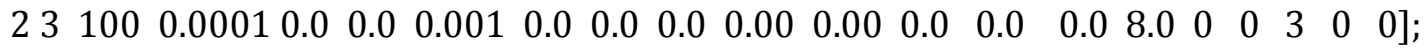

$\%$ Define infinite bus

ibus_con $=\left[\begin{array}{ll}0 & 1\end{array}\right]$;

\% Exciter data format:

$\%$ column data

$\% \quad 1$ - exciter type (1 for DC1, 2 for DC2)

$\% \quad 2$ - machine number

$\% 3$ - input filter time constant T_R

$\% \quad 4$ - voltage regulator gain K_A

$\% 5$ - voltage regulator time constant T_A

$\% 6$ - voltage regulator time constant T_B

$\% 7$ - voltage regulator time constant T_C

$\% 8$ - maximum voltage regulator output $V_{-}$Rmax

$\% 9$ - minimum voltage regulator output V_Rmin

$\% 10$ - exciter constant K_E

$\% 11$ - exciter time constant T_E

$\% 12$ - E_1

$\% 13$ - saturation function S_E(E_1)

$\% 14-$ E_2

$\% 15$ - saturation function S_E(E_2)

$\% 16$ - stabilizer gain K_F

$\% 17$ - stabilizer time constant $\mathrm{T}_{-} \mathrm{F}$

exc_con = $\left[\begin{array}{llllllllllllllll}0 & 1 & 0 & 200 & 0.015 & 0 & 0 & 15.0 & -15.0 & 0 & 0 & 0 & 0 & 0 & 0 & 0\end{array}\right]$;

$\%$ thermal turbine/governor model 
\%column data unit

$\% 1$ turbine model number $(=1)$

$\% 2$ machine number

$\% 3$ speed set point wf $\mathrm{pu}$

$\% 4$ steady state gain $1 / \mathrm{R}$ pu

$\% 5$ maximum power order Tmax pu on generator base

$\% 6$ servo time constant Ts sec

$\% 7$ governor time constant Tc sec

$\% 8$ transient gain time constant $\mathrm{T} 3 \mathrm{sec}$

$\% 9$ HP section time constant T4 sec

$\% 10$ reheater time constant T5 sec

tg_con = [ $\left.\begin{array}{llllllllll}1 & 1 & 1 & 25.0 & 17.5 & 0.1 & 0.5 & 0.0 & 1.25 & 5.0\end{array}\right]$;

$\%$ PSS data format:

$\%$ col1 type

$\%$ col2 gen number

$\%$ col3 gain (pu)

$\%$ col4 washout (sec)

$\%$ col5 lead T_1

$\%$ col6 lag T_2

$\%$ col7 lead T_3

$\%$ col8 lag T_4

$\%$ col9 V-max (pu)

$\%$ col10 V-min (pu)

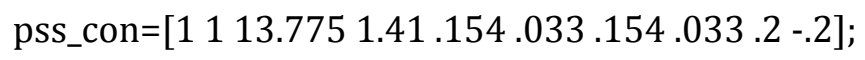

$\%$ dyn_load bus

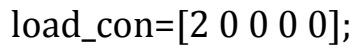

$\%$ SVC / STATCOM data format:

$\% 1$ - bus

$\% 2$ - reverence $|\mathrm{V}|$ at bus

$\% 3$ - Control flag: 0 - fixed reactance/ voltage

$\% \quad 1$ - voltage magnitude

$\% 4$ - model flag: $\quad 0$ - SVC

$\% \quad 1$ - STATCOM

$\% 5$ - transformer reactance [pu]

$\% 6-|\mathrm{Xc}|$

$\% 7-\mathrm{Xl} / \mathrm{Xc}$

$\% 8$ - LF solution

$\% 9$ - critical firing angle

$\% 10$ - dc side capacitor [pu]

$\% 11$ - dc side voltage level [kV]

$\% 12$ - ac base voltage $[\mathrm{kV}]$

$\% 13$ - transformer turn ratio

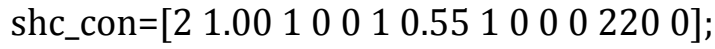


\%shc_con=[l2 1.00100010 .55000002200$]$;

\% The internal PI controller of the SVC

$\%$ SVCM Controller

$\% 1$ SVCM number

$\% 2-1=$ controller active (on), $0=$ controller inactive (off)

$\% 3$ - lq_ref P-gain

$\% 4$ - lq_ref I-gain

$\% 5-$

$\% 6-$

$\% 7$ - limit abs(svcm_I) in pu

$\% 8$ -

svcm_ctrl = $\left[\begin{array}{llllllll}1 & 1 & 20 & 0.3 & 0 & 0 & 5 & 0\end{array}\right] ;$ 


\section{B. Three Machine Nine Bus System(with Induction Motor) Data}

\% System base MVA setting (if none default of 100 MVA is taken)

basmva=100;

$\%$ bus data format

$\%$ bus:

$\%$ col1 number

$\%$ col2 voltage magnitude(pu)

$\%$ col3 voltage angle(degree)

$\%$ col4 p_gen(pu)

$\%$ col5 q_gen(pu),

$\%$ col6 p_load(pu)

$\%$ col7 q_load(pu)

$\%$ col8 G shunt(pu)

$\%$ col9 B shunt(pu)

$\%$ col10 bus_type

$\%$ bus_type - 1 , swing bus

$\% \quad-2$, generator bus (PV bus)

$\% \quad-3$, load bus (PQ bus)

$\%$ col11 q_gen_max(pu)

$\%$ col12 q_gen_min(pu)

$\%$ col13 v_rated $(\mathrm{kV})$

$\%$ col14 v_max pu

$\%$ col15 v_min pu

bus $=[\ldots$

$\begin{array}{lllllllllllllll}1 & 1.04 & 0 & 0.716 & 0.27 & 0.0 & 0.0 & 0.0 & 0.0 & 1 & 20 & -20 & 138 & 1.1 & 0.9 \text {; }\end{array}$

$\begin{array}{lllllllllllllll}2 & 1.025 & 0 & 1.63 & 0.067 & 0.0 & 0.0 & 0.0 & 0.0 & 2 & 20 & -20 & 138 & 1.1 & 0.9 \text {; }\end{array}$

$\begin{array}{lllllllllllllll}3 & 1.025 & 0 & 0.85 & -0.0109 & 0.0 & 0.0 & 0.0 & 0.0 & 2 & 20 & -20 & 138 & 1.1 & 0.9 \text {; }\end{array}$

$\begin{array}{lllllllllllllll}4 & 1.0 & 0 & 0.0 & 0.0 & 0.0 & 0.0 & 0.0 & 0.0 & 3 & 20 & -20 & 138 & 1.1 & 0.9 \text {; }\end{array}$

$\begin{array}{lllllllllllllll}5 & 1.0 & 0 & 0.0 & 0.0 & 1.25 & 0.5 & 0.0 & 0.0 & 3 & 20 & -20 & 138 & 1.1 & 0.9 \text {; }\end{array}$

$\begin{array}{lllllllllllllll}6 & 1.0 & 0 & 0.0 & 0.0 & 0.9 & 0.3 & 0.0 & 0.0 & 3 & 20 & -20 & 138 & 1.1 & 0.9 \text {; }\end{array}$

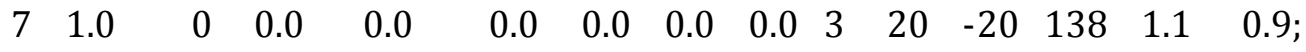

$\begin{array}{lllllllllllllll}8 & 1.0 & 0 & 0.0 & 0.0 & 1.0 & 0.35 & 0.0 & 0.0 & 3 & 20 & -20 & 138 & 1.1 & 0.9 \text {; }\end{array}$

$\begin{array}{lllllllllllllll}9 & 1.0 & 0 & 0.0 & 0.0 & 0.0 & 0.0 & 0.0 & 0.0 & 3 & 20 & -20 & 138 & 1.1 & 0.9] ;\end{array}$

$\%$ line data format

$\%$ line: col 1-from bus,

$\%$ col 2-to bus,

$\%$ col 3-resistance(pu),

$\%$ col 4-reactance(pu),

$\%$ col 5-line charging(pu),

$\%$ col 6-tap ratio,

$\%$ col 7-tap phase,

$\%$ col 8-tapmax,

$\%$ col 9-tapmin,

$\%$ col 10-tap size 


\begin{tabular}{llllllllll}
\multicolumn{2}{l}{ line $=[\cdots$} \\
1 & 4 & 0.0 & 0.0567 & 0.0 & 0.0 & 0.0 & 0.0 & 0.0 & $0.00 ;$ \\
2 & 7 & 0.0 & 0.0625 & 0.0 & 0.0 & 0.0 & 0.0 & 0.0 & $0.00 ;$ \\
3 & 9 & 0.0 & 0.0586 & 0.0 & 0.0 & 0.0 & 0.0 & 0.0 & $0.00 ;$ \\
4 & 5 & 0.01 & 0.085 & $0.088^{*} 2$ & 0.0 & 0.0 & 0.0 & 0.0 & $0.00 ;$ \\
4 & 6 & 0.017 & 0.092 & $0.079 * 2$ & 0.0 & 0.0 & 0.0 & 0.0 & $0.00 ;$ \\
5 & 7 & 0.032 & 0.161 & $0.153^{*} 2$ & 0.0 & 0.0 & 0.0 & 0.0 & $0.00 ;$ \\
6 & 9 & 0.039 & 0.170 & $0.179 * 2$ & 0.0 & 0.0 & 0.0 & 0.0 & $0.00 ;$ \\
7 & 8 & 0.0085 & 0.072 & $0.0745^{*} 2$ & 0.0 & 0.0 & 0.0 & 0.0 & $0.00 ;$ \\
8 & 9 & 0.0119 & 0.1008 & $0.1045^{*} 2$ & 0.0 & 0.0 & 0.0 & 0.0 & $0.00] ;$
\end{tabular}

$\%$ Machine data format

$\%$ 1. machine number,

$\%$ 2. bus number,

$\% \quad 3$. base mva,

$\%$ 4. leakage reactance $\mathrm{x}_{-} \mathrm{l}(\mathrm{pu})$,

$\%$ 5. resistance $r_{-} a(p u)$,

$\% \quad$ 6. d-axis sychronous reactance $\mathrm{x}_{-} \mathrm{d}(\mathrm{pu})$,

$\% \quad$ 7. d-axis transient reactance $x^{\prime} \_d(p u)$,

$\%$ 8. $d$-axis subtransient reactance $x^{\prime \prime} d(p u)$,

$\%$ 9. d-axis open-circuit time constant T'_do(sec),

$\%$ 10. d-axis open-circuit subtransient time constant

$\% \quad$ T"_do(sec),

$\% \quad$ 11. q-axis sychronous reactance $x_{-} q(\mathrm{pu})$,

$\% \quad$ 12. $q$-axis transient reactance $\mathrm{x}_{-}^{\prime} \mathrm{q}(\mathrm{pu})$,

$\% \quad$ 13. q-axis subtransient reactance $x^{\prime \prime} \_q(p u)$,

$\% \quad$ 14. q-axis open-circuit time constant T"_qo(sec),

$\% \quad 15$. q-axis open circuit subtransient time constant

$\% \quad$ T"_qo(sec),

$\% \quad$ 16. inertia constant $\mathrm{H}(\mathrm{sec})$,

$\% \quad$ 17. damping coefficient d_o(pu),

$\%$ 18. dampling coefficient d__1(pu),

$\% \quad$ 19. bus number

\%transient model

mac_con $=[\ldots$

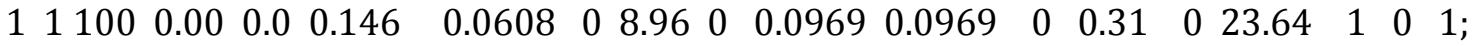

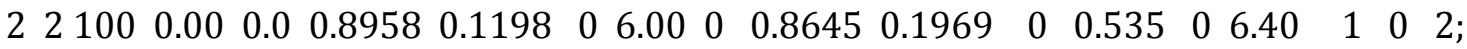

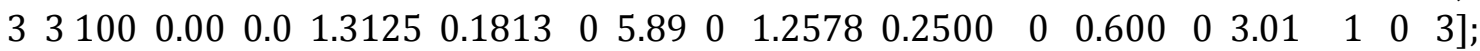

$\%$ Exciter Model format

$\% \quad$ 1. type (1=DC1, 2=DC2, 3=st3)

$\% \quad$ 2. machine \#

$\% \quad 3$. input filter time constant, $\operatorname{Tr}$ (sec)

$\%$ 4. voltage regulator gain, $\mathrm{Ka}$

$\% \quad 5$. voltage regulator time constant, Ta (sec)

$\% \quad 6$. voltage regulator time constant, $\mathrm{Tb}(\mathrm{sec})$ 


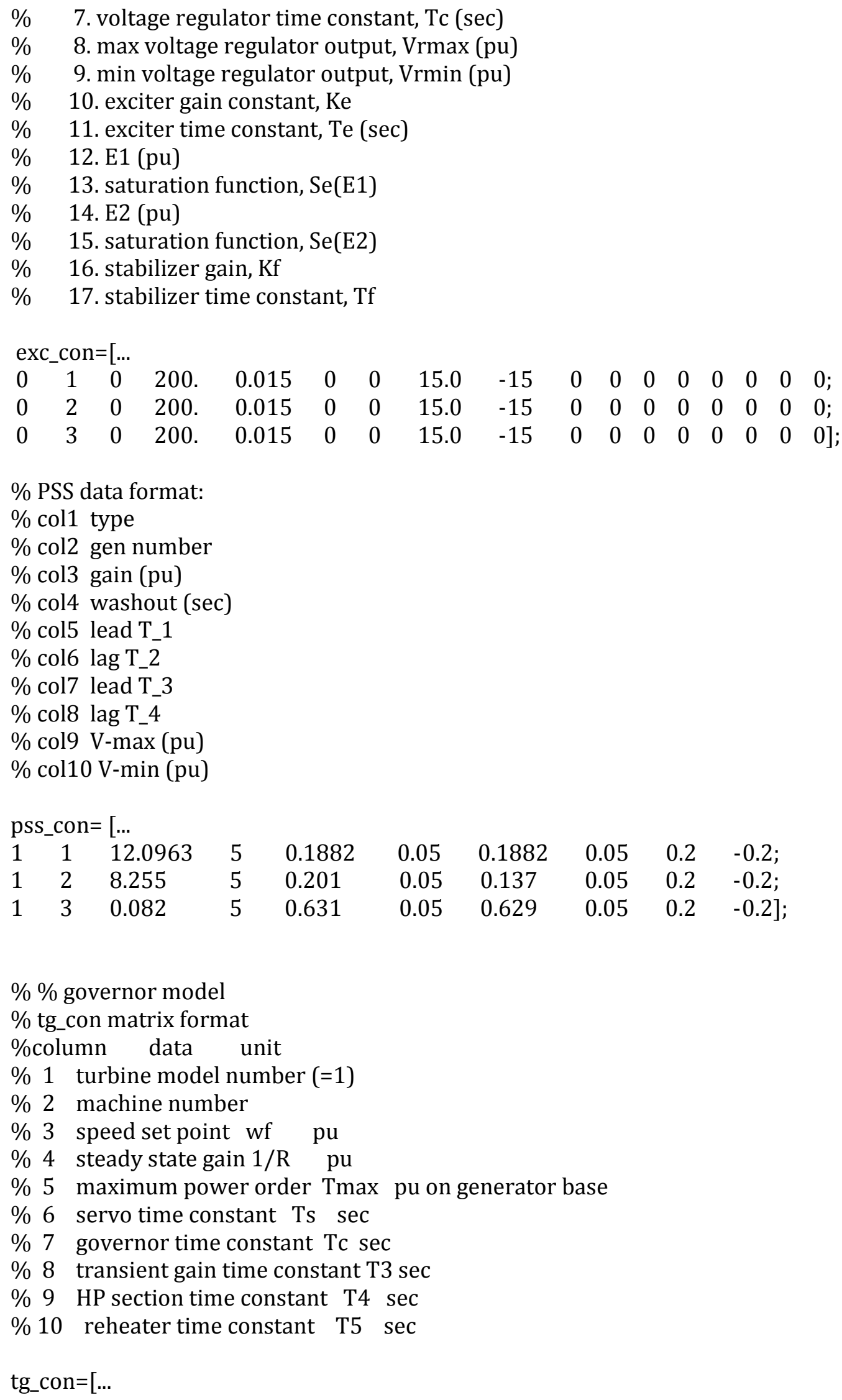




$\begin{array}{llllllllll}1 & 1 & 1 & 25.0 & 2.0 & 0.1 & 0.5 & 0.0 & 1.25 & 5.0 ; \\ 1 & 2 & 1 & 25.0 & 2.0 & 0.1 & 0.5 & 0.0 & 1.25 & 5.0 ; \\ 1 & 3 & 1 & 25.0 & 2.0 & 0.1 & 0.5 & 0.0 & 1.25 & 5.0] ;\end{array}$

$\%$ Load configuration

$\% 1$ bus number

$\% 2$ fracation of constant $\mathrm{P}$

$\% 3$ fracation of constant $\mathrm{Q}$

$\% 4$ fracation of constant active current

$\% 5$ fracation of constant reactive current

$\%$ Simple Induction Motor Model - Single Cage, no leakage inductance saturation

$\%$ ind_con $=[\ldots$

$\% 1$ - motor number

$\% 2$ - busnumber

$\% 3$ - base MVA

$\% 4-r s$

$\% 5$ - xs -stator leakage reactance

$\% 6$ - Xm - magnetizing reactance

$\% 7-\mathrm{rr}$

$\% 8$ - xr - rotor leakage reactance

$\% 9-\mathrm{H}$ - inertia constant motor + load in sec

$\%$ 10-14 Don't care (Not Apllicable)

$\% 15$ - fraction of bus load power taken by motor

$\%$ Induction Motor Load Torque Characteristic (function of slip)

$\%$

$\%$ mld_con $=[\ldots$

$\%$

$\% 1$ motor number

$\% 2$ bus number

$\% 3$ stiction load pu on motor base (f1)

$\% 4$ stiction load coefficient

$\% 5$ external load pu on motor base (f2)

$\% 6$ external load coefficient (i2)

$\%$

$\%$ load has the form: torque $=\mathrm{f} 1{ }^{*} \operatorname{slip}^{\wedge} \mathrm{i} 1+\mathrm{f} 2^{*}(1-\text { slip })^{\wedge} \mathrm{i} 2$

$\%$ per-unit system for induction generator

rated_voltage $=660 ; \%[\mathrm{~V}]$

rated_power $=250 ; \%[\mathrm{~kW}]$

base_Z = rated_voltage ${ }^{\wedge} 2$ / (rated_power*1000);

$\%$ Induction Generator data format

ind_con $=[\ldots$

$180.25 \quad .00708 /$ base_Z $\quad .0762 /$ base_Z $\quad 3.44979 /$ base_Z $\quad .00759 /$ base_Z ... $.23289 /$ base_Z $\quad 8.22 * 1 \quad 0 \quad 000006]$;

mld_con $=\left[\begin{array}{llllll}1 & 8 & 2 & 1 & 2 & 1.5\end{array}\right]$; 


\title{
C. Two Area-Four Machine System Data
}

\author{
$\%$ Two Area Four Machine Test Case
}

$\%$

$\%$ This is one of the most famous power system examples EVER! More information

$\%$ can be found in either of the following books:

\% Prabha Kundur, Power System Stability and Control, McGraw-Hill Inc., 1994

$\%$ Graham Rogers, Power System Oscillations, M. A. Pai, Editor, Norwell: Kluwer Academic

Publishers, 2000

$\%$ bus data

bus $=[\ldots$

$\begin{array}{lcccccccccccccl}1 & 1.03 & 0 & 7.00 & 1.85 & 0 & 0 & 0 & 0 & 1 & 99.0 & -99.0 & 22.0 & 1.1 & .9 ; \\ 2 & 1.01 & 0 & 7.00 & 2.35 & 0 & 0 & 0 & 0 & 2 & 99.0 & -99.0 & 22.0 & 1.1 & .9 ; \\ 3 & 0.9781 & 0 & 0.00 & 2.00 & 0 & 0 & 0 & 0 & 3 & 0.0 & 0.0 & 500.0 & 1.5 & .5 ; \\ 4 & 0.95 & 0 & 0.00 & 0.00 & 9.67 & 1 & 0 & 0 & 3 & 0.0 & 0.0 & 115.0 & 1.05 & .95 ; \\ 10 & 1.0103 & 0 & 0.00 & 0.00 & 0 & 0 & 0 & 0 & 3 & 0.0 & 0.0 & 230.0 & 1.5 & .5 ; \\ 11 & 1.03 & 0 & 7.19 & 1.76 & 0 & 0 & 0 & 0 & 2 & 99.0 & -99.0 & 22.0 & 1.1 & .9 ; \\ 12 & 1.01 & 0 & 7.00 & 2.02 & 0 & 0 & 0 & 0 & 2 & 99.0 & -99.0 & 22.0 & 1.1 & .9 ; \\ 13 & 0.9899 & 0 & 0.00 & 3.50 & 0 & 0 & 0 & 0 & 3 & 0.0 & 0.0 & 500.0 & 1.5 & .5 ; \\ 14 & 0.95 & 0 & 0.00 & 0.00 & 17.67 & 1 & 0 & 0 & 3 & 0.0 & 0.0 & 115.0 & 1.05 & .95 ; \\ 20 & 0.9876 & 0 & 0.00 & 0.00 & 0 & 0 & 0 & 0 & 3 & 0.0 & 0.0 & 230.0 & 1.5 & .5 ; \\ 101 & 1.0 & 0 & 0.00 & 0.00 & 0 & 0 & 0 & 1.60 & 3 & 99.0 & -99.0 & 500.0 & 1.5 & .5 ; \\ 110 & 1.0125 & 0 & 0.00 & 0.00 & 0 & 0 & 0 & 0 & 3 & 0.0 & 0.0 & 230.0 & 1.5 & .5 ; \\ 120 & 0.9938 & 0 & 0.00 & 0.00 & 0 & 0 & 0 & 0 & 3 & 0.0 & 0.0 & 230.0 & 1.5 & .5] ;\end{array}$

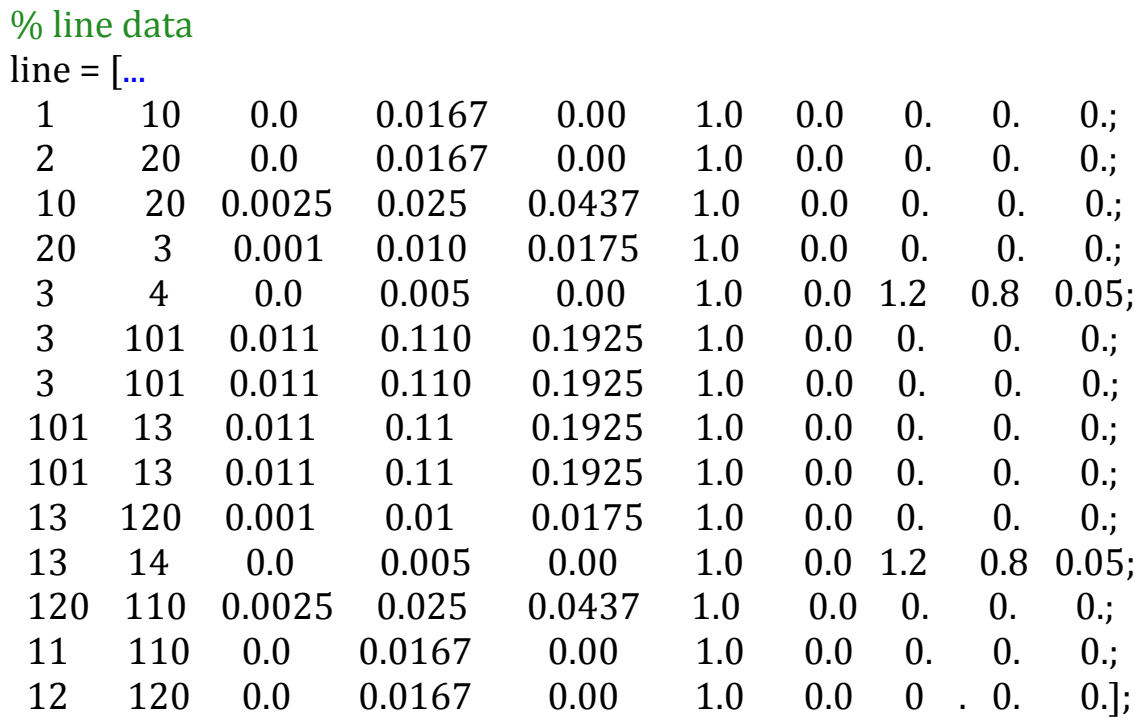


$\%$ mac_con $=[\ldots$

$\%$ note: all the following machines use sub-transient model

$\% \quad 1$. machine number,

$\%$ 2. bus number,

$\% \quad 3$. base mva,

$\%$ 4. leakage reactance $\mathrm{x}_{-} \mathrm{l}(\mathrm{pu})$

$\% \quad$ 5. resistance $r_{-} a(\mathrm{pu})$

$\% \quad 6 . d$-axis sychronous reactance $x_{-} d(p u)$

$\% \quad$ 7. d-axis transient reactance $x_{-}^{\prime} d(p u)$

$\%$ 8. d-axis subtransient reactance $x^{\prime \prime} d(p u)$

$\%$ 9. d-axis open-circuit time constant T'_do(sec)

$\%$ 10. d-axis open-circuit subtransient time constant T"_do(sec)

$\% \quad$ 11. q-axis sychronous reactance $\mathrm{x} \_\mathrm{q}(\mathrm{pu})$

$\% \quad$ 12. q-axis transient reactance $\mathrm{x}_{-}^{\prime} \mathrm{q}(\mathrm{pu})$

$\%$ 13. q-axis subtransient reactance $x^{\prime \prime}+q(p u)$

$\% \quad$ 14. q-axis open-circuit time constant T'_qo(sec)

$\% \quad$ 15. q-axis open circuit subtransient time constant T"_qo(sec)

$\% \quad$ 16. inertia constant $\mathrm{H}(\mathrm{sec})$

$\% \quad$ 17. damping coefficient d_o(pu)

$\%$ 18. dampling coefficient d_1(pu)

$\% \quad 19$. bus number

\%Transient Model

mac_con $=[\ldots$

$\begin{array}{lllllllllllllllllll}1 & 1 & 900 & 0.200 & 0.0025 & 1.8 & 0.30 & 0.00 & 8.00 & 0.00 & 1.7 & 0.55 & 0.00 & 0.4 & 0.00 & 6.5 & 1 & 0 & 1 \text {; }\end{array}$

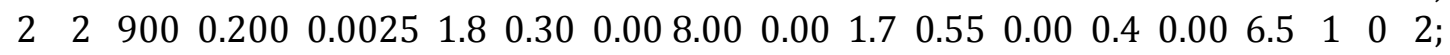

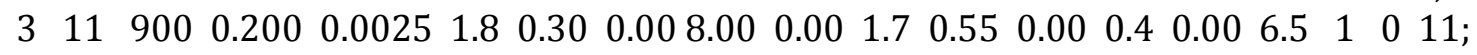

$\begin{array}{lllllllllllllllllll}4 & 12 & 900 & 0.200 & 0.0025 & 1.8 & 0.30 & 0.00 & 8.00 & 0.00 & 1.7 & 0.55 & 0.00 & 0.4 & 0.00 & 6.5 & 1 & 0 & 12] ;\end{array}$

$\%$ Exciter data

exc_con $=[\cdots$

$\%$ Simple exciter

$\% \quad$ filter regulator regulator lag lead max min

$\% \quad$ gen time $\mathrm{Tr}$ gain $\mathrm{Ka}$ time Ta time $\mathrm{Tb}$ time Tc $\mathrm{Vr} \mathrm{Vr}$

$\%$ type no. (sec.) (pu) (sec.) (sec.) (sec.) (pu) (pu)

$\begin{array}{llllllllllllllllllll}0 & 1 & 0.01 & 200 & 0 & 0 & 0 & 10 & -10 & 0 & 0 & 0 & 0 & 0 & 0 & 0 & 0 & 0 & 0 & 0 \text {; }\end{array}$

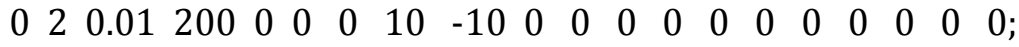

$\begin{array}{llllllllllllllllllll}0 & 3 & 0.01 & 200 & 0 & 0 & 0 & 10 & -10 & 0 & 0 & 0 & 0 & 0 & 0 & 0 & 0 & 0 & 0 & 0 ;\end{array}$

$\left.\begin{array}{llllllllllllllllllll}0 & 4 & 0.01 & 200 & 0 & 0 & 0 & 10 & -10 & 0 & 0 & 0 & 0 & 0 & 0 & 0 & 0 & 0 & 0 & 0\end{array}\right]$;

$\%$ Turbine-governor data

$\%$ column data unit

$\% \quad 1$ turbine model number $(=1)$

$\% 2$ machine number

$\% 3$ speed set point $\quad$ wf pu

$\% 4$ steady state gain $1 / \mathrm{R} \mathrm{pu}$

\% 5 maximum power order Tmax pu on generator base

$\% 6$ servo time constant Ts sec

$\% 7$ governor time constant Tc sec 
\% 8 transient gain time constant T3 sec

$\% 9$ HP section time constant T4 sec

$\% 10$ reheater time constant T5 sec

tg_con $=[\ldots$

$\% \begin{array}{lllllllllll}\% & 1 & 2 & 3 & 4 & 5 & 6 & 7 & 8 & 9 & 10\end{array}$

$\begin{array}{llllllllll}1 & 1 & 1 & 25.0 & 1.0 & 0.1 & 0.5 & 0.0 & 1.25 & 5.0 \text {; }\end{array}$

$\begin{array}{lllllllllll}1 & 2 & 1 & 25.0 & 1.0 & 0.1 & 0.5 & 0.0 & 1.25 & 5.0 \text {; }\end{array}$

$\begin{array}{lllllllllll}1 & 3 & 1 & 25.0 & 1.0 & 0.1 & 0.5 & 0.0 & 1.25 & 5.0 \text {; }\end{array}$

$\begin{array}{lllllllllll}1 & 4 & 1 & 25.0 & 1.0 & 0.1 & 0.5 & 0.0 & 1.25 & 5.0]\end{array}$

pss_con $=[\ldots$

$1247.1620 .00 .71090 .1550 .71090 .1550 .2-0.05$;

1330020.00 .150 .084310 .150 .084310 .2 -0.05]; 


\section{Fifty Machine System Data}

\% 50 Machine System Data:

$\%$ This is a 50-machine system with 145 buses from Iowa State University.

$\%$ The data has been modified to include full generator and control data

$\%$ Some em generator bus data has been modified to stabilize unrealistic instabilities

$\%$ Machine data format

$\% \quad 1$ machine number,

$\% \quad 2$ bus number,

$\% 3$ base mva,

$\% \quad 4$ leakage reactance $\mathrm{x}_{-} 1(\mathrm{pu})$,

$\% 5$ resistance $r_{-} \mathrm{a}(\mathrm{pu})$,

$\% 6 \mathrm{~d}$-axis sychronous reactance $\mathrm{x}_{-} \mathrm{d}(\mathrm{pu})$,

$\% \quad 7 \mathrm{~d}$-axis transient reactance $\mathrm{x}^{\prime} \mathrm{d}(\mathrm{p}(\mathrm{pu})$,

$\% \quad 8 \mathrm{~d}$-axis subtransient reactance $\mathrm{x}_{\text {"_d }} \mathrm{d}(\mathrm{pu})$,

$\% 9 \mathrm{~d}$-axis open-circuit time constant T'_do(sec),

$\% 10 \mathrm{~d}$-axis open-circuit subtransient time constant

$\% \quad$ T"_do(sec),

$\% \quad 11$ q-axis sychronous reactance $x_{-} q(\mathrm{pu})$,

$\% \quad 12$ q-axis transient reactance $x_{-}^{\prime} q(p u)$,

$\% 13$ q-axis subtransient reactance $x^{\prime \prime} q(p u)$,

$\% 14$ q-axis open-circuit time constant T'_qo(sec),

$\% \quad 15$ q-axis open circuit subtransient time constant

$\% \quad$ T"_qo(sec),

$\% \quad 16$ inertia constant $\mathrm{H}(\mathrm{sec})$,

$\% 17$ damping coefficient d_o(pu),

$\% 18$ dampling coefficient d_1(pu),

$\% 19$ bus number

$\% \quad 20$ saturation factor $\mathrm{s}(1.0)$

$\% \quad 21$ saturation factor $\mathrm{s}(1.2)$

$\% \quad 22$ active power fraction

$\% \quad 23$ reactive power fraction

mac_con $=[\ldots$

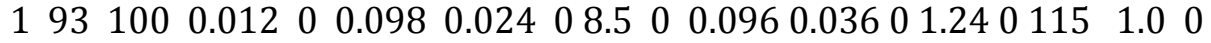
$930.06540 .5743 \quad 00$;

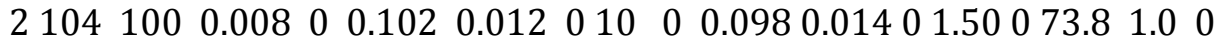

$1040.210 .55 \quad 00$;

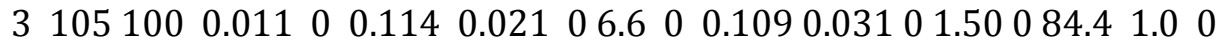

$1050.130 .4096 \quad 00$;

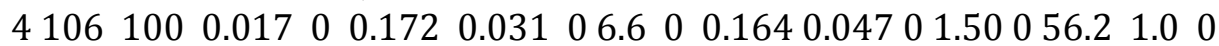

1060.130 .409600 ;

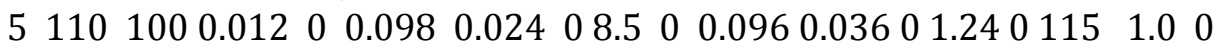

$\begin{array}{llll}110 & 0.0654 & 0.5743 & 0.0 \text {; }\end{array}$

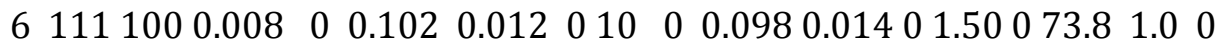

$\begin{array}{lllll}111 & 0.21 & 0.55 & 0 & 0\end{array}$

$\begin{array}{lllllllllllllllll}7 & 60 & 100 & 0.00 & 0.000 & 0 . & 0.4769 & 0 & 0 & 0 & 0 & 0 & 0 & 0 & 0 & 1.41 & 1.41\end{array}$ 
0.06000000 ;

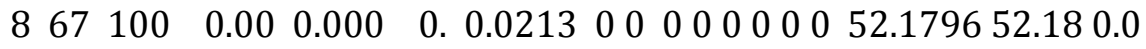

670000

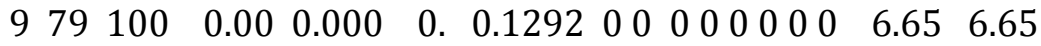

0.0790000 ;

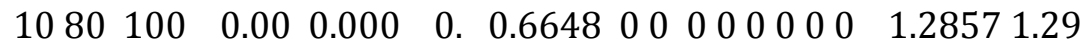

0.0800000 ;

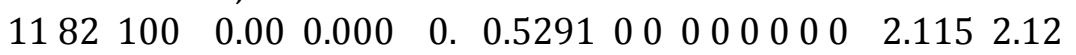

0.0820000 ;

$1289100 \quad 0.000 .000 \quad 0.0 .0585000000000020 .560220 .560 .0$

890000 ;

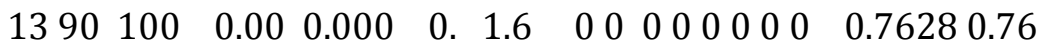

0.0900000 ;

$1491100 \quad 0.000 .000 \quad 0.0 .3718000000000001 .68481 .68$

0.0910000 ;

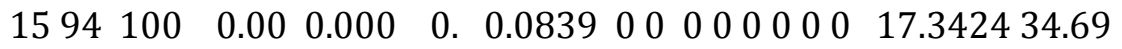

0.0940000 ;

$1695100 \quad 0.00 \quad 0.000 \quad 0 . \quad 0.161900000000000 \quad 5.46625 .46$

0.0950000 ;

$1796100 \quad 0.00 \quad 0.000 \quad 0 . \quad 0.4824000000000002 .12162 .12$

0.0960000 ;

$1897100 \quad 0.000 .000 \quad 0 . \quad 0.212500000000000 \quad 5.49125 .49$

0.0970000 ;

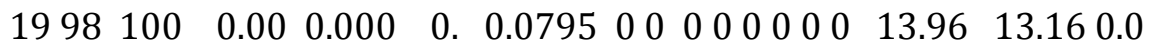

980000 ;

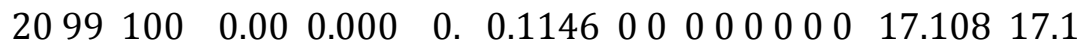

0.0990000 ;

$\begin{array}{lllllllllllllllll}21 & 100100 & 0.00 & 0.000 & 0 . & 0.1386 & 0 & 0 & 0 & 0 & 0 & 0 & 0 & 0 & 7.56 & 7.56\end{array}$

0.01000000 ;

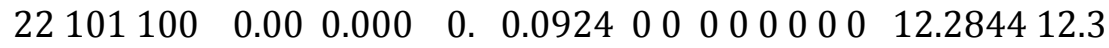

0.01010000 ;

$\begin{array}{llllllllllllllll}23102 & 100 & 0.00 & 0.000 & 0 . & 0.0135 & 0 & 0 & 0 & 0 & 0 & 0 & 0 & 0 & 78.43 & 78.4\end{array}$

0.01020000 ;

$24103100 \quad 0.000 .000 \quad 0.0 .106300000000008 .168 .16$

0.01030000 ;

$25108100 \quad 0.00 \quad 0.000 \quad 0.0 .02480000000000030 .43230 .4$

0.01080000 ;

$26109100 \quad 0.00 \quad 0.000 \quad 0.0 .2029000000000002 .66222 .66$

0.01090000 ;

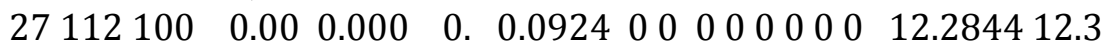

0.01120000 ;

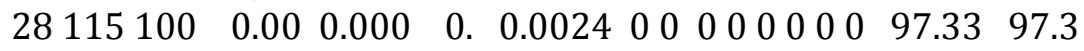

0.01150000 ;

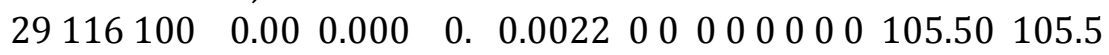

0.01160000 ;

$\begin{array}{lllllllllllllll}30117100 & 0.00 & 0.000 & 0 . & 0.0017 & 0 & 0 & 0 & 0 & 0 & 0 & 0 & 0 & 102.16 & 102.2\end{array}$

0.01170000 ;

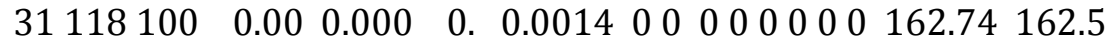

0.01180000 ;

$32119100 \quad 0.00 \quad 0.000 \quad 0 . \quad 0.00020000000000348 .22348 .2$ 
0.01190000 ;

$33121100 \quad 0.00 \quad 0.000 \quad 0.0 .00170000000000116 .54116 .5$

0.01210000 ;

$\begin{array}{llllllllllllllll}34 & 122 & 100 & 0.00 & 0.000 & 0.00089 & 0 & 0 & 0 & 0 & 0 & 0 & 0 & 0 & 39.24 & 39.2\end{array}$

0.01220000 ;

$\begin{array}{lllllllllllllll}35124100 & 0.00 & 0.000 & 0 . & 0.0017 & 0 & 0 & 0 & 0 & 0 & 0 & 0 & 0 & 116.86 & 116.9\end{array}$

0.01240000 ;

$36128100 \quad 0.00 \quad 0.000 \quad 0.0 .00010000000000503 .87503 .9$

0.01280000 ;

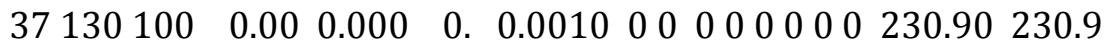

0.01300000 ;

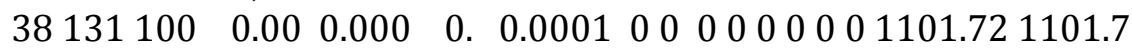

0.01310000 ;

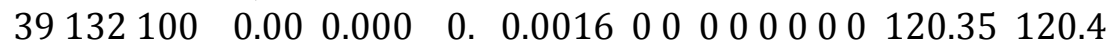

0.01320000 ;

$40134100 \quad 0.00 \quad 0.000 \quad 0 . \quad 0.3 e-4000000000802.12802 .1$

0.01340000 ;

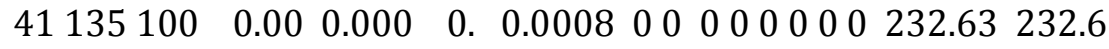

0.01350000 ;

$42136100 \quad 0.00 \quad 0.000 \quad 0 . \quad 0.1 \mathrm{e}-4 \quad 00000000002018.175000 .2$

0.01360000 ;

$43137100 \quad 0.00 \quad 0.000 \quad 0 . \quad 0.00040000000000469 .32469 .3$

0.01370000 ;

$44139100 \quad 0.00 \quad 0.000 \quad 0.0 .00010000000002210 .202210 .2$

0.01390000 ;

$45140100 \quad 0.00 \quad 0.000 \quad 0.0 .00030000000000889 .19889 .2$

0.01400000 ;

$46141100 \quad 0.000 .000 \quad 0.0 .000100000000001474 .221474 .2$

0.01410000 ;

$47142100 \quad 0.00 \quad 0.000 \quad 0.0 .00030000000000950 .80950 .8$

0.01420000 ;

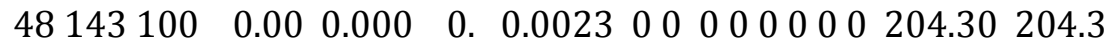

0.01430000 ;

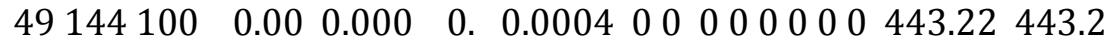

0.01440000 ;

$50145100 \quad 0.00 \quad 0.000 \quad 0.0 .00180000000000518 .08518 .1$

$0.01450000]$;

$\%$ Exciter data format

$\%$ column data

$\% 1$ exciter type

$\% 2$ machine number

$\% 3$ input filter time constant

$\% 4$ voltage regulator gain K_A

$\% 5$ voltage regulator time constant T_A (sec)

$\% 6$ voltage regulator time constant T_B (sec)

$\% 7$ voltage regulator time constant T_C (sec)

\% 8 maximum voltage regulator output VR_max

$\% 9$ minimum voltage regulator output VR_min

\% 10 exciter constant K_e 
$\% 11$ exciter time constant T_E

$\% 12$ E_1

$\% 13$ saturation function S_E (E_1)

$\% 14 \quad$ E_2

$\% 15$ saturation function S_E (E_2)

$\% 16$ stabilizer gain $\mathrm{K} \_\mathrm{f}$

$\% 17$ stabilizer time constant (T_f)

exc_con $=[\ldots$

$\begin{array}{lllllllllllllllll}0 & 1 & 0.02 & 185.0 & 0 & 0 & 0 & 8.89 & -2.0 & 0 & 0 & 0 & 0 & 0 & 0 & 0 & 0 \text {; }\end{array}$

$\begin{array}{lllllllllllllllll}0 & 2 & 0.015 & 253.0 & 0 & 0 & 0 & 8.86 & -7.0 & 0 & 0 & 0 & 0 & 0 & 0 & 0 & 0 \text {; }\end{array}$

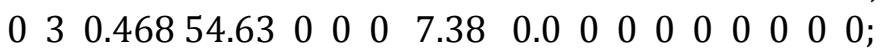

$\begin{array}{lllllllllllllllll}0 & 4 & 0.468 & 54.63 & 0 & 0 & 0 & 7.38 & 0.0 & 0 & 0 & 0 & 0 & 0 & 0 & 0 & 0 \text {; }\end{array}$

$\begin{array}{lllllllllllllllll}0 & 5 & 0.02 & 185.0 & 0 & 0 & 0 & 8.89 & -2.0 & 0 & 0 & 0 & 0 & 0 & 0 & 0 & 0 \text {; }\end{array}$

$\left.\begin{array}{lllllllllllllllll}0 & 6 & 0.015 & 253.0 & 0 & 0 & 0 & 8.86 & -7.0 & 0 & 0 & 0 & 0 & 0 & 0 & 0 & 0\end{array}\right]$; 


\title{
E. Matlab Codes
}

\section{Particle Swarm Optimization}

\author{
function [Jss,Xss,Jsss,t] = psomax(bounds,evalFN,tmax,c1,c2,n,M)
}

\% PSOmax run a Partical Swarm Optimization to maximize the objective function.

$\%$ function [x,endPop,bPop,traceInfo]=ga(bounds,evalFN,tmax,c1,c2,n,M)

$\%$

$\%$ Output Arguments:

$\%$ Jss - the best solution found during the course of the run.

$\%$ xss - the final population.

$\%$ Jsss - a trace of the best population.

$\% \mathrm{t} \quad$ - number of iteration.

$\%$

$\%$ Input Arguments:

$\%$ bounds - a matrix of upper and lower bounds on the variables.

$\%$ evalFN - the name of the evaluation .m function.

$\%$ tmax - maximum number of iteration.

$\%$ c1 - weighting factor.

$\%$ c2 - weighting factor.

$\% \mathrm{n} \quad$ - number of popualtion.

$\% \quad \mathrm{M} \quad-$ maximum number of iteration of unchanged soltion.

\% Partical Swarm Optimization for Matlab

$\%$ Copyright (C) 2003 Amer Alhinai

$\%$

$\%$ This program is free software; you can redistribute it and/or modify

$\%$ it under the terms of the GNU General Public License as published by

$\%$ the Free Software Foundation; either version 1, or (at your option)

$\%$ any later version.

$\%$

$\%$ This program is distributed in the hope that it will be useful,

$\%$ but WITHOUT ANY WARRANTY; without even the implied warranty of

$\%$ MERCHANTABILITY or FITNESS FOR A PARTICULAR PURPOSE. See the

$\%$ GNU General Public License for more details. A copy of the GNU

$\%$ General Public License can be obtained from the

\% Free Software Foundation, Inc., 675 Mass Ave, Cambridge, MA 02139, USA.

\%\%\%\%\%\%\%\%\%\%\%\%\%\%\%\%\%\%\%\%\%\%\%\%\%

x_int= bounds;

$\mathrm{t}=1 ; \quad \%$ 1st iteration.

$\%$ wmax $=0.9$;

$\%$ wmin $=0.4$;

$\% \mathrm{w}(\mathrm{t})=\mathrm{wmax}-((\mathrm{wmax}-\mathrm{wmin}) / \mathrm{tmax}) * \mathrm{t}$;

$\%$ r1 $=$ rand(tmax);r2=rand(tmax);

\%\%\%\%\%\%\%\%\%\%\%\%\%\%\%\%\%\%\%\%\%\%\%\%\% 


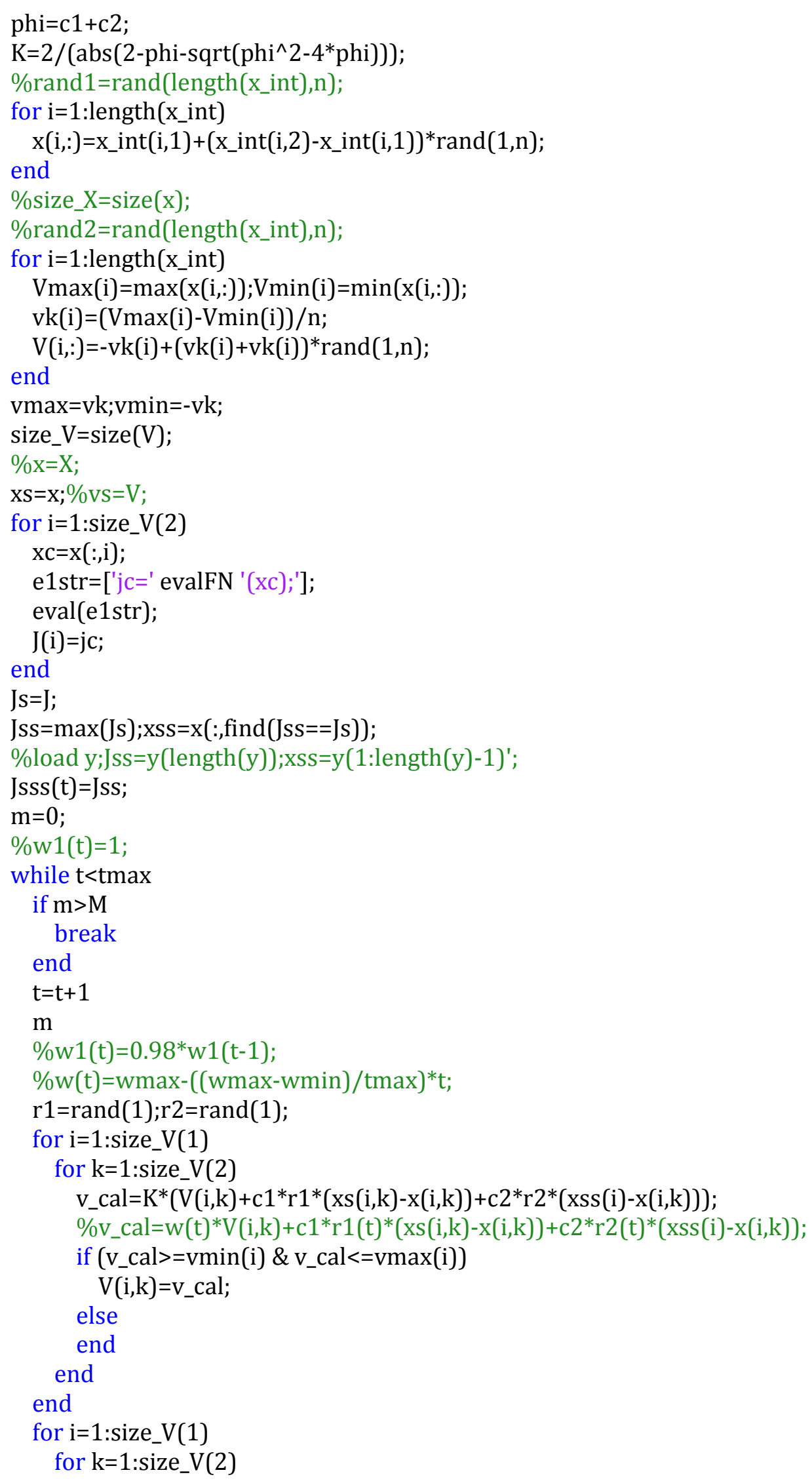




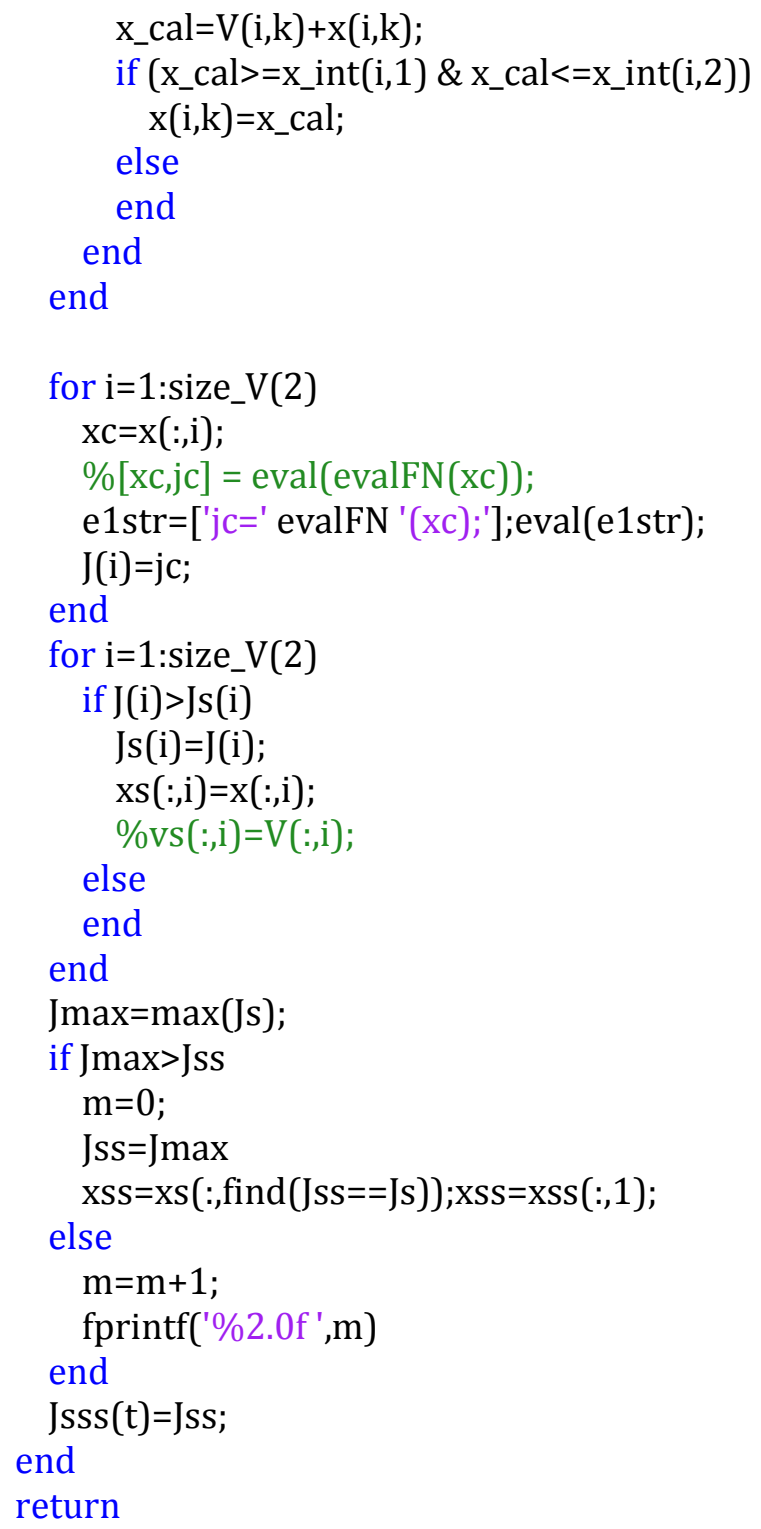




\section{Reinforcement Learning}

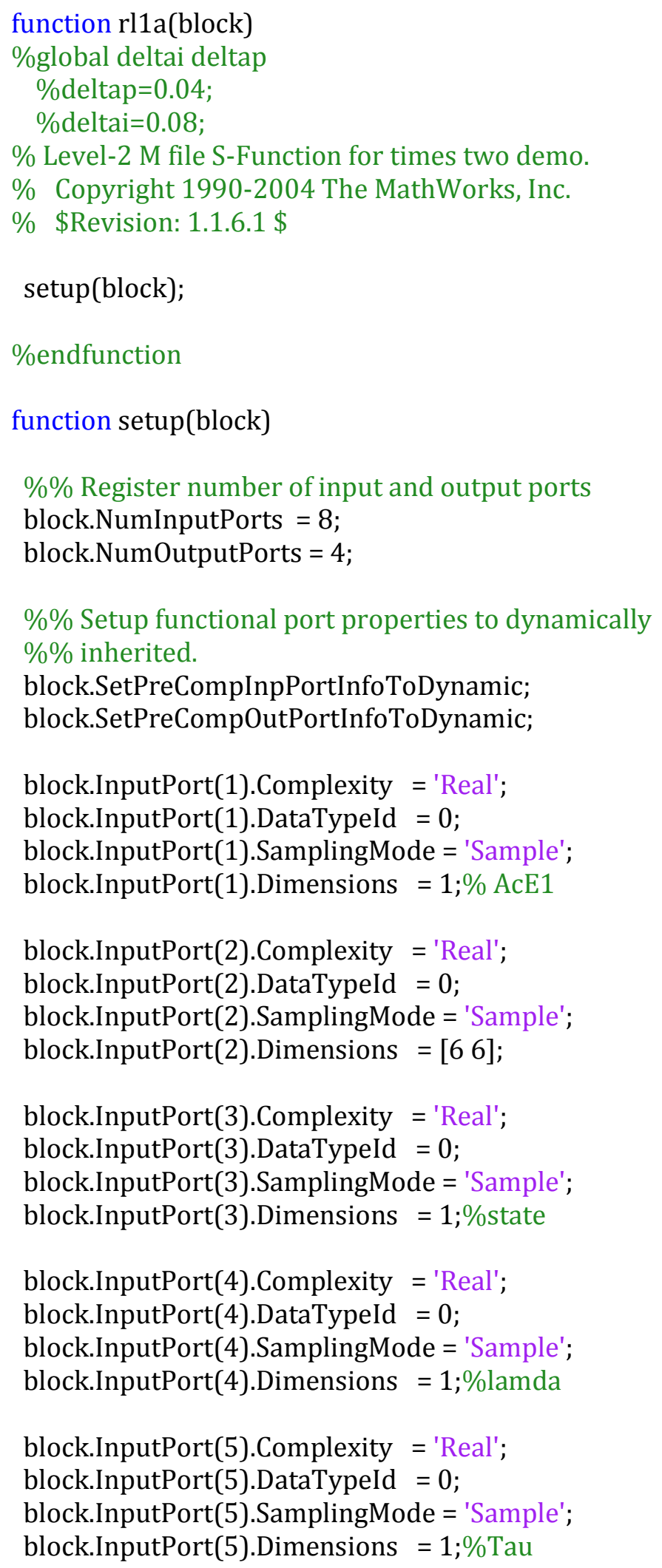




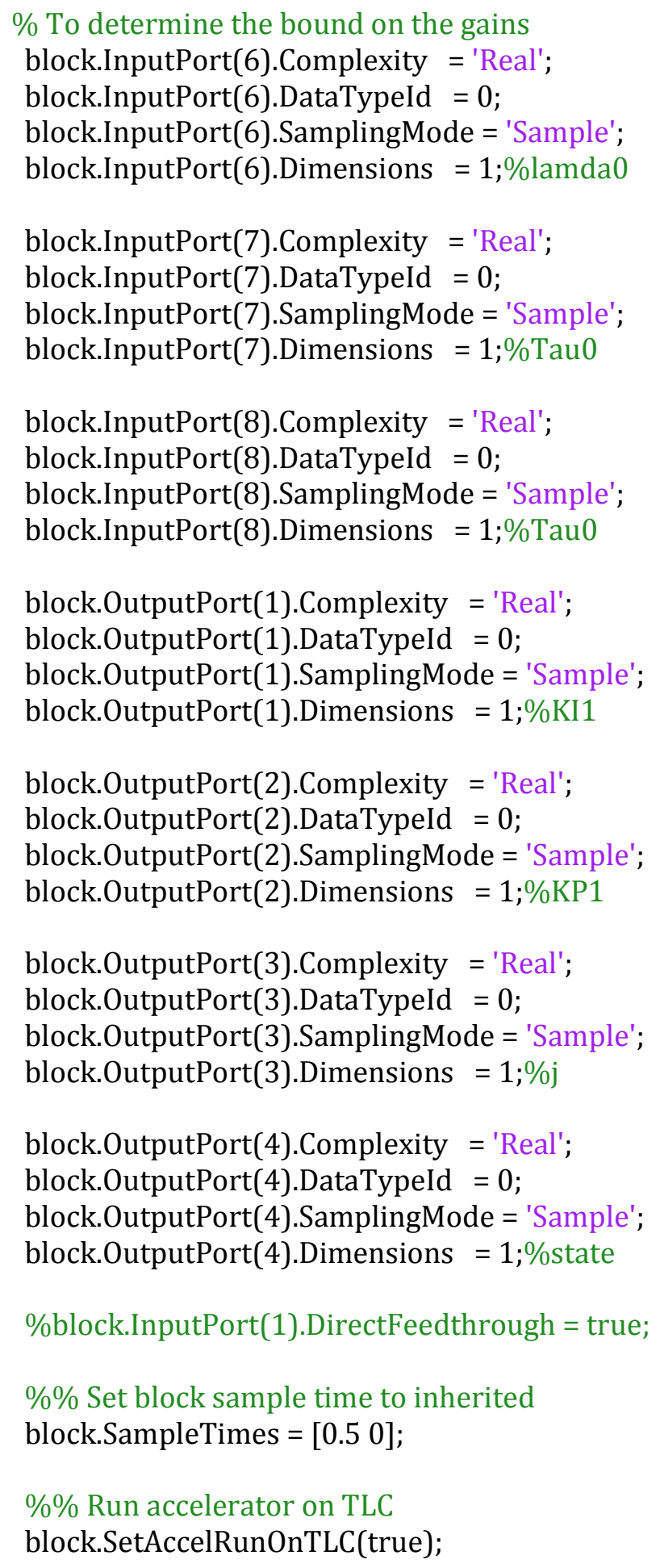




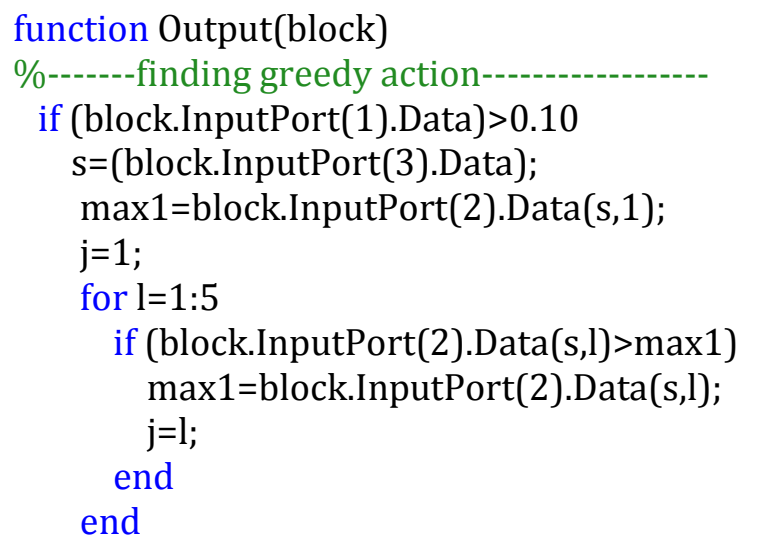

block.OutputPort(3).Data=j;

block.OutputPort(4).Data=block.InputPort(3).Data;

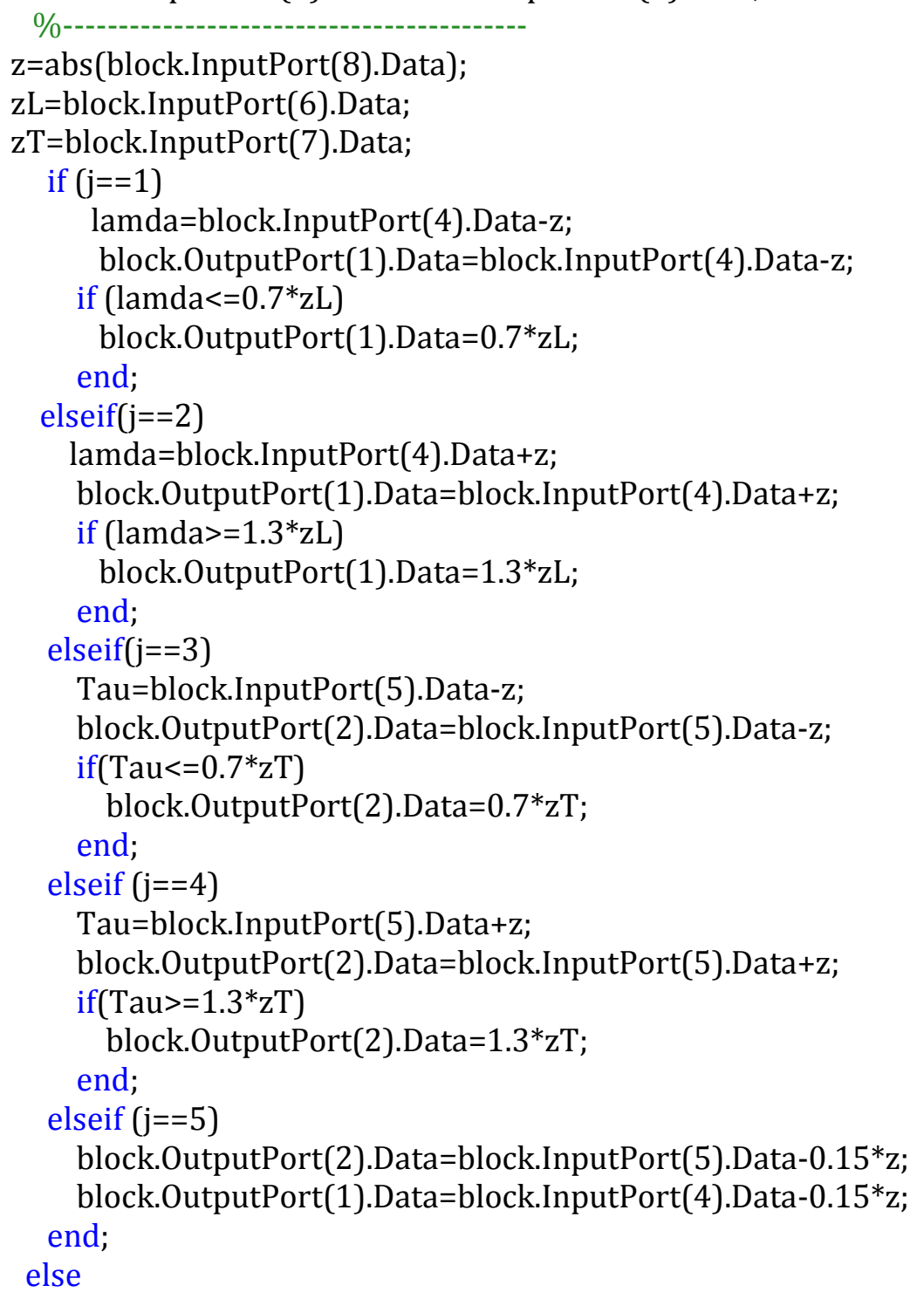

block.OutputPort(3).Data=6; 
block.OutputPort(4).Data=6;

block.OutputPort(1).Data=block.InputPort(4).Data;

block.OutputPort(2).Data=block.InputPort(5).Data;

end

\%endfunction

function rl2(block)

global al gama

$\mathrm{al}=0.9$;

gama $=0.9$;

\% Level-2 M file S-Function for times two demo.

\% Copyright 1990-2004 The MathWorks, Inc.

\% \$Revision: 1.1.6.1 \$

setup(block);

\%endfunction

function setup(block)

$\% \%$ Register number of input and output ports

block.NumInputPorts $=4$;

block.NumOutputPorts $=2$;

$\% \%$ Setup functional port properties to dynamically

$\% \%$ inherited.

block.SetPreCompInpPortInfoToDynamic;

block.SetPreCompOutPortInfoToDynamic;

block.InputPort(1).Complexity = 'Real';

block.InputPort(1).DataTypeId $=0$;

block.InputPort(1).SamplingMode = 'Sample';

block.InputPort(1).Dimensions = 1;\% PI

block.InputPort(2).Complexity = 'Real';

block.InputPort(2).DataTypeId $=0$;

block.InputPort(2).SamplingMode = 'Sample';

block.InputPort(2).Dimensions = [6 6];

block.InputPort(3).Complexity = 'Real';

block.InputPort(3).DataTypeId $=0$;

block.InputPort(3).SamplingMode = 'Sample';

block.InputPort(3).Dimensions $=1 ; \%$ state 


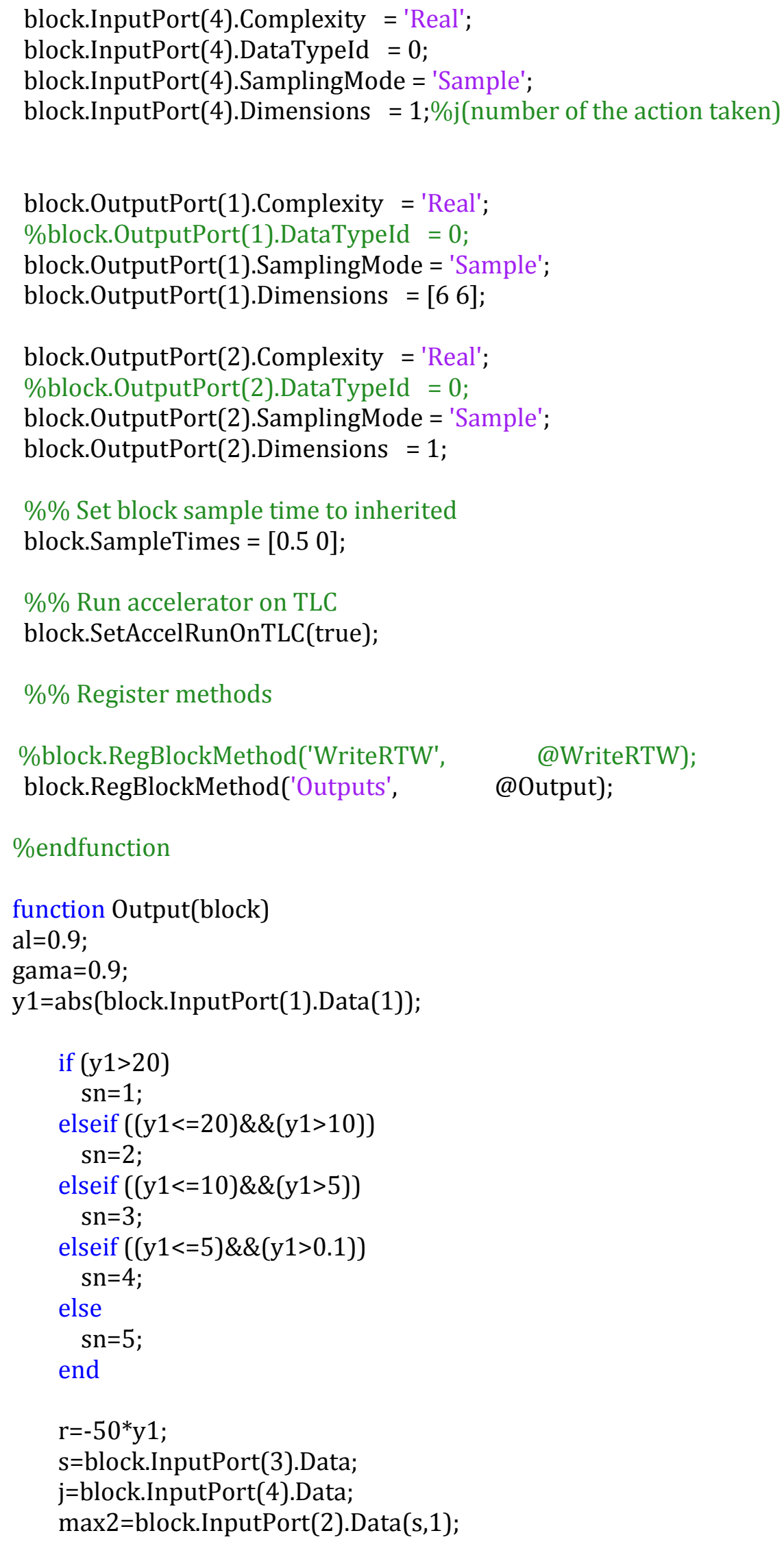




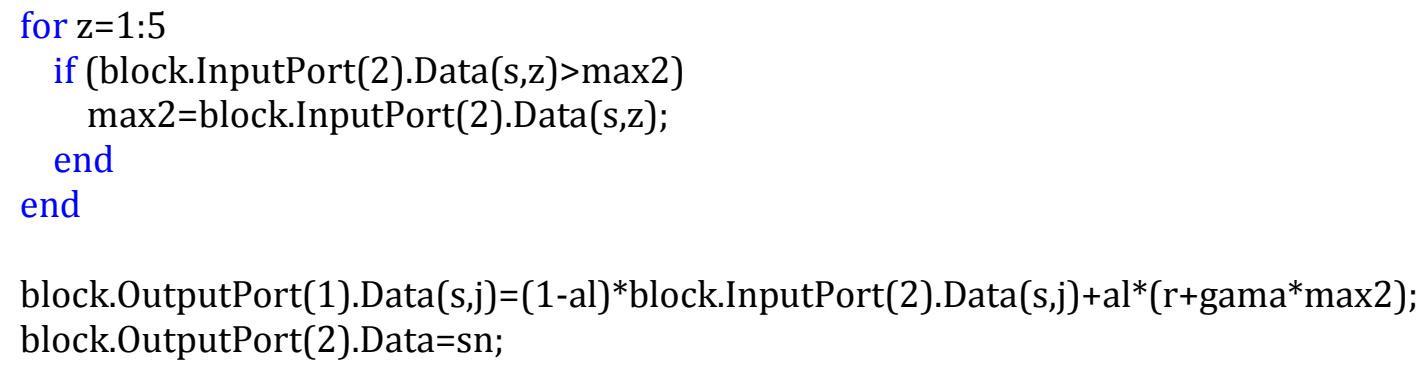

\%endfunction 


\section{REFERENCES}

[1] M. Amin, "Powering the 21st Century," IEEE Power \& Energy. March- April, 2005, pp. 93-96.

[2] IEEE/CIGRE Joint Task force on Stability Terms and Definitions, "Definition and Classification of Power System Stability," IEEE Transactions on Power Systems, Vol. 19, No. 2, pp. 1387-1401, 2004.

[3] P. Kundur, Power System Stability and Control, McGraw-Hill, 1994

[4] P. Kundur, "Effective Use of Power System Stabilizers for Enhancement of Power System reliability," PES, IEEE Power Engineering Society, Edmonton, Alta, canada, pp. 96-103, July 1999.

[5] Hingorani, N. G., “Flexible AC Transmission,” IEEE Spectrum, pp. 40-44, April 1993.

[6] Eberhart, R. C., and Kennedy J.,(1995) “ A New Optimizer Using Particle swarm Theory,” Proceedings of the Sixth International Symposium on Micro Machine and Human Science, Nagoya, Japan, 39-43. Piscataway, NJ: IEEE Service Center

[7] M. Glavic, Design of resistive brake controller for power system stability enhancement using reinforcement learning, IEEE Trans. Power Syst. 13 5, (September) (2005) 743-751.

[8] S. Eftekharnejad, and A Feliachi, Stability Enhancement Through Reinforcement Learning: Load Frequency Control Case Study, Bulk Power system Dynamics and Control, Charleston, S.C, USA, Aug. 19-24, 2007.

[9] K.Y. Lee, and M.A. El-Sharkawi, Modern Heuristic Optimization Techniques: Theory and Applications to Power Systems, Wiley- Interscience, 2008

[10] F. P. de Mello and C. Concordia, "Concepts of Synchronous Machine Stability as Affected by Excitation Control," IEEE Transaction on Power System, Vol. PAS-88 (4), pp. 316-329, 1969.

[11] G. E. Boukarim, S. Wang, J. H. Chow, G. N. Taranto, N. Martins, "A Comparison of Classical, Robust, and Decentralized Control designs for Multiple Power System Stabilizers," IEEE Transaction on Power Systems, Vol. 15, No. 4, November 2000.

[12] J. H. Chow, L. P. Harris, M. A. Kale, H. A. Othman, J. J. Sanchez-Gasca, G. E. Terwilliger, “ Robust control design of power system stabilizers using multivariable frequency domain techniques," Proceedings of the 29th IEEE Conference on Decision and Control, Honolulu, HI, USA, 1990

[13] Karimi, A. (2008), Power System Damping Controllers Design using a Backstepping Control Technique, (unpublished doctoral dissertation). West Virginia University, Morgantown, West Virginia

[14]S. J. Cheng, Y. S. Chow, O. P. Malik, G. S. Hope, “An Adaptive synchronous Machine Stabilizer,” IEEE Transactions on power Systems, Vol. 1, No. 3, pp. 101-109, 1986.

[15] A. L. B. do Bomfim, G. N. Taranto, D. M. Falcao, "Simultaneous Tuning of Power System Damping Controllers Using Genetic Algorithms," IEEE Transactions on Power System, Vol. 15, pp. 163-169, 2000.

[16] A. Hasanovic, A Feliachi, “Robust PSS Tuning Through Multiobjective Optimization,” PSCE, IEEE Power Systems And Exposition, New York, Oct, 2004.

[17] M. A. Abido, Y. L. Abdel-Magic, "Robust design of multimachine power system stabilizers using tabu search algorithm," IEE Proceedings Generation, Transmission and Distribution, Vol. 147, pp. 387-394, November 2000. 
[18] M. A. Abido, “Optimal Desing of Power System Stabilizers Using Particle Swarm Optimization," IEEE Transactions on Energy Conversion, Vol. 17, No. 3, September 2002.

[19] T. Yu, and W. Zhen, A Reinforcement Learning Approach to Power System Stabilizers, PES, IEEE Power and Energy Society General Meeting, Calgary, AB, July 26-30, 2009

[20] R. Hadidi, and B. Jeyasurya, Reinforcement learning approach for controlling power system stabilizers, Canadian Journal of Electrical and Computer Engineering 34, No 3 (2009) 99-103.

[21] Guo, Y., Hill, D. J., \& Wang, Y (2000), "Nonlinear decentralized control of large-scale power systems," Automatica, 36, $1275-1289$

[22] Zhu, C., Zhou, R, Wang, Y. “A New Decentralized Nonlinear Voltage Controller For Multimachine Power Systems,” IEEE Trans. On Power Systems, Vol 13, pp 211-216, February 1998.

[23] M. D. Ilic, H. Allen, W. Chapman, C. A. King, J. H. Lang, E. Litivinov, "Preventing future blackouts by means of enhanced electric power systems control: from complexity to order," Proceedings of the IEEE, Vol. 93, No 11, November 2005.

[24] Karimi, A., Feliachi, A., "Decentralized Extended-Backstepping Control of Power systems," IEEE PES General Meeting, 2006

[25] Karimi, S. Eftekharnejad, and A Feliachi, Reinforcement learning based backstepping control of power system oscillations, Int. J Elsevier on Electric Power Systems Research 79 (2009) 1511-1520.

[26] X. G. Yontag, S. K. Spurgeon, C. Edwards, “Decentralized Sliding Mode Control for Multimachine Power Systems Using Only Output Information," The $29^{\text {th }}$ Annual Conference of the IEEE on Industrial Electronics Society, IECON, Vol. 2, pp. 1944-1949, 2003.

[27] V. I. Utkin, "Variable Structure Control Systems with Sliding Modes," IEEE Transaction on Automatic Control, Vol. AC22, No. 2, pp. 210-222, 1977

[28] Edward, C., and Spurgeon, S., Sliding Mode Control: Theory and Applications, Taylor and Francis, London, 1998

[29] Nusawardhana, Zak, S. H, Crossley, W. A., "Nonlinear Synergetic Optimal Controllers," Journal of Guidance, Control and Dynamics, Vol. 30, No. 4, July-August 2007.

[30] Santi, E., Monti, A., Li, D., Proddutur, K., Dougal, R. A., " Synergetic Control for Power electronics Applications: a Comparison with the Sliding Mode Approach," Journals of Circuits, Systems and Computers, Vol. 13, No. 4, pp 737- 760, 2004.

[31] Bastos, J., Monti, A., Santi, E., "Design and implementation of a Nonlinear Speed Control for a PM Synchronous Motor Using The Synergetic Approach to Control Theory," Power Electronics Specialists Conference, 2004. PESC 04

[32] Kolesnikov, A., Veselov, G, Monti, A., Ponci, F, Santi, E, Dougal, R.,"'Synergetic Synthesis of DC- DC Boost Converter Controllers: Theory and Experimental Analysis," Applied Power Electronics Conference and Exposition, APEC 2002.

[33] Jiang, Z., "Design of Nonlinear Power System Stabilizer Using Synergetic Control Theory," IEEE Power Engineering Society General Meeting, 2007

[34] J. J. Sanchez-Gasca, "Coordination control of two FACTS devices for damping inter-area oscillations," IEEE PES summer meeting, Berlin, Germany, July 1997.

[35] P. Pourbiek, M. J. Gibbard, "Simultaneous coordination of Power System Stabilizers and FACTS device stabilizers in a multimachine power system for enhancing dynamic performance," IEEE Transaction on Power Systems, Vol. 13, No. 2, pp. 473-479, 1998

[36] Kolesnikov, A. A, Modern Applied control Theory: Synergetic Approach in Control Theory, TRTU, Moscow-Taganrog, 2000.

[37] Slotine, J., and Li, W., Applied Nonlinear Control, Prentice-Hall, Englewood Cliffs, NJ, 1991. 
[38] W.M. Haddad, V. Chellaboina, Nonlinear Dynamical Systems and Control: A Lyapunov-Based Approach, Princeton University Press 2008

[39] Sauer, P. W., Pai, M. A., “Power System Dynamics and Stability,” Prentice - Hall, Upper Saddle River, NJ, 1998

[40] Yan, R., Dong, Y., Saha, T. K., Majumdar, R., (2010) “A Power System Nonlinear Adaptive Decentralized Controller Design," Automatica, 46, 330-336.

[41] Krstic, M., Kanellakopoulos, L., and Kokotovic, P., Nonlinear and Adaptive Control Design, John Wiley \& Sons Inc., NY, 1995

[43] M. Clerc, J. Kennedy, “ The Particle Swarm-Explosion, Stability and Convergence in Multidimensional Complex Space,” IEEE Transactions on Evolutionary Computations, Vol. 6, No. 1, pp 58-73, 2002.

[44] B. Panigrahi, A. Abraham, and S. Das Eds., Computational Intelligence in Power Engineering.Berlin, Germany: SpringerVerlag, 2010.

[45] IEEE Recommended Practice for Excitation System Models for Power system Stability Studies, Energy Development and Power Generating Committee of the Power Engineering Society, 19, March 1992.

[46] Schoder, A., Hasanovic, A., and Feliachi, A., "PAT: A Power Analysis Toolbox for MATLAB/ Simulink," IEEE Trans. On Power Systems, Vol. 18, pp 42 -48, 2003

[47] Taoridi Ademoye, Ali Feliachi, “Decentralized Synergetic Control of Multimachine Systems” PSCE, IEEE Power Systems And Exposition, Phoenix, AZ, March 2011

[48] T. Ademoye, A. Feliachi, and A. Karimi, Coordination of Synergetic Excitation Controller and SVC-Damping Controller Using Particle Swarm Optimization, in: IEEE Power and Energy Society General Meeting, Detroit, MI, July 24- 29,2011

[49] M. Meissner, M. Schmuker, G. Schneider, Optimized particle swarm optimization (OPSO) and its application to artificial neural network training, BMC Bioinformatics 7 (125) (2006)

[50] D. Ernst, M. Glavic, and L Wehenkel, Power system stability control: reinforcement learning framework, IEEE Trans. Power Syst. 19 (February) (2004)

[51] A. Karimi, A. Feliachi, M. A. Choudhry, "On an SVC backstepping damping nonlinear controller design for power systems," iREP Symposium Bulk Power System Dynamics and Control- VII, Charleston, SC, 2007

[52] A. Fouad, V. Vittal, Power System Transient Stability Analysis Using Transient Energy Function Method, Kluwer Academic Publishers, 1989. 